\title{
Heterodimensional cycles, partial hyperbolicity and limit dynamics
}

\author{
by \\ L. J. Díaz (Rio de Janeiro) and J. Rocha (Porto) \\ To J. Palis on the occasion of his 60th birthday
}

\begin{abstract}
We study one-parameter families of diffeomorphisms unfolding heterodimensional cycles (i.e. cycles containing periodic points of different indices). We construct an open set of such arcs such that, for a subset of the parameter space with positive relative density at the bifurcation value, the resulting nonwandering set is the disjoint union of two hyperbolic basic sets of different indices and a strong partially hyperbolic set which is robustly transitive.

The dynamics of the diffeomorphisms we consider is partially hyperbolic with onedimensional central direction. The main tool for proving our results is the construction of a one-dimensional model given by an iterated function system which describes the limit dynamics in the central direction. For selected parameters of the arc, we translate properties of the model family to the diffeomorphisms.
\end{abstract}

\section{INTRODUCTION}

It is well known that there are two main mechanisms to destroy the stability of a system: loss of hyperbolicity of some periodic point and creation of a cycle. In this paper we focus our attention on the second one.

In dimension two, the creation of cycles is associated to homoclinic or heteroclinic tangencies, and all the hyperbolic periodic points involved in the cycle have the same index (equidimensional cycles). This kind of bifurcations is a well studied subject since the seventies; see, for instance, $\left[\mathrm{PT}_{2}\right]$ for an overview on this matter.

In higher dimensions there are cycles involving hyperbolic periodic points of different indices (heterodimensional cycles). The unfolding of this kind of cycles may lead to dynamical features completely different from the ones in

2000 Mathematics Subject Classification: 37C20, 37D30.

Research of L. J. Díaz partially supported by CNPQ, PRONEX-Dynamical Systems, and FAPERJ (Brazil), and Centro de Matemática da Universidade do Porto (Portugal).

Research of J. Rocha partially supported by FCT Sapiens 99-36581/99. 
the equidimensional case (for instance, the creation of robustly transitive sets of partially hyperbolic type). The study of some of these dynamical features is the goal of this paper.

Let us introduce some definitions and state the main result of this article.

Let $M$ be a compact, connected and boundaryless $n$-dimensional Riemannian manifold, $n \geq 3$, and denote by $\operatorname{Diff}^{\infty}(M)$ and $\mathcal{P}^{\infty}(M)$ the spaces of $C^{\infty}$-diffeomorphisms and of one-parameter families of diffeomorphisms of $M$, respectively, endowed with the usual $C^{1}$-topologies.

A diffeomorphism $f$ defined on $M$ exhibits a heterodimensional cycle associated to the hyperbolic periodic points $P$ and $Q$ if $W^{\mathrm{s}}(P)$ and $W^{\mathrm{u}}(Q)$ have a nontrivial transverse intersection, and $W^{\mathrm{u}}(P)$ and $W^{\mathrm{s}}(Q)$ have a quasi-transverse intersection (of codimension one) along the orbit of some point $X$. In this case, $\operatorname{index}(P)=\operatorname{index}(Q)+1$, where index $(R)$ denotes the dimension of the stable manifold of the hyperbolic periodic point $R$.

Given $f$ with a heterodimensional cycle as above we can consider compact parts $K^{\mathrm{s}}(Q)$ of $W^{\mathrm{s}}(Q)$ and $K^{\mathrm{u}}(P)$ of $W^{\mathrm{u}}(P)$ such that

$$
K^{\mathrm{s}}(Q) \cap K^{\mathrm{u}}(P)=\operatorname{int}\left(K^{\mathrm{s}}(Q)\right) \cap \operatorname{int}\left(K^{\mathrm{u}}(P)\right)=\left\{X_{f}\right\},
$$

where $X_{f}$ is a point of quasi-transverse intersection. Consider a $C^{1}$-neighbourhood $\mathcal{U}$ of $f$ such that the analytic continuations of $P, Q, K^{\mathrm{s}}(Q)$ and $K^{\mathrm{u}}(Q)$ are defined for all $g \in \mathcal{U}$ and let $\Gamma$ be the set of diffeomorphisms $g$ in $\mathcal{U}$ such that the continuations $K^{\mathrm{s}}\left(Q_{g}\right)$ and $K^{\mathrm{u}}\left(P_{g}\right)$ have an intersection as in (0.1). If $\mathcal{U}$ is small then $\Gamma$ is a codimension one submanifold of $\operatorname{Diff}^{\infty}(M)$ such that $\mathcal{U} \backslash \Gamma$ has two connected components, say $\mathcal{U}_{\Gamma}^{+}$and $\mathcal{U}_{\Gamma}^{-}$.

We consider arcs $\left(f_{t}\right)_{t \in[-1,1]}$ unfolding generically a heterodimensional cycle in $\Gamma$ at $t=0$, i.e., the curve $\left(f_{t}\right)_{t \in[-1,1]}$ is transverse to $\Gamma$ at $t=0$. Here we suppose that the cycles correspond to a first bifurcation, that is (for instance), the diffeomorphisms in the component $\mathcal{U}_{\Gamma}^{-}$are hyperbolic. Observe that associated to the submanifold $\Gamma$ there is an open set $\mathcal{U}_{\Gamma}$ in $\mathcal{P}^{\infty}(M)$ consisting of arcs unfolding generically cycles in $\Gamma$ (say at $t=0$, for simplicity). We assume that $f_{t} \in \mathcal{U}_{\Gamma}^{-}$for $t<0$ and $f_{t} \in \mathcal{U}_{\Gamma}^{+}$for $t>0$.

Given an arc $\left(f_{t}\right)_{t \in[-1,1]}$ our objective is to describe the dynamics of the diffeomorphisms $f_{t}$ in $\mathcal{U}_{\Gamma}^{+}$for a large set of parameters (a set of nonzero Lebesgue measure with positive relative density at the bifurcation $t=0$ ).

We consider a neighbourhood of the cycle, that is, an open set $\mathcal{V}$ containing all the elements of the cycle: the periodic points $Q$ and $P$, the intersection $W^{\mathrm{s}}(P) \cap W^{\mathrm{u}}(Q)$, and the orbit of the point $X_{f}$ of quasi-transverse intersection. One aims to describe the resulting nonwandering set, denoted by $\Omega\left(f_{t}\right)^{\prime}$, associated to the unfolding of the cycle, i.e., to characterize the dynamics of $\Omega\left(f_{t}\right)^{\prime}=\Omega\left(f_{t}\right) \cap \mathcal{V}$ for small $t>0$. This description can be very complicated, even in the case where the diffeomorphisms in the component $\mathcal{U}_{\Gamma}^{-}$are Morse-Smale or Axiom A. 
Let us introduce two sets of parameters:

- $H(s)$ is the set of parameters $t \in(0, s)$ such that $f_{t}$ is $\Omega$-stable and $\Omega\left(f_{t}\right)^{\prime}$ coincides with the (disjoint) union of the homoclinic classes of $P_{t}$ and $Q_{t}$ (the continuations of $P$ and $Q$ ).

- $B(s)$ is the set of parameters $t \in(0, s)$ such that the homoclinic classes associated to $P_{t}$ and to $Q_{t}$ have nonempty intersection.

Let us recall that the homoclinic class associated to a hyperbolic periodic point $R$ is the closure of the set $H(R)$ of transverse homoclinic points of $R$ (i.e., the transverse intersections between the invariant manifolds of $R$ ). Observe that, since $P_{t}$ and $Q_{t}$ have different indices, $f_{t}$ is nonhyperbolic (unstable) for every $t \in B(s)$.

In $\left[\mathrm{D}_{1}\right],\left[\mathrm{D}_{2}\right]$ and $\left[\mathrm{DR}_{1}\right]$ there are constructed codimension one submanifolds $\Gamma$ and open sets $\mathcal{U}_{\Gamma}$ as above such that $B(s)=(0, s)$ for every $\left(f_{t}\right)_{t \in[-1,1]} \in \mathcal{U}_{\Gamma}$ and small $s$. Moreover, the submanifold $\Gamma$ can be taken such that $\mathcal{U}_{\Gamma}^{-}$consists of Morse-Smale diffeomorphisms. We call this kind of cycles robustly nonhyperbolic (after the bifurcation).

On the other hand, in $\left[\mathrm{DR}_{2}\right]$ there is constructed a codimension one submanifold $\Upsilon$ such that every diffeomorphism in $\mathcal{U}_{\Upsilon}^{-}$is Morse-Smale and for every $\operatorname{arc}$ in $\mathcal{U}_{\Upsilon}$,

$$
\liminf _{t \rightarrow 0^{+}} \frac{|H(t)|}{t}>0 \text { and } \liminf _{t \rightarrow 0^{+}} \frac{|B(t)|}{t}>0,
$$

where $|A|$ denotes the Lebesgue measure of $A$. A natural question is if, in general, $H(t) \cup B(t)$ is equal to $[0, t]$ or, at least, has full relative density at the bifurcation. Our result gives a negative answer to these questions.

Clearly, the submanifolds $\Gamma$ and $\Upsilon$ above are disjoint. However, in both results the unstability (or nonhyperbolicity) is obtained by proving the existence of points in $W^{\mathrm{s}}\left(P_{t}\right) \cap W^{\mathrm{u}}\left(Q_{t}\right)$ belonging simultaneously to the homoclinic classes of $P_{t}$ and $Q_{t}$, which clearly prevents the hyperbolicity.

In $\left[\mathrm{D}_{1}\right],\left[\mathrm{D}_{2}\right]$ and $\left[\mathrm{DR}_{2}\right]$ the diffeomorphisms in $\Gamma$ or $\Upsilon$ are far from tangencies: it is not possible to create homoclinic tangencies associated to the periodic points $P$ and $Q$ by small perturbations. This condition implies some restrictions on the geometry of the intersections $W^{\mathrm{s}}(P) \cap W^{\mathrm{u}}(Q)$.

Actually, there is a much more general result. Consider a codimension one submanifold $\Gamma$ as above which is far from tangencies. Then for any arc in $\mathcal{U}_{\Gamma}$ unfolding generically a cycle, the set $B(s)$ has positive relative density at $t=0$ for all $s>0\left(\right.$ see $\left.\left[\mathrm{DR}_{3}\right]\right)$.

Summarizing, as far as we know, the reason of the nonhyperbolicity of the diffeomorphisms in $\mathcal{U}_{\Gamma}^{+}$is that there are nonwandering points in $W^{\mathrm{s}}\left(P_{t}\right) \cap$ $W^{\mathrm{u}}\left(Q_{t}\right)$ and the homoclinic classes of $P_{t}$ and $Q_{t}$ are not disjoint. 
Thus it is natural to ask whether the resulting nonwandering set is hyperbolic when both $\overline{H\left(P_{t}\right)}$ and $\overline{H\left(Q_{t}\right)}$ are hyperbolic (basic) sets, or when $W^{\mathrm{s}}\left(P_{t}\right) \cap W^{\mathrm{u}}\left(Q_{t}\right)$ consists of wandering points. Clearly, these two conditions are necessary for the hyperbolicity of $\Omega\left(f_{t}\right)^{\prime}$. In this paper we prove that they are not sufficient. For that we construct a codimension one submanifold $\Gamma$ of diffeomorphisms with heterodimensional cycles far from tangencies such that $\mathcal{U}_{\Gamma}^{-}$is contained in the Morse-Smale systems and, for every arc $\left(f_{t}\right)_{t \in[-1,1]}$ in $\mathcal{U}_{\Gamma}$, there exists a set of parameters $t$ with positive relative density at the bifurcation value such that:

- the homoclinic classes of $P_{t}$ and $Q_{t}$ are hyperbolic (basic) sets,

- every point of $W^{\mathrm{u}}\left(Q_{t}\right) \cap W^{\mathrm{s}}\left(Q_{t}\right)$ is wandering,

- the resulting nonwandering set is not hyperbolic, in fact, this set is strong partially hyperbolic, that is, the resulting nonwandering set has an $f_{t^{-}}$ invariant partially hyperbolic splitting of the form $E^{\mathrm{S}} \oplus E^{\mathrm{c}} \oplus E^{\mathrm{u}}$, where $E^{\mathrm{S}}$ is uniformly contracting, $E^{\mathrm{u}}$ is uniformly expanding, and $E^{\mathrm{c}}$ is nonhyperbolic and one-dimensional; see Section 9 for the precise definition.

Before stating our results more precisely, recall that a compact $f$-invariant set $\Lambda_{f}$ is robustly transitive if there are neighbourhoods $V$ of $\Lambda_{f}$ in the ambient manifold and $\mathcal{V}$ of $f$ in the space of $C^{1}$-diffeomorphisms such that the set

$$
\Lambda_{g}=\bigcap_{i \in \mathbb{Z}} g^{i}(\bar{V}) \subset V
$$

is transitive (i.e., the set is the closure of some orbit of it) for every $g \in \mathcal{V}$. For properties of robustly transitive sets we refer the reader to [DPU], [BDP] and $[\mathrm{BDPR}]$.

THEOREM. There exists a nonempty open set $\mathcal{A} \subset \mathcal{P}^{\infty}(M)$ such that for every arc $\left(f_{t}\right)_{t \in[-1,1]}$ in $\mathcal{A}$ :

(1) $\left(f_{t}\right)_{t \in[-1,1]}$ unfolds generically a heterodimensional cycle at $t=0$ associated to two hyperbolic fixed points $P_{0}$ and $Q_{0}$.

(2) There are sequences of pairwise disjoint intervals $\left[a_{k}, b_{k}\right]$ and of parameters $\left.c_{k} \in\right] a_{k}, b_{k}[$ such that

(i) for every parameter $t \in \bigcup_{k}\left[a_{k}, b_{k}\right]$ the homoclinic classes of $P_{t}$ and $Q_{t}$ are (hyperbolic) basic sets and there are a pair of hyperbolic periodic points $A_{t}$ and $R_{t}$ in the resulting nonwandering set of $f_{t}$ such that $A_{t}$ and $R_{t}$ do not belong to $\overline{H\left(P_{t}\right)} \cup \overline{H\left(Q_{t}\right)}$,

(ii) $f_{c_{k}}$ has a heterodimensional cycle associated to $A_{c_{k}}$ and $R_{c_{k}}$ and the arc unfolds generically such a cycle.

(3) For every $\left.t \in] c_{k}, b_{k}\right]$ the resulting nonwandering set of $f_{t}$ is hyperbolic and 


$$
\Omega\left(f_{t}\right)^{\prime}=\overline{H\left(P_{t}\right)} \cup \overline{H\left(Q_{t}\right)} \cup \mathcal{O}\left(A_{t}\right) \cup \mathcal{O}\left(R_{t}\right),
$$

where $\mathcal{O}(Y)$ denotes the orbit of $Y$.

(4) For every $t \in\left[a_{k}, c_{k}\right.$ [ the resulting nonwandering set of $f_{t}$ is the disjoint union of the homoclinic classes of $P_{t}$ and of $Q_{t}$ and a strong partially hyperbolic set $\Sigma_{t}$ which is robustly transitive and $\Sigma_{t}=\overline{H\left(A_{t}\right)}=\overline{H\left(R_{t}\right)}$.

(5) Let $\left.\left.H=\bigcup_{k}\right] c_{k}, b_{k}\right]$ and $S=\bigcup_{k}\left[a_{k}, c_{k}[\right.$. Then

$$
\liminf _{t \rightarrow 0} \frac{|H \cap[-t, t]|}{2 t}>0 \quad \text { and } \quad \liminf _{t \rightarrow 0} \frac{|S \cap[-t, t]|}{2 t}>0 .
$$

To prove this result we fix a special family $\left(f_{t}\right)_{t \in[-1,1]}$ of diffeomorphisms unfolding a heterodimensional cycle and begin by constructing a sequence of scaled intervals $\left[c_{k}, b_{k}\right]$ in the parameter space converging to $0^{+}$and such that, for every $\left.t \in] c_{k}, b_{k}\right]$, the resulting nonwandering set of $f_{t}$ is formed by the homoclinic classes of $P_{t}$ and $Q_{t}$, which are (disjoint) basic sets, and two new hyperbolic points $A_{t}$ and $R_{t}$ of indices equal to the indices of $P_{t}$ and $Q_{t}$, respectively. Moreover, for these parameters, $W^{\mathrm{u}}\left(R_{t}\right)$ and $W^{\mathrm{s}}\left(A_{t}\right)$ have a nonempty transverse intersection. Then we prove that for $t=c_{k}$ the invariant manifolds $W^{\mathrm{u}}\left(A_{t}\right)$ and $W^{\mathrm{s}}\left(R_{t}\right)$ have a quasi-transverse intersection, thus at $t=c_{k}$ there is a heterodimensional cycle associated to $A_{t}$ and $R_{t}$. This is done without destroying the hyperbolicity of $\overline{H\left(P_{t}\right)}$ and $\overline{H\left(Q_{t}\right)}$. The unfolding of each of these cycles leads to the creation of the (transitive) strong partially hyperbolic sets $\Sigma_{t}$ in the statement of the theorem.

For two-dimensional arcs bifurcating from Morse-Smale systems via homoclinic tangencies the set of parameters corresponding to independent saddles (i.e., with homoclinic classes trivial) has zero relative density at the bifurcation value. This follows from the fact that, with full relative measure at the bifurcation, the resulting nonwandering set is a nontrivial hyperbolic homoclinic class (the homoclinic class of the point involved in the tangency); see the constructions in $\left[\mathrm{PT}_{1}\right]$ and $\left[\mathrm{PT}_{2}\right]$. Items (3) and (5) of the theorem show that the situation in the heterodimensional context is quite different.

A minor modification of our construction shows the existence of open sets of arcs unfolding heterodimensional cycles such that, for a set of parameters having positive relative measure at the bifurcation parameter value, the resulting nonwandering set is equal to the disjoint union of a finite number of hyperbolic basic sets: the homoclinic classes of $P_{t}$ and $Q_{t}$ and an arbitrarily large number of independent saddles. As far as we know, it is an open question whether there coexist infinitely many independent saddles for heterodimensional cycles far from tangencies.

The dynamics after the unfolding of a heterodimensional cycle associated to $P$ and $Q$ (where the index of $P$ is less than the index of $Q$ ) depends strongly on the action of $f$ in $W^{\mathrm{s}}(P) \cap W^{\mathrm{u}}(Q)$, called central dynamics. In our case, this intersection is a curve which is diffeomorphic to an open 
interval $I$. What we do is to fix a one-dimensional map $\Pi: I \rightarrow I$ and construct the open set $\mathcal{A}$ mentioned in the theorem in such a way that the central dynamics of $\operatorname{arcs}$ in $\mathcal{A}$ is close to $\Pi$. The key idea of the construction is that we fix a model cycle, whose central dynamics is related to $\Pi$, and we prove that (up to an essentially affine reparametrization and a change of coordinates) the cycles at $c_{k}$ are exactly as the model one. In other words, we have a kind of renormalization and limit dynamics. This allows us to translate properties of the model (also in the sense of measure of parameters) to the initial family.

More precisely, in our construction the parameters $t$ corresponding to secondary cycles (i.e., parameters $t>0$ such that $f_{t}$ has a cycle associated to $P_{t}$ and $Q_{t}$ ) play a key role. We see that there is a sequence of parameters $t_{k} \rightarrow 0^{+}$corresponding to secondary cycles such that (up to the period) the central dynamics is essentially the same. In fact, the one-dimensional model $\Pi$ induces an iterated function system describing such a central dynamics. We construct an arc $\left(f_{t}\right)_{t \in[-1,1]}$ unfolding a heterodimensional cycle such that for parameters close to the $t_{k}$ the central dynamics is given by such an iterated function system, which is introduced in Section 3. In Sections 3.1 and 3.3 we describe the dynamics of this model. Using this model we deduce that the homoclinic classes of $P_{t}$ and $Q_{t}$ are both hyperbolic for parameters close to $t_{k}$ (see Section 7).

We see that the unfolding of the secondary cycles leads to the creation of two new saddles $A_{t}$ and $R_{t}$, with indices respectively equal to the ones of $P_{t}$ and $Q_{t}$. These new saddles are dynamically independent of the points $P_{t}$ and $Q_{t}$ (i.e., they are not homoclinically related to $P_{t}$ and $Q_{t}$ ). On the other hand, as the parameter changes new heterodimensional cycles (now associated to $A_{t}$ and $R_{t}$ ) are created. The unfolding of these cycles does not affect the dynamics of the homoclinic classes of $P_{t}$ and $Q_{t}$, which remains hyperbolic. To get the strong partially hyperbolic transitive sets $\Sigma_{t}$ in the theorem we analyze the unfolding of these new cycles associated to $A_{t}$ and $R_{t}$.

This analysis is done in the following way. We see that the model dynamics for the secondary cycle induces a new model dynamics for the unfolding of the cycle associated to $A_{t}$ and $R_{t}$. Using this model we prove that after the bifurcation the homoclinic classes of $A_{t}$ and $R_{t}$ are equal and form a robustly transitive set (in this case the cycle is unfolded as $t$ decreases). A model for the unfolding of primary heterodimensional cycles and the induced model for the cycles associated to $A_{t}$ and $R_{t}$ are presented in Sections 3.4 and 4.2 .

We end this introduction with two remarks about the phenomenon of creation and annihilation of periodic points and the topological entropy of the diffeomorphisms $f_{t}$. 
First observe that in the logistic family $\ell_{\lambda}(x)=\lambda-x^{2}$ (which is a model for understanding the dynamics of homoclinic bifurcations) there are only orbit creation parameters and no orbit annihilation parameters, meaning that periodic orbits are created monotonically as the parameter varies (see for instance $[\mathrm{MT}]$ ). In $[\mathrm{KKY}]$ an anti-monotonicity theorem is stated in the setting of homoclinic bifurcations: there is concurrent creation and annihilation of periodic points in the unfolding of a homoclinic tangency.

Observe that the families of diffeomorphisms in this paper exhibit the phenomenon of annihilation of robustly transitive sets: the robustly transitive sets $\Sigma_{t}$ are created and defined for all $t \in\left[a_{k}, c_{k}\right.$ [ and, as the parameter increases, disappear at $t=c_{k}$ and split into two hyperbolic periodic points. Using $\left[\mathrm{DR}_{3}\right.$, Theorem E], which says that there are intervals $E_{k}$ such that for every $t \in E_{k}$ the resulting nonwandering set $\Omega\left(f_{t}\right)^{\prime}$ is a robustly transitive set which is not hyperbolic, one gets immediately an anti-monotonicity theorem for robustly transitive sets:

Anti-Monotonicity Theorem (for robustly transitive sets). Consider $\left(f_{t}\right) \in \mathcal{A}, \mathcal{A}$ as in the Theorem. Then in any neighbourhood of $t=0$ (in the parameter space) there are infinitely many parameters corresponding to creation and to annihilation of robustly transitive sets.

The previous question leads naturally to the problem of the (monotonicity of the) variation of the entropy. For arcs of diffeomorphisms as considered here this question is open. Note that, since the homoclinic classes of $P_{t}$ and $Q_{t}$ are nontrivial for all $t>0$, the topological entropy $h\left(f_{t}\right)$ of $f_{t}$ is positive for all $t>0$. Observe that the arcs in $\left[\mathrm{DR}_{1}\right]$ exhibit anti-monotonicity of the entropy: in such a case the homoclinic classes of $P_{t}$ and $Q_{t}$ are completely destroyed for many parameters $t$, being simultaneously trivial, thus $h\left(f_{t}\right)=0$ for these parameters.

This paper is organized as follows. In Section 1 we describe a special family of diffeomorphisms unfolding a heterodimensional cycle.

In Section 2 we describe the typical trajectories of points in the resulting nonwandering set and introduce the families of iterated functions $G_{n, m}^{t}$ describing the central dynamics. The one-dimensional map $\Pi$ giving the central dynamics is introduced in Section 3. In Sections 3 and 4 we introduce the iterated function systems describing the unfolding of secondary cycles and primary cycles associated to $A_{t}$ and $R_{t}$ and study the dynamics of these systems.

In Section 5 we construct the sequence of scaled intervals $\left[a_{k}, b_{k}\right]$ referred to in the statement of the theorem and construct reparametrizations having uniformly bounded distortion. Moreover, for such intervals we get an accurate control of the central dynamics in terms of the map $\Pi$. 
In Section 6 we translate the properties of the model iterated function system to the true maps $G_{n, m}^{t}$. In Section 7 we prove that for any parameter $t$ in $\left[a_{k}, b_{k}\right]$ the homoclinic classes associated to $P_{t}$ and $Q_{t}$ are hyperbolic basic sets. For these intervals we also prove the existence of two independent saddles $A_{t}$ and $R_{t}$ of different indices.

In Section 8 we see that for parameters in $] c_{k}, b_{k}$ ] the resulting nonwandering set is the (disjoint) union of the homoclinic classes of $P_{t}$ and $Q_{t}$ and the orbit of the saddles $A_{t}$ and $R_{t}$. We also get generic cycles associated to these saddles for the parameters $c_{k}$.

Finally, in Section 9, using such cycles, we get the transitive strong partially hyperbolic sets $\Sigma_{t}=\overline{H\left(R_{t}\right)}=\overline{H\left(A_{t}\right)}$ for all $t$ in the interval $\left[a_{k}, c_{k}[\right.$.

Acknowledgements. The authors thank the referee for useful comments which, in particular, contributed to improving the presentation of the paper.

\section{UNFOLDING HETERODIMENSIONAL CYCLES}

1.1. Heterodimensional cycles. We begin by considering a $C^{\infty}$-diffeomorphism $f: \mathbb{R}^{n} \rightarrow \mathbb{R}^{n}$ having two hyperbolic fixed points, $P$ and $Q$, satisfying the following conditions:

(C1) The stable bundles of $P$ and of $Q$ have dimensions $r+1$ and $r$, respectively, i.e., the points $P$ and $Q$ have indices $r+1$ and $r$.

(C2) $\quad W^{\mathrm{u}}(Q) \cap W^{\mathrm{s}}(P)=W^{\mathrm{u}}(Q) \pitchfork W^{\mathrm{s}}(P)=\gamma$, where $\gamma$ is an $f$-invariant curve with endpoints $P$ and $Q$.

(C3) There exist $N_{2} \in \mathbb{N}$ and a point $X_{0}$ such that

- $f^{N_{2}}\left(W_{\mathrm{loc}}^{\mathrm{u}}(P)\right) \cap W_{\mathrm{loc}}^{\mathrm{s}}(Q)=\left\{X_{0}\right\}$,

- $\operatorname{dim}\left(T_{X_{0}} W^{\mathrm{u}}(P)+T_{X_{0}} W^{\mathrm{s}}(Q)\right)=n-1$,

- $W^{\mathrm{u}}(P) \cap W^{\mathrm{s}}(Q)=\bigcup_{n \in \mathbb{Z}} f^{n}\left(X_{0}\right)$,

i.e., the invariant manifolds $W^{\mathrm{s}}(P)$ and $W^{\mathrm{u}}(Q)$ are quasi-transverse at the heteroclinic point $X_{0}$.

(C4) There are compact neighbourhoods of $P$ and $Q$, say $\mathcal{U}_{P}$ and $\mathcal{U}_{Q}$, such that $\left.f\right|_{\mathcal{U}_{P}}$ and $\left.f\right|_{\mathcal{U}_{Q}}$ are linear (or $C^{1}$-linearizable).

(C5) The derivative of $f$ at $P, D f(P)$, (resp. at $Q, D f(Q)$ ) has a positive contracting (resp. expanding) eigenvalue of multiplicity one, $\lambda$ (resp. $\beta$ ), such that $|\sigma|<\lambda<1$ (resp. $1<\beta<|\sigma|$ ) for every contracting (resp. expanding) eigenvalue $\sigma$ of $D f(P)$ (resp. of $D f(Q)$ ) different from $\lambda$ (resp. $\beta$ ).

(C6) $\gamma \cap \mathcal{U}_{P}$ (resp. $\gamma \cap \mathcal{U}_{Q}$ ) is contained in the eigenspace associated to $\lambda$ (resp. $\beta$ ).

(C7) Hyperbolicity of the positive limit set at the bifurcation: the diffeomorphism $f$ is at the boundary of the Morse-Smale systems and its 
positive limit set, $L^{+}(f)$, is hyperbolic and the points $P$ and $Q$ are isolated in $L^{+}(f)$.

Let us make some comments about the conditions above:

Conditions (C1)-(C3) mean that the diffeomorphism $f$ has a heterodimensional cycle associated to the points $P$ and $Q$ (see Figure 1).

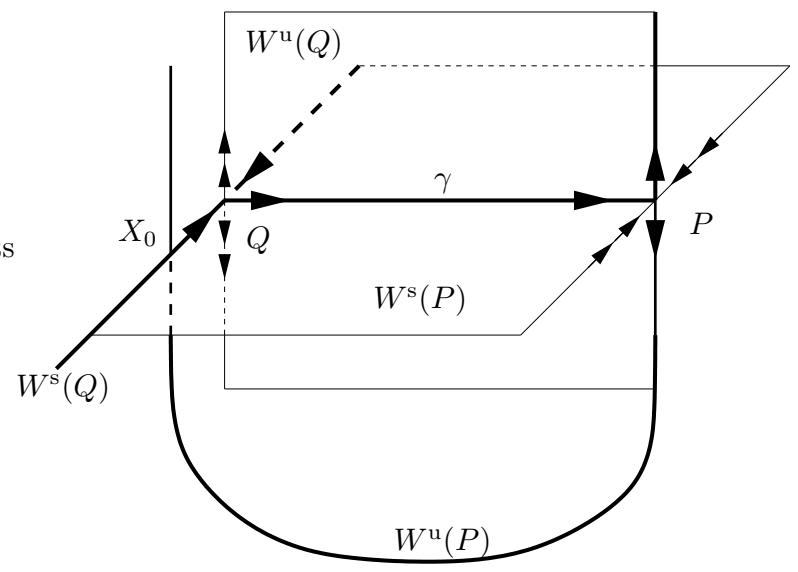

Fig. 1. A heterodimensional cycle

Condition (C4) is a technical assumption and can be guaranteed by imposing a finite number of nonresonance (open) conditions on the eigenvalues of $D f(P)$ and of $D f(Q)$.

Condition (C5) is a necessary condition for the cycle associated to $f$ to be a first bifurcation (see the arguments in $\left[\mathrm{DR}_{2}\right.$, Section 3]).

Condition (C6) is assured when the curve $\gamma$ is transverse to both the strong stable and the strong unstable foliations of $P$ and $Q$ (see definitions in Section 1.2 below). This condition means that $\gamma$ is simultaneously a separatrix of a centre stable manifold of $P$ and of a centre unstable manifold of $Q$.

Condition (C7) allows us to localize the nonwandering set after the unfolding of the cycle and implies that there is a spectral decomposition $\left\{\Lambda_{i}\right\}_{i=1}^{d}$ of $L^{+}(f)$ with $P=\Lambda_{1}$ and $Q=\Lambda_{2}$ (see $\left.\left[\mathrm{N}_{1}\right]\right)$. Write

$$
\Lambda=\left(\bigcup_{i \in \mathbb{Z}} f^{i}\left(X_{0}\right)\right) \cup\{P, Q\} \cup \gamma
$$

Given $i \in\{3, \ldots, d\}$ we say that $\Lambda_{i}>\Lambda$ (resp. $\Lambda_{i}<\Lambda$ ) if there exists a sequence $i_{1}, \ldots, i_{m}$, with $i_{1} \in\{1,2\}$ and $i_{m}=i$, such that $W^{\mathrm{u}}\left(\Lambda_{i_{j+1}}\right) \cap$ $W^{\mathrm{s}}\left(\Lambda_{i_{j}}\right) \neq \emptyset\left(\right.$ resp. $\left.W^{\mathrm{s}}\left(\Lambda_{i_{j+1}}\right) \cap W^{\mathrm{u}}\left(\Lambda_{i_{j}}\right) \neq \emptyset\right)$ for every $j \in\{1, \ldots, m-1\}$. Let

$$
\Lambda_{-}=\bigcup_{\Lambda_{i}<\Lambda} \Lambda_{i} \text { and } \Lambda_{+}=\bigcup_{\Lambda_{i}>\Lambda} \Lambda_{i} .
$$


By $\left[\mathrm{N}_{1}\right]$ there exists a filtration associated to $L^{+}(f)$, that is, there are compact sets $M_{1}$ and $M_{2}, M_{1} \subset M_{2}$, such that

$$
\begin{gathered}
\Lambda_{-} \subset \operatorname{int}\left(M_{1}\right), \quad \Lambda_{+} \subset M \backslash M_{2}, \quad \Lambda \subset M_{2} \backslash M_{1}, \\
f\left(M_{1}\right) \subset \operatorname{int}\left(M_{1}\right), \quad f\left(M_{2}\right) \subset \operatorname{int}\left(M_{2}\right) .
\end{gathered}
$$

In what follows we assume that the neighbourhoods $\mathcal{U}_{P}$ and $\mathcal{U}_{Q}$ in $(\mathrm{C} 4)$ and the local invariant manifolds of $P$ and $Q$ are chosen such that

$$
W_{\text {loc }}^{i}(R)=\mathcal{C C}\left(W^{i}(R) \cap \mathcal{U}_{R}, R\right), \quad i=\mathrm{s} \text { or } \mathrm{u} \text { and } R=P \text { or } Q,
$$

where $\mathcal{C C}(A, Z)$ denotes the connected component of the set $A$ that contains the point $Z$.

1.2. Product structure of the cycle. We assume that in our local linearizing coordinates in $\mathcal{U}_{P}$ and $\mathcal{U}_{Q}$, say $\left(x^{\mathrm{s}}, x^{\mathrm{c}}, x^{\mathrm{u}}\right), x^{\mathrm{s}} \in \mathbb{R}^{r}, x^{\mathrm{c}} \in \mathbb{R}$, and $x^{\mathrm{u}} \in \mathbb{R}^{n-r-1}$, we have $P=\left(0^{\mathrm{s}}, 0,0^{\mathrm{u}}\right)$ and $Q=\left(0^{\mathrm{s}}, 0,0^{\mathrm{u}}\right)$. Moreover,

$$
\begin{array}{ll}
W_{\text {loc }}^{\mathrm{s}}(P)=\left\{x^{\mathrm{u}}=0^{\mathrm{u}}\right\}, & W_{\mathrm{loc}}^{\mathrm{u}}(P)=\left\{\left(x^{\mathrm{s}}, x^{\mathrm{c}}\right)=\left(0^{\mathrm{s}}, 0\right)\right\}, \\
W_{\mathrm{loc}}^{\mathrm{s}}(Q)=\left\{\left(x^{\mathrm{c}}, x^{\mathrm{u}}\right)=\left(0,0^{\mathrm{u}}\right)\right\}, & W_{\mathrm{loc}}^{\mathrm{u}}(Q)=\left\{x^{\mathrm{s}}=0^{\mathrm{s}}\right\}, \\
W_{\mathrm{loc}}^{\mathrm{ss}}(P)=\left\{\left(x^{\mathrm{c}}, x^{\mathrm{u}}\right)=\left(0,0^{\mathrm{u}}\right)\right\}, & W_{\mathrm{loc}}^{\mathrm{uu}}(P)=\left\{\left(x^{\mathrm{c}}, x^{\mathrm{s}}\right)=\left(0,0^{\mathrm{s}}\right)\right\} .
\end{array}
$$

In this way the leaves of the local strong stable, strong unstable and central foliations in $\mathcal{U}_{P}$ and $\mathcal{U}_{Q}$ are parallel to $\mathbb{R}^{r} \times\left\{\left(0,0^{\mathrm{u}}\right)\right\},\left\{\left(0^{\mathrm{s}}, 0^{\mathrm{u}}\right)\right\} \times$ $\mathbb{R}^{n-r-1}$, and $\left\{0^{\mathrm{s}}\right\} \times \mathbb{R} \times\left\{0^{\mathrm{u}}\right\}$, respectively. We denote these foliations by $\mathcal{F}^{\mathrm{s}}$, $\mathcal{F}^{\mathrm{u}}$ and $\mathcal{F}^{\mathrm{c}}$, respectively. The leaf of $\mathcal{F}^{j}$ containing $X$ is denoted by $F^{j}(X)$.

Let $I$ be a fundamental domain of $\left.f\right|_{\gamma \cap \mathcal{U}_{Q}}$ and $N_{1}$ the first natural number such that $f^{N_{1}}(I)=J$ is contained in the interior of $\mathcal{U}_{P}$ (we call $N_{1}$ the

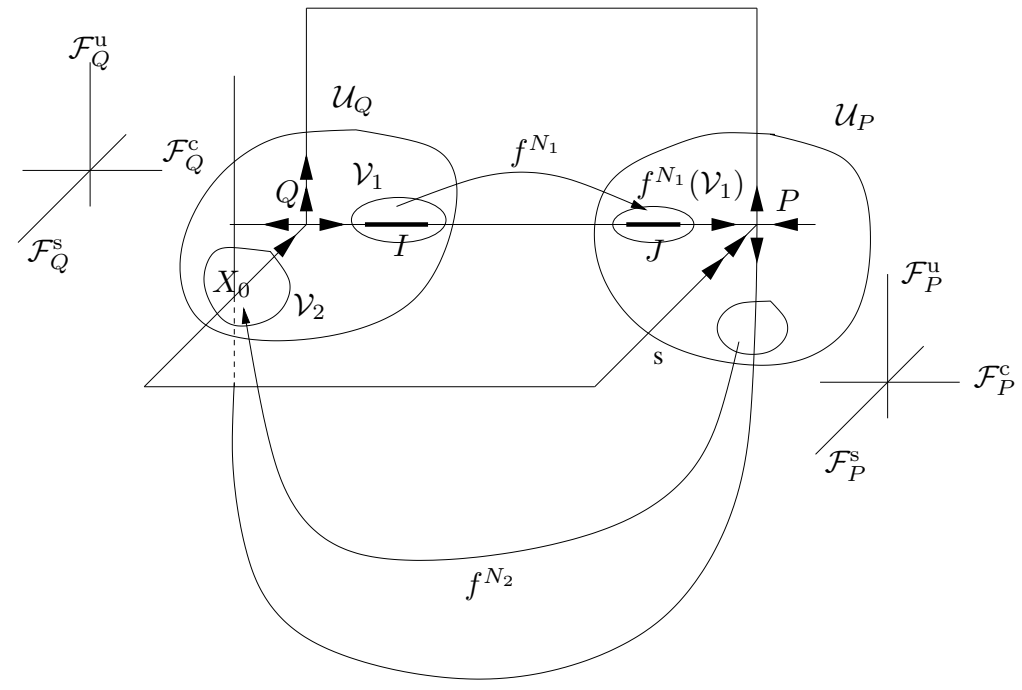

Fig. 2. Elements of the cycle 
transition time from $\mathcal{U}_{Q}$ to $\mathcal{U}_{P}$ ). Observe that we can (and do) assume that the heteroclinic point $X_{0}$ and the natural number $N_{2}$ in (C3) are such that $f^{-N_{2}}\left(X_{0}\right) \in \operatorname{int}\left(\mathcal{U}_{P}\right)$ and $f^{-i}\left(X_{0}\right) \notin \mathcal{U}_{P}$ for all $i=0,1, \ldots, N_{2}-1$. We say that $N_{2}$ is the transition time from $\mathcal{U}_{P}$ to $\mathcal{U}_{Q}$.

Consider now neighbourhoods $\mathcal{V}_{1}$ of $I$ and $\mathcal{V}_{2}$ of $X_{0}$ such that $f^{N_{1}}\left(\mathcal{V}_{1}\right)$ is contained in the interior of $\mathcal{U}_{P}, \mathcal{V}_{2}$ is contained in the interior of $\mathcal{U}_{Q}$ and disjoint from $f\left(\mathcal{V}_{2}\right), f^{-N_{2}}\left(\mathcal{V}_{2}\right)$ is contained in $\mathcal{U}_{P}$, and $f^{-j}\left(\mathcal{V}_{2}\right) \cap\left(\mathcal{U}_{Q} \cup \mathcal{U}_{P}\right)$ $=\emptyset$ for all $1 \leq j \leq N_{2}$.

Assume that for all $Z$ in $\mathcal{V}_{1}$ or $\mathcal{V}_{2}$ and every $i \in\{\mathrm{s}, \mathrm{c}, \mathrm{u}\}$ one has:

(F1) $f$-invariance of the foliations:

If $Z \in \mathcal{V}_{1}$ then $\mathcal{C C}\left(f^{N_{1}}\left(F^{i}(Z)\right) \cap \mathcal{U}_{P}, f^{N_{1}}(Z)\right) \subseteq F^{i}\left(f^{N_{1}}(Z)\right)$.

If $Z \in f^{-N_{2}}\left(\mathcal{V}_{2}\right)$ then $\mathcal{C C}\left(f^{N_{2}}\left(F^{i}(Z)\right) \cap \mathcal{U}_{Q}, f^{N_{2}}(Z)\right) \subseteq F^{i}\left(f^{N_{2}}(Z)\right)$.

Condition (F1) assures the $f$-invariance of the foliations along the cycle (the transition maps $f^{N_{1}}$ from $\mathcal{U}_{Q}$ to $\mathcal{U}_{P}$ and $f^{N_{2}}$ from $\mathcal{U}_{P}$ to $\mathcal{U}_{Q}$ keep invariant the stable, unstable, and central foliations).

Using the $f$-invariance of the foliations $\mathcal{F}^{\mathrm{s}}, \mathcal{F}^{\mathrm{u}}$ and $\mathcal{F}^{\mathrm{u}}$ we extend these foliations to the open set $\mathcal{V}$ given by

$$
\mathcal{V}=\mathcal{U}_{P} \cup \mathcal{U}_{Q} \cup\left(\bigcup_{i=1}^{N_{1}-1} f^{i}\left(\mathcal{V}_{1}\right)\right) \cup\left(\bigcup_{i=1}^{N_{2}-1} f^{-i}\left(\mathcal{V}_{2}\right)\right) .
$$

In this way we get $f$-invariant foliations defined in the whole $\mathcal{V}$ that, for notational simplicity, we also denote by $\mathcal{F}^{\mathrm{s}}, \mathcal{F}^{\mathrm{u}}$ and $\mathcal{F}^{\mathrm{c}}$.

We now consider holonomies and projections along the leaves of the stable and unstable foliations above. First, using our local coordinates, we define the local centre-stable and centre-unstable manifolds of $Q$ and $P$ by

$$
\begin{aligned}
& W_{\mathrm{loc}}^{\mathrm{cs}}(Q)=\left\{X \in \mathcal{U}_{Q}: X=\left(x^{\mathrm{s}}, x^{\mathrm{c}}, 0^{\mathrm{u}}\right)\right\}, \\
& W_{\text {loc }}^{\mathrm{cu}}(P)=\left\{X \in \mathcal{U}_{P}: X=\left(0^{\mathrm{s}}, x^{\mathrm{c}}, x^{\mathrm{u}}\right)\right\} .
\end{aligned}
$$

Observe that in these coordinates we have $W_{\mathrm{loc}}^{\mathrm{s}}(P)=\left\{\left(x^{\mathrm{s}}, x^{\mathrm{c}}, 0^{\mathrm{u}}\right)\right\}$ and $W_{\mathrm{loc}}^{\mathrm{u}}(Q)=\left\{\left(0^{\mathrm{s}}, x^{\mathrm{c}}, x^{\mathrm{u}}\right)\right\}$.

Let

$$
W_{N_{2}}^{\mathrm{cs}}(Q)=f^{-N_{2}}\left(W_{\mathrm{loc}}^{\mathrm{cs}}(Q)\right), \quad W_{N_{2}}^{\mathrm{cu}}(P)=f^{N_{2}}\left(W_{\mathrm{loc}}^{\mathrm{cu}}(P)\right) .
$$

By shrinking the size of the local manifold $W_{\text {loc }}^{\mathrm{s}}(P)$ and recalling condition (C3), we can assume that for each point $X \in W_{\text {loc }}^{\mathrm{s}}(P)$ the leaf $F^{\mathrm{u}}(X)$ intersects $W_{N_{2}}^{\text {cs }}(Q)$ transversely just in one point. Analogously, we can assume that for each $X \in W_{\text {loc }}^{\mathrm{u}}(Q)$ the leaf $F^{\mathrm{s}}(X)$ intersects $W_{N_{2}}^{\mathrm{cu}}(P)$ transversely just in one point. We now define the following holonomies associated to these foliations:

$$
\begin{aligned}
& \pi^{\mathrm{u}}: W_{\mathrm{loc}}^{\mathrm{s}}(P) \rightarrow W_{N_{2}}^{\mathrm{cs}}(Q), \quad Z \mapsto \pi^{\mathrm{u}}(Z)=F^{\mathrm{u}}(Z) \cap W_{N_{2}}^{\mathrm{cs}}(Q), \\
& \pi^{\mathrm{s}}: W_{\mathrm{loc}}^{\mathrm{u}}(Q) \rightarrow W_{N_{2}}^{\mathrm{cu}}(P), \quad Z \mapsto \pi^{\mathrm{s}}(Z)=F^{\mathrm{s}}(Z) \cap W_{N_{2}}^{\mathrm{cu}}(P) .
\end{aligned}
$$




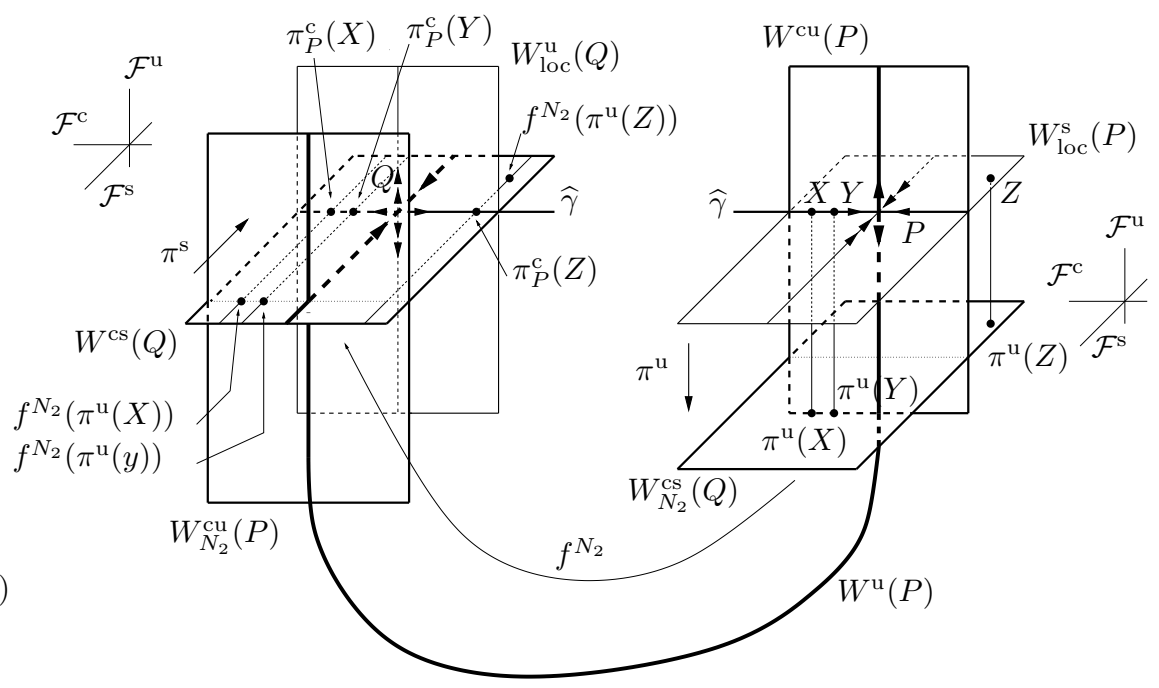

Fig. 3. Local manifolds and projections

Consider the curve

$$
\widehat{\gamma}=\gamma \cup\left\{Z=\left(0^{\mathrm{s}}, z, 0^{\mathrm{u}}\right): Z \in \mathcal{U}_{P}\right\} \cup\left\{Z=\left(0^{\mathrm{s}}, z, 0^{\mathrm{u}}\right): Z \in \mathcal{U}_{Q}\right\}
$$

and the projections $\widetilde{\pi}^{\mathrm{s}}$ and $\widetilde{\pi}^{\mathrm{u}}$ from $W_{\mathrm{loc}}^{\mathrm{cs}}(Q)$ to $\widehat{\gamma}$ and from $W_{\text {loc }}^{\mathrm{cu}}(P)$ to $\widehat{\gamma}$ along the leaves of the stable and the unstable foliations, respectively. Associated to these holonomies we define projections in the central direction as follows: $\pi_{P}^{\mathrm{c}}=\widetilde{\pi}_{Q}^{\mathrm{s}} \circ f^{N_{2}} \circ \pi^{\mathrm{u}}: W_{\mathrm{loc}}^{\mathrm{s}}(P) \rightarrow \widehat{\gamma}, \quad \pi_{Q}^{\mathrm{c}}=\widetilde{\pi}_{P}^{\mathrm{u}} \circ f^{-N_{2}} \circ \pi^{\mathrm{s}}: W_{\mathrm{loc}}^{\mathrm{u}}(Q) \rightarrow \widehat{\gamma}$.

We assume

Isometry and order preserving conditions:

- the maps $f^{N_{2}} \circ \pi^{\mathrm{u}}$ and $f^{-N_{2}} \circ \pi^{\mathrm{s}}$ are isometries,

- the restrictions to $\widehat{\gamma}$ of $\pi_{Q}^{\mathrm{c}}$ and of $\pi_{P}^{\mathrm{c}}$ are isometries and preserve the natural ordering $<$ of $\gamma$ (i.e., given $X, Y \in \widehat{\gamma}$ we write $X<Y$ if the distance from $P$ to $X$ along $\widehat{\gamma}$ is less than the distance from $P$ to $Y$ along $\widehat{\gamma})$.

Note that if the cycle corresponds to a first bifurcation then the projections above necessarily preserve the natural ordering in $\gamma\left(\right.$ see $\left.\left[\mathrm{DR}_{2}\right]\right)$. On the other hand, as in the case of condition (F1), condition (F2) does not hold in general, but in the general setting these projections can be viewed as quasi-isometries (see $\left[\mathrm{DR}_{3}\right.$, Section 6.2]).

\subsection{A model for the unfolding of the heterodimensional cy-} cle. In order to get a one-parameter family of diffeomorphisms unfolding the heterodimensional cycle of $f$ associated to $P$ and $Q$ above, we consider the arc $\left(f_{t}\right)_{t \in\left[-t_{0}, t_{0}\right]}$ defined by $f_{t}=\Phi_{t} \circ f$, where 
- $\Phi_{0}$ is the identity and, for each $t, \Phi_{t}$ is a diffeomorphism with support contained in a small neighbourhood $\mathcal{W}_{2}$ of $\mathcal{V}_{2}$ contained in $\mathcal{U}_{Q}$ such that $\mathcal{W}_{2} \cap f\left(\mathcal{W}_{2}\right)=\emptyset$

- in the coordinates $\left(x^{\mathrm{s}}, x^{\mathrm{c}}, x^{\mathrm{u}}\right)$, one has $\Phi_{t}\left(x^{\mathrm{s}}, x^{\mathrm{c}}, x^{\mathrm{u}}\right)=\left(x^{\mathrm{s}}, \phi_{t}\left(x^{\mathrm{c}}\right), x^{\mathrm{u}}\right)$ with $\phi_{t}\left(x^{\mathrm{c}}\right)=x^{\mathrm{c}}+t$ if $\left(x^{\mathrm{s}}, x^{\mathrm{c}}, x^{\mathrm{u}}\right) \in \mathcal{V}_{2}$, and $\phi_{t}\left(x^{\mathrm{c}}\right)=x^{\mathrm{c}}$ if $\left(x^{\mathrm{s}}, x^{\mathrm{c}}, x^{\mathrm{u}}\right) \notin \mathcal{W}_{2}$, for all $t \in\left[-t_{0}, t_{0}\right]$.

We observe that this arc has the following properties:

- Suppose that the heteroclinic point $X_{0}=f_{0}^{N_{2}}\left(W_{\text {loc }}^{\mathrm{u}}\left(P_{0}\right)\right) \cap W_{\mathrm{loc}}^{\mathrm{s}}\left(Q_{0}\right)$ above is of the form $X_{0}=\left(x_{0}^{\mathrm{s}}, 0,0^{\mathrm{u}}\right)$. Then, for each $t>0$, the points $X_{t}=\left(x_{0}^{\mathrm{s}}, t, 0^{\mathrm{u}}\right)$ and $Y_{t}=\left(x_{0}^{\mathrm{s}}, 0,0^{\mathrm{u}}\right)$ are homoclinic points associated to $P_{t}$ and $Q_{t}$ (the continuations of $P$ and $Q$ for $f_{t}$ ), respectively. We say that these homoclinic points are the continuations of the heteroclinic point $X_{0}$.

- The foliations $\mathcal{F}^{\mathrm{s}}, \mathcal{F}^{\mathrm{u}}$ and $\mathcal{F}^{\mathrm{c}}$ are $f_{t}$-invariant for all $t$ (recall condition (F1)).

- For each $t$ we can define (in the natural way) projections $\pi_{t}^{\mathrm{u}}, \pi_{t}^{\mathrm{s}}, \pi_{t, P}^{\mathrm{c}}$ and $\pi_{t, Q}^{\mathrm{c}}$. Then $f_{t}^{N_{2}} \circ \pi_{t}^{\mathrm{u}}$ and $f_{t}^{-N_{2}} \circ \pi_{t}^{\mathrm{s}}$ are isometries, and the restrictions to $\gamma$ of $\pi_{t, P}^{\mathrm{c}}$ and $\pi_{t, Q}^{\mathrm{c}}$ are isometries that preserve the natural ordering of $\gamma$ (recall condition (F2)).

- The arc is constructed in such a way that $f_{t}$ is Morse-Smale for every $t<0$ (i.e., the arc has a first bifurcation at $t=0$ ).

- The open set $\mathcal{V}$ defined in (1.1) is contained in $M_{2} \backslash M_{1}$. We say that $\mathcal{V}$ is a neighbourhood of the cycle and define the maximal $f_{t}$-invariant set associated to $\mathcal{V}$ by

$$
\Lambda_{t}=\Lambda_{t}(\mathcal{V})=\bigcap_{n \in \mathbb{Z}} f_{t}^{n}(\mathcal{V})
$$

Due to the existence of the filtration and the choice of $\mathcal{V}$ it follows that

$$
\Omega\left(f_{t}\right)^{\prime}=\Omega\left(f_{t}\right) \cap \mathcal{V} \subset \Lambda_{t} ;
$$

we call $\Omega\left(f_{t}\right)^{\prime}$ the resulting nonwandering set. Hence, from now on we can restrict our attention to the maximal invariant set of $f_{t}$ in $\mathcal{V}$.

\section{RETURNS AND CENTRAL DYNAMICS}

2.1. Returns. A key idea of our construction is to reduce the study of the dynamics of $f_{t}$ in the neighbourhood $\mathcal{V}$ of the cycle to one-dimensional dynamics. For that, having in mind that every nonwandering point created by the bifurcation has some iterate in $\mathcal{V}_{1}$ (excluding the possible nonwandering points in the connexion $\gamma$ ), we analyze the returns of points in $\Lambda_{t} \cap \mathcal{V}_{1}$ to $\mathcal{V}_{1}$ and study the dynamics of such returns in the central direction: relative positions of the returns and expansion or contraction of the derivative of the return map in the central direction. Recall that, in our model, the central direction is $f_{t}$-invariant. Let us now explain all that in detail. 
Consider a point $Z \in \Lambda_{t} \cap \mathcal{V}_{1}$ such that $Z \notin W^{\mathrm{s}}\left(P_{t}\right) \cup W^{\mathrm{s}}\left(Q_{t}\right)$ (since the points $P_{t}$ and $Q_{t}$ do not change with the parameter, from now on we will omit their dependence on $t$ ). Then, after $r_{1}$ positive iterations, $r_{1}=$ $j_{2}+N_{2}+j_{1}+N_{1}$, this point has a first return to $\mathcal{V}_{1}$ as follows:

- $f_{t}^{N_{1}}(Z) \in \mathcal{U}_{P}$

- $f_{t}^{j_{1}+N_{1}}(Z) \in f_{t}^{-N_{2}}\left(\mathcal{V}_{2}\right)$ and $f_{t}^{i+N_{1}}(Z) \in \mathcal{U}_{P}$ for all $i \in\left\{0, \ldots, j_{1}\right\}$,

- $f_{t}^{N_{2}+j_{1}+N_{1}}(Z) \in \mathcal{U}_{Q}$,

- $f_{t}^{j_{2}+N_{2}+j_{1}+N_{1}}(Z) \in \mathcal{V}_{1}$ and $f_{t}^{i+N_{2}+j_{1}+N_{1}}(Z) \in \mathcal{U}_{Q}$ for all $i \in\left\{0, \ldots, j_{2}\right\}$,

where $j_{2}$ is defined to be the minimal one with this property.

It is clear that $j_{1}$ and $j_{2}$ (and thus $r_{1}$ ) depend on $Z$. We will see later that $j_{1}$ and $j_{2}$ tend to infinity as $t$ goes to zero.

Let $Z_{0}=Z$ and $Z_{i}=f_{t}^{i}(Z)$. If possible, we inductively define the successive return times and returning points of $Z$ to $\mathcal{V}_{1}$ by $r_{i}(Z)=r_{1}\left(Z_{r_{i-1}}\right)$ and $Z_{r_{i}}=f_{t}^{r_{i}-r_{i-1}}\left(Z_{r_{i-1}}\right)$.

Condition (F1) and the definition of the arc $\left(f_{t}\right)_{t \in\left[-t_{0}, t_{0}\right]}$ assure that the leaves $\mathcal{F}^{\mathrm{u}}, \mathcal{F}^{\mathrm{s}}$ and $\mathcal{F}^{\mathrm{c}}$ are invariant under $f_{t}^{r_{1}}$; that is,

- $f_{t}^{r_{1}}\left(F^{\mathrm{s}}(Z)\right) \subset F^{\mathrm{s}}\left(Z_{r_{1}}\right)$,

- $F^{\mathrm{u}}\left(Z_{r_{1}}\right) \subset f_{t}^{r_{1}}\left(F^{\mathrm{u}}(Z)\right)$,

- $F^{\mathrm{c}}\left(Z_{r_{1}}\right) \cap f_{t}^{r_{1}}\left(F^{\mathrm{c}}(Z)\right)$ is a curve containing $Z_{r_{1}}$ in its interior.

In particular, if $E^{i}(W)$ is the tangent space to $F^{i}(W)$ at $W$, then we have $D f_{t}^{r_{1}}\left(E^{i}(Z)\right)=E^{i}\left(Z_{r_{1}}\right), i=\mathrm{s}, \mathrm{u}, \mathrm{c}$. We call $E^{\mathrm{s}}, E^{\mathrm{u}}$ and $E^{\mathrm{c}}$ the stable, unstable and central directions, respectively.

Condition (F2) means that, for every $W \in \mathcal{V}_{2}$, the restriction of $D f_{t}^{N_{2}}$ to the central direction is an isometry.

Let $\lambda_{\mathrm{s}}$ (resp. $\beta_{\mathrm{u}}$ ) be the maximum (resp. minimum) of the moduli of the contracting (resp. expanding) eigenvalues of both $P$ and $Q$. Then it is clear that, for small $t$ :

(R1) The restriction of $D f_{t}^{r_{1}}$ to $E^{\mathrm{u}}(Z)$ is an expansion of factor (at least) $\beta_{\mathrm{u}}^{j_{1}+j_{2}}$.

(R2) The restriction of $D f_{t}^{r_{1}}$ to $E^{\mathrm{s}}(Z)$ is a contraction of factor (at least) $\lambda_{\mathrm{S}}^{j_{1}+j_{2}}$.

(R3) The restriction of $D f_{t}^{r_{1}}$ to the one-dimensional bundle $E^{\mathrm{c}}(Z)$ is a multiplication by a factor of order of $\left.\beta^{j_{2}} \lambda^{j_{1}}\left(D f_{t}^{N_{1}}\right)\right|_{E^{c}}(W)$ for some point $W \in \mathcal{V}_{1}$.

Similar considerations can be made for the backward orbit of any point $Z \in \Lambda_{t} \cap \mathcal{V}_{1}$ such that $Z \notin W^{\mathrm{u}}(P) \cup W^{\mathrm{u}}(Q)$.

In view of (R1)-(R3), the hyperbolicity of invariant subsets of $\Lambda_{t}$ depends exclusively on the action of $D f_{t}^{N_{1}}$ on $E^{\mathrm{c}}(W)$ for $W \in \mathcal{V}_{1}$. Let us now analyze the central dynamics of a first return to $\mathcal{V}_{1}$. 
2.2. Central dynamics of returns. Consider now a point $Z=$ $\left(z^{\mathrm{s}}, z^{\mathrm{c}}, z^{\mathrm{u}}\right) \in \mathcal{V}_{1}$ such that $z^{\mathrm{c}} \in I$ and a first return $r_{1}=j_{2}+N_{2}+j_{1}+N_{1}$ of $Z$ to $\mathcal{V}_{1}$ as above. Using the product structure of the cycle and the definition of the unfolding we see that if $Z_{r_{1}}=\left(z_{1}^{\mathrm{s}}, z_{1}^{\mathrm{c}}, z_{1}^{\mathrm{u}}\right)=f_{t}^{j_{2}+N_{2}+j_{1}+N_{1}}(Z)$, then

$$
\begin{aligned}
z_{1}^{\mathrm{c}} & =\left(f_{t}^{j_{2}+N_{2}+j_{1}+N_{1}}(Z)\right)^{\mathrm{c}}=\beta^{j_{2}}\left(\lambda^{j_{1}} f_{0}^{N_{1}}\left(0^{\mathrm{s}}, z^{\mathrm{c}}, 0^{\mathrm{u}}\right)+t\right) \\
& =\beta^{j_{2}} \lambda^{j_{1}} f_{0}^{N_{1}}\left(0^{\mathrm{s}}, z^{\mathrm{c}}, 0^{\mathrm{u}}\right)+\beta^{j_{2}} t,
\end{aligned}
$$

where

$$
\beta^{j_{2}}\left(\lambda^{j_{1}} f_{0}^{N_{1}}\left(0^{\mathrm{s}}, z^{\mathrm{c}}, 0^{\mathrm{u}}\right)+t\right) \in I
$$

Thus we have

$$
\lambda^{j_{1}} f_{0}^{N_{1}}\left(0^{\mathrm{s}}, z^{\mathrm{c}}, 0^{\mathrm{u}}\right)+t>0 \Rightarrow \lambda^{j_{1}} f_{0}^{N_{1}}\left(0^{\mathrm{s}}, z^{\mathrm{c}}, 0^{\mathrm{u}}\right) \in(-t, 0] .
$$

Since $f_{0}^{N_{1}}\left(0^{\mathrm{s}}, z^{\mathrm{c}}, 0^{\mathrm{u}}\right) \in J=f_{0}^{N_{1}}(I)$ we see immediately that there is $m_{t}$ independent of the point $\left(0^{\mathrm{s}}, z^{\mathrm{c}}, 0^{\mathrm{u}}\right)$ in $I$ such that

$$
j_{1}=m_{t}+m, \quad m \geq 0, \quad \lambda^{m_{t}} \simeq t .
$$

Similarly, there is $n_{t}$ independent of the point $\left(0^{\mathrm{s}}, z^{\mathrm{c}}, 0^{\mathrm{u}}\right)$ of $I$ such that

$$
j_{2}=n_{t}+n, \quad n \geq 0, \quad \beta^{n_{t}} \simeq 1 / t .
$$

Observe that points close to $Z$ have the same type of return as $Z$. This return defines the following map from a subinterval $K_{n, m}^{t}$ (that we consider maximal) of $I$ into $I$ :

$$
\begin{aligned}
G_{n, m}^{t}: K_{n, m}^{t} \rightarrow I, \quad G_{n, m}^{t}(z) & =\beta^{n_{t}+n}\left(\lambda^{m_{t}+m} \Pi\left(z^{\mathrm{c}}\right)+t\right) \\
& =\left(\beta^{n_{t}} \lambda^{m_{t}}\right) \beta^{n} \lambda^{m} \Pi\left(z^{\mathrm{c}}\right)+\beta^{n_{t}} \beta^{n} t .
\end{aligned}
$$

where $\Pi: I \rightarrow J$ is the restriction of $f_{0}^{N_{1}}$ to $I$.

We will select parameters $t>0$ (see Section 5 ) such that

$$
\beta^{n_{t}} \lambda^{m_{t}} \simeq 1 \text { and } \beta^{n_{t}} t \simeq 1
$$
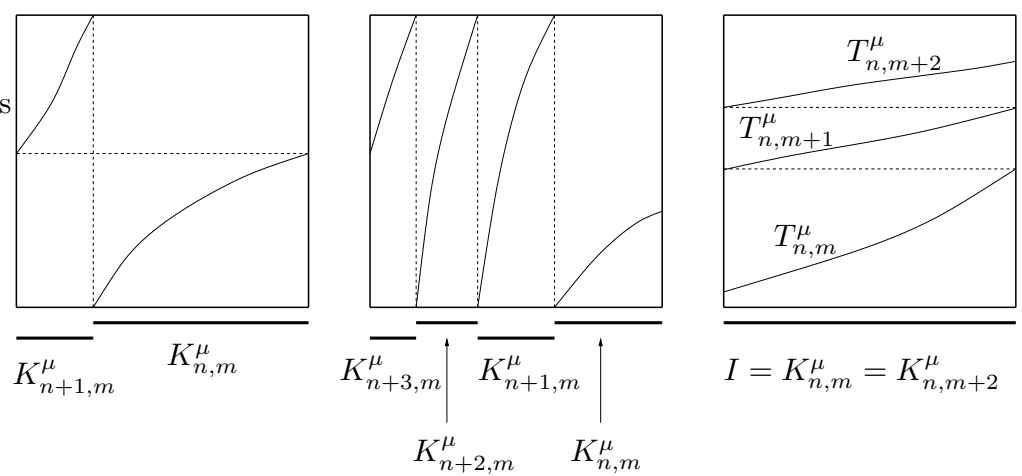

Fig. 4. The maps $T_{n, m}^{\mu}$ and the sets $K_{n, m}^{\mu}$ : different possibilities 
For these parameters the maps $G_{n, m}^{t}$ are close to maps of the form

$$
T_{n, m}^{\mu}(z)=\beta^{n}\left(\lambda^{m} \Pi(z)+1+\mu\right)
$$

for some small $|\mu|$. In the next section we study the dynamics of the maps $T_{n, m}^{\mu}$.

\section{THE ONE-DIMENSIONAL MODEL FOR THE RETURNS}

Consider $0<\lambda<1<\beta$ and the linear maps defined on $\mathbb{R}$ by $x \mapsto \lambda x$ and $x \mapsto \beta x$. Given $a, b \in(1,2)$ consider their fundamental domains $J=$ $[-a,-\lambda a]$ and $I=[\alpha b, b], \alpha=\beta^{-1}$.

Consider also a strictly increasing differentiable map $\Pi$ from $I$ to $J$ with $\Pi(I)=J$ and, for $\mu$ in a small neighbourhood of 0 , the family of maps

$$
H^{\mu}:[-2,0] \rightarrow[-2,2], \quad H^{\mu}(y)=y+1+\mu .
$$

We now define the parametrized family of endomorphisms $\mathcal{T}^{\mu}=\left(T_{n, m}^{\mu}\right)_{n, m \geq 0}$ by

$$
T_{n, m}^{\mu}=\beta^{n} \circ H^{\mu} \circ \lambda^{m} \circ \Pi, \quad n, m \geq 0,
$$

defined for each pair $n, m, n, m \geq 0$, from a subinterval of $I$ into $I$. In general, these maps are not defined in the whole $I$, so for each pair $(n, m)$ we consider the maximal subinterval $K_{n, m}^{\mu}$ of $I$ where $T_{n, m}^{\mu}$ is defined. We observe that there are choices of $(n, m)$ such that $K_{n, m}^{\mu}=\emptyset$ (see Lemmas 3.3 and 3.4).

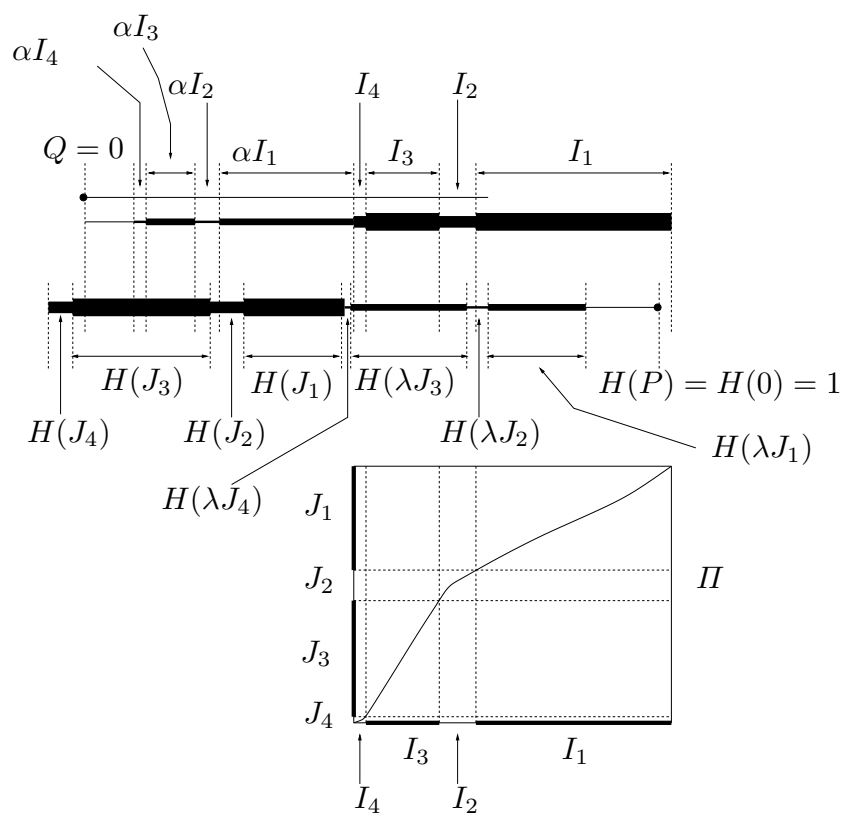

Fig. 5. The one-dimensional model 
We say that $\Pi$ and $H^{\mu}$ are the transition and the unfolding maps of the iterated function system $\mathcal{T}^{\mu}$.

In our construction we assume that the map $\Pi$ from $I$ to $J$ has the properties listed below. There is a partition of $I$ into four closed subintervals, denoted by $I_{1}, I_{2}, I_{3}$ and $I_{4}$, with pairwise disjoint interiors, such that $\alpha b \in I_{4}, b \in I_{1}$, and $x_{4} \leq x_{3} \leq x_{2} \leq x_{1}$ for any points $x_{4}, x_{3}, x_{2}$ and $x_{1}$ with $x_{i} \in I_{i}$. We let $J_{i}=\Pi\left(I_{i}\right)$. Thus $J_{i}$ is a partition of $J$. We require that $\Pi$ and the subdivisions of $I$ and $J$ above satisfy the following conditions, illustrated in Figure 5.

Inclusion properties:

(E1) The interior of $H^{0}\left(J_{3}\right)$ contains $\beta^{-1} I_{3}$.

(E2) $\quad H^{0}\left(J_{1}\right)$ is contained in the interior of $\beta^{-1} I_{1}$.

(E3) The right endpoint of $H^{0}\left(J_{3}\right)$ is less than the left endpoint of $\beta^{-1} I_{1}$ (in particular, $\beta^{-1} I_{2}$ is not contained in $H^{0}\left(J_{3}\right)$ ).

(E4) The interior of $H^{0}\left(\lambda J_{3}\right)$ contains $I_{3} \cup I_{4}$.

(E5) The right endpoint of $H^{0}\left(\lambda J_{3}\right)$ is less than the right endpoint of $I_{2}$ (in particular, $I_{2}$ is not contained in $H^{0}\left(\lambda J_{3}\right)$ ).

(E6) The right endpoint of $I_{2}$ is less than the left endpoint of $H^{0}\left(\lambda J_{1}\right)$ (in particular, $H^{0}\left(\lambda J_{1}\right)$ is contained in the interior of $\left.I_{1}\right)$.

(E7) Every point of the interval $H^{0}\left(J_{4}\right)$ is less than zero (in particular, $\left.0 \in H^{0}\left(J_{3}\right)\right)$.

Properties of derivatives. Distortion properties:

(E8) The map $\Pi$ is a contraction of factor (at least) $\varrho^{\mathrm{s}}<\alpha=\beta^{-1}<1$ in $I_{1}$ and an expansion of factor (at least) $\varrho^{\mathrm{u}}>\lambda^{-1}>1$ in $I_{3}$.

(E9) $T_{0,1}^{0}$ has exactly two fixed points in the interior of $I_{2}$, say $\widetilde{A}$ and $\widetilde{R}$, $\widetilde{A}<\widetilde{R}$, which are hyperbolic and whose derivatives $D T_{0,1}^{0}(\widetilde{A})=\lambda_{A}$ and $D T_{0,1}^{0}(\widetilde{R})=\beta_{R}$ satisfy

$$
0<\lambda_{A}<1<\beta_{R}
$$

Moreover, there is $\xi>1$ such that

$$
\min \left\{\frac{\lambda_{A}^{2}}{2\left(1-\beta_{R}^{-1}\right)\left(1+\lambda_{A}\right) \beta_{R}^{2}}, \frac{\lambda_{A}^{2}}{2\left(1-\lambda_{A}\right)\left(1+\beta_{R}^{-1}\right) \beta_{R}^{2}}\right\}>2 \xi .
$$

There are $N_{3}>1$ and points $\widetilde{C}$ and $\widetilde{D}$ with $\widetilde{A}<\widetilde{C}<\widetilde{D}<\widetilde{R}$ and $\widetilde{C}-\widetilde{A}=\widetilde{R}-\widetilde{D}$ such that

- $T_{0,1}^{0}$ is linear in $[\widetilde{A}, \widetilde{C}]$ and $[\widetilde{D}, \widetilde{R}]$.

- $\left(T_{0,1}^{0}\right)^{N_{3}}\left(\left[\beta_{R}^{-1} \widetilde{D}, \widetilde{D}\right]\right)=\left[\lambda_{A} \widetilde{C}, \widetilde{C}\right]$,

- $\frac{1-\lambda_{A}}{1-\beta_{R}} \leq D\left(\left(T_{0,1}^{0}\right)^{N_{3}}\right)(x) \leq 2 \frac{1-\lambda_{A}}{1-\beta_{R}}$. 
Fixed point properties:

(E10) $T_{1,0}^{0}$ has no fixed points in $I_{2}$.

(E11) $\quad T_{1,0}^{0}(y)>T_{0,1}^{0}(y)$ for all $y \in I_{2}$.

(E12) $\quad \widetilde{R}=\beta H^{0}(\Pi(\widetilde{A}))=T_{1,0}^{0}(\widetilde{A})$.

Finally, we also assume that

(E13) $\quad \beta>131$.

Let us make some comments about these conditions.

REMARK 3.1. Conditions (E1)-(E11) and (E13) are open properties. Thus there is $\mu_{0}>0$ such that these conditions hold for $H^{\mu}, T_{1,0}^{\mu}$ and $T_{0,1}^{\mu}$ for all $\mu \in\left(-\mu_{0}, \mu_{0}\right)$. Observe that to get the second part of (E9) it is enough to take the eigenvalues $\beta_{R}$ and $\lambda_{A}$ close enough to 1 .

REMARK 3.2. • The maps $T_{n, m}^{\mu}$ are increasing for all $(n, m)$ for every $|\mu|$ small enough. Moreover, $T_{0,1}^{\mu}$ (for small $\left.|\mu|\right)$ has no fixed points in $\left(\widetilde{A}_{\mu}, \widetilde{R}_{\mu}\right)$.

- We can assume that for every $\mu \in\left(-\mu_{0}, \mu_{0}\right)$ there are defined the continuations of the hyperbolic points $\widetilde{A}$ and $\widetilde{R}$ of $T_{0,1}^{0}$ for all $T_{0,1}^{\mu}$, denoted by $\widetilde{A}_{\mu}$ and $\widetilde{R}_{\mu}$. Then $T_{1,0}^{\mu}\left(\widetilde{A}_{\mu}\right) \geq \widetilde{R}_{\mu}$ for every $\mu \geq 0$.

Proof. The first item of the remark is obvious. For the second one we refer the reader to Lemma 3.12, where we will prove a stronger version of this remark: the map $U(\mu)=T_{1,0}^{\mu}\left(\widetilde{A}_{\mu}\right)-\widetilde{R}_{\mu}$ has positive derivative at $\mu=0$.

Let us now explain how these conditions can be achieved by choosing appropriate lengths for the subintervals $I_{i}$ and $J_{i}$ and by making some assumptions on $\lambda, \alpha=\beta^{-1}, a$ and $b$. Write

$$
\begin{array}{ll}
I_{1}=\left[\left(1-\varepsilon_{1}\right) a, a\right], & J_{1}=\left[\left(-1+\delta_{1}\right) b,-\lambda b\right], \\
I_{2}=\left[\left(1-\varepsilon_{2}\right) a,\left(1-\varepsilon_{1}\right) a\right], & J_{2}=\left[\left(-1+\delta_{2}\right) b,\left(-1+\delta_{1}\right) b\right], \\
I_{3}=\left[\left(1-\varepsilon_{3}\right) a,\left(1-\varepsilon_{2}\right) a\right], & J_{3}=\left[\left(-1+\delta_{3}\right) b,\left(-1+\delta_{2}\right) b\right], \\
I_{4}=\left[\alpha a,\left(1-\varepsilon_{3}\right) a\right], & J_{4}=\left[-b,\left(-1+\delta_{3}\right) b\right],
\end{array}
$$

where

$$
0<\varepsilon_{1}<\varepsilon_{2}<\varepsilon_{3}<1-\alpha \text { and } 0<\delta_{3}<\delta_{2}<\delta_{1}<1-\lambda .
$$

It is not difficult to see that if $a$ and $b$ are close enough to 1 then conditions (E1)-(E6) are satisfied if one chooses:

(D2) $\quad(1-\alpha) \lambda<\varepsilon_{2}<\frac{1-\alpha}{\lambda^{-1}-\alpha}$ (in fact, $\varepsilon_{2}$ must be chosen near this maximal value),

$$
\lambda^{2}<\varepsilon_{1}<1+\alpha^{-1}\left(\lambda^{-1} \varepsilon_{2}-1\right),
$$


(D4) $\delta_{3}<\frac{\alpha+\lambda-1}{\lambda}$,

(D5) $\quad 1-\lambda^{-1} \varepsilon_{2}<\delta_{2}<\alpha\left(1-\varepsilon_{1}\right)$,

(D6) $1-\lambda^{-1} \varepsilon_{1}<\delta_{1}<1-\lambda$.

In fact, after a straightforward calculation it is easy to check that (D1)-(D6) imply (E1)-(E6) when $a=b=1$. Since (E1)-(E6) are open conditions the same holds when $a$ and $b$ are close to 1 .

Observe that (E7) is automatically satisfied if $b$ is close to 1 and $\delta_{3}$ is sufficiently small $\left(\delta_{3}<1-1 / b\right)$.

It is easy to verify that if we choose $\varepsilon_{i}$ and $\delta_{i}$ as above, $i=1, \ldots, 4$, and $b / a$ close to 1 then the following conditions are also satisfied:

$$
\frac{\left|J_{1}\right|}{\left|I_{1}\right|} \alpha^{-1}<\bar{\varrho}<1<\varrho<\frac{\left|J_{3}\right|}{\left|I_{3}\right|} \lambda
$$

for some $\bar{\varrho}$ and $\varrho$, where $|K|$ denotes the length of the interval $K$. These conditions imply that we can take a transition $\Pi$ from $I$ to $J$ satisfying (E8) and (E9).

Let us now explain how one can obtain (E12). Observe that this condition is equivalent to

$$
\alpha \widetilde{R}=\Pi(\widetilde{A})+1=\lambda^{-1}(\widetilde{A}-1)+1,
$$

where the last equality follows from the fact that $\widetilde{A}$ is a fixed point of $T_{0,1}^{0}=H^{0} \circ f \circ \Pi$. Therefore, if we write $\widetilde{R}=\widetilde{A}+\tau$ for some $\tau>0$, the previous formula gives

$$
\begin{aligned}
\tau & =\widetilde{A}\left(-1+\alpha^{-1} \lambda^{-1}\right)-\alpha^{-1} \lambda^{-1}+\alpha^{-1}, \quad \text { i.e. }, \\
\widetilde{R} & =\widetilde{A} \alpha^{-1} \lambda^{-1}-\alpha^{-1} \lambda^{-1}+\alpha^{-1},
\end{aligned}
$$

which determines $\tau$ depending on $\widetilde{A}$.

On the other hand, by construction, the points $\widetilde{A}$ and $\widetilde{R}$ belong to $I_{2} \backslash H^{0}\left(\lambda J_{3}\right)$, thus $\widetilde{A}$ is to the right of the left extreme of $I_{2}, a\left(1-\varepsilon_{2}\right)$. Thus $\widetilde{A}=a\left(1-\varepsilon_{2}+\xi\right)$ for some $\xi>0$. Now it is enough that $\widetilde{A}$ and $\widetilde{R}$ satisfy the following inequalities obtained by replacing the value of $\widetilde{R}$ in (3.2) (the first one meaning that $\widetilde{A}$ is to the right of $H^{0}\left(\lambda J_{3}\right)$ and the second one meaning that $\widetilde{R}$ is to the left of $I_{1}$ ),

$$
\begin{aligned}
& \lambda b\left(-1+\delta_{2}\right)+1<a\left(1-\varepsilon_{2}+\xi\right), \\
& a\left(1-\varepsilon_{2}+\xi\right) \alpha^{-1} \lambda^{-1}-\alpha^{-1} \lambda^{-1}+\alpha^{-1}<a\left(1-\varepsilon_{1}\right) .
\end{aligned}
$$

If we take

$$
\xi=a^{-1} \lambda b\left(-1+\delta_{2}\right)+a^{-1}+\varepsilon_{2}-1,
$$

which turns the first inequality of (3.3) into equality, then the second in- 
equality in (3.3) is equivalent to

$$
\alpha^{-1} \delta_{2}<a b^{-1}\left(1-\varepsilon_{1}\right)+\alpha^{-1}\left(1-b^{-1}\right),
$$

which is guaranteed by condition (D5) if $a$ and $b$ are close to 1 . Thus, if we take $\xi$ greater than $a^{-1} \lambda b\left(-1+\delta_{2}\right)+a^{-1}+\varepsilon_{2}-1$ but near this value, then (3.3) is satisfied.

Observe that (E13) is compatible with (D1). Also, (E5) and (E6) imply that $H^{0}\left(\lambda \Pi\left(I_{2}\right)\right) \cap I_{2} \neq \emptyset$ and (E1)-(E3) imply that $\beta H^{0}\left(\Pi\left(I_{2}\right)\right) \cap I_{2} \neq \emptyset$.

Condition (E11) means that

$$
\beta H^{0}(\Pi(y))>H^{0}(\lambda \Pi(y)) \text { for every } y \in I_{2},
$$

in other words,

$$
(\beta-\lambda) \Pi(y)+(\beta-1)>0 \quad \text { for all } y \in I_{2} .
$$

Now $\Pi(y) \in J_{2}$ for all $y \in I_{2}$, therefore (by (D5))

$$
\Pi(y) \geq b\left(-1+\delta_{2}\right)>b\left(-1+1-\lambda^{-1} \varepsilon_{2}\right)=-b \lambda^{-1} \varepsilon_{2} .
$$

Thus to get (3.4) it is enough that

$$
(\beta-\lambda) \Pi(y)+(\beta-1) \geq(\beta-\lambda)\left(-b \lambda^{-1} \varepsilon_{2}\right)+(\beta-1)>0 .
$$

Thus (3.4) is satisfied if

$$
\varepsilon_{2}<\frac{\beta-1}{\beta-\lambda} \lambda b^{-1}=\frac{1-\alpha}{\lambda^{-1}-\alpha} b^{-1},
$$

a condition that is satisfied for $b$ close to 1 (recall (D2)).

Finally, observe that (E10) is compatible with the previous conditions.

3.1. Transitions and iterated function systems. In this section we study the iterations and compositions of the maps $T_{n, m}^{\mu}$,

$$
T_{n, m}^{\mu}: K_{n, m}^{\mu} \rightarrow I, \quad x \mapsto \beta^{n} H^{\mu}\left(\lambda^{m} \Pi(x)\right)=\beta^{n} \lambda^{m} \Pi(x)+\beta^{n}(1+\mu) .
$$

We begin by stating some lemmas about the localization of the iterates of points $x \in I$ under the maps $T_{n, m}^{\mu}$.

Given $x \in I$ we define $a(x) \in\{1,2,3,4\}$ as follows. If $x \in I_{j}, j=1$ or 3 , then we let $a(x)=j$. If $x \in \operatorname{int}\left(I_{j}\right), j=2$ or 4 , we let $a(x)=j$. Finally, $a(\alpha)=4$.

Lemma 3.3. Let $\mu \in\left(-\mu_{0}, \mu_{0}\right)$ and consider a map $T_{n, m}^{\mu}$ with $m \geq 2$ such that $K_{n, m}^{\mu} \neq \emptyset$. Then $n=0$. Moreover, $K_{0, m}^{\mu}=I$ and $a\left(T_{0, m}^{\mu}(x)\right)=1$ for all $x \in I$.

Proof. Since $m \geq 2, \lambda^{m} \Pi(x)$ is to the right of $\lambda J_{1}$ for every $x$ in $I$. Thus $H^{\mu}\left(\lambda^{m} \Pi(x)\right)$ is also to the right of $H^{\mu}\left(\lambda J_{1}\right)$. By (E6) and Remark 3.1, $H^{\mu}\left(\lambda J_{1}\right) \subset I_{1}$, which implies all the assertions in the lemma. 
Lemma 3.4. Consider $\mu \in\left(-\mu_{0}, \mu_{0}\right)$ and $x \in I_{1} \cup I_{2}$ such that $T_{n, m}^{\mu}(x)$ is defined (i.e., $\left.x \in K_{n, m}^{\mu}\right)$. Then:

(1) If $m=1$ then $n=0$ and $a\left(T_{0,1}^{\mu}(x)\right)=1$ if $x \in I_{1}$, while it is 1 or 2 if $x \in I_{2}$.

(2) If $m=0$ then $n=1$ and $a\left(T_{1,0}^{\mu}(x)\right)=1$ if $x \in I_{1}$, while it is 1 or 2 if $x \in I_{2}$.

(3) If $m \geq 2$ then $n=0$ and $a\left(T_{0, m}^{\mu}(x)\right)=1$.

Proof. Suppose that $x \in I_{1} \cup I_{2}$ and $m=1$. Observe that $H^{\mu}(\lambda \Pi(x))$ is to the right of $H^{\mu}\left(\lambda J_{3}\right)$. Thus, by (E3), $H^{\mu}(\lambda \Pi(x)) \in I_{2} \cup I_{1}$. Thus $n=0$. This also implies that $a\left(T_{0,1}^{\mu}(x)\right)=1$ or 2 . To end the proof of (1) it remains to see that this number is 1 if $x \in I_{1}$. But this follows immediately from (E6).

To prove (2) observe that if $m=0$ then, by (E1) and since $x \in I_{1} \cup I_{2}$, it follows that $H^{\mu}(\Pi(x))$ is to the right of $\beta^{-1} I_{3}$ and, by (E2), it is to the left of $I_{4}$, thus we must iterate once to return to $I$, that is, $n=1$. Also (E2) implies that $a\left(T_{1,0}^{\mu}(x)\right)=1$ if $x \in I_{1}$, and (E1) and (E2) imply that $a\left(T_{1,0}^{\mu}(x)\right)=1$ or 2 if $x \in I_{2}$.

Finally, item (3) follows from Lemma 3.3.

The next lemma follows from conditions (E2), (E4), (E6) and (E7) and its proof is analogous to the one of the previous lemma.

Lemma 3.5. Consider $\mu \in\left(-\mu_{0}, \mu_{0}\right)$ and $x \in I_{4}$ such that $T_{n, m}^{\mu}(x)$ is defined. Then $m \geq 1$. Moreover, if $m=1$ then $n=1$, and if $m>1$ then $n=0$. In any case, $a\left(T_{n, m}^{\mu}(x)\right)=1$.

Proof. By (E7) we have $m \geq 1$. If $m=1$ then, by (E2) and (E4), we have $H^{\mu}\left(\lambda J_{4}\right) \subset \beta^{-1} I_{1}$, thus $n=1$ and $a\left(T_{1,1}^{\mu}\right)=1$. If $m>1$ then, by (E6), $H^{\mu}\left(\lambda^{m} J_{4}\right) \subset I_{1}$, thus $n=0$ and $a\left(T_{0,1}^{\mu}\right)=1$.

By Lemmas 3.4 and 3.5, if we are interested in returns of points of $I_{1} \cup I_{2} \cup I_{4}$ under $\left(T_{n, m}^{\mu}\right)$ we only need to consider three kinds of maps, $T_{1,0}^{\mu}$, $T_{1,1}^{\mu}$ and $T_{0, m}^{\mu}$, where $m \geq 1$.

3.2. Orbits and itineraries. For a fixed $\mu \in\left(-\mu_{0}, \mu_{0}\right)$ consider the pseudo-group generated by the family $\mathcal{T}^{\mu}=\left(T_{n, m}^{\mu}\right)$ of maps. Given a point $z \in I$ we say that a sequence $\left(n_{j}, m_{j}\right)_{j=1}^{k}, n_{j}, m_{j} \geq 0$, of pairs of integers is admissible for $z$ if the sequence of points

$$
z_{n_{j}, m_{j}}^{\mu}=T_{n_{j}, m_{j}}^{\mu}\left(z_{n_{j-1}, m_{j-1}}^{\mu}\right)
$$

is well defined for every $j=1, \ldots, k$, where $z_{n_{0}, m_{0}}=z$. In this case we say that $\left(z_{n_{j}, m_{j}}^{\mu}\right)_{j}$ is the $\mathcal{T}^{\mu}$-orbit of $z$ for the sequence $\left(n_{j}, m_{j}\right)_{j}$. To the orbit $\left(z_{n_{j}, m_{j}}^{\mu}\right)_{j}$ we associate its itinerary defined by

$$
i_{n_{j}, m_{j}}^{\mu}(z)=a\left(z_{n_{j}, m_{j}}^{\mu}\right) .
$$


Lemma 3.6. Given $z \in I$ and an orbit $\left(z_{n_{j}, m_{j}}^{\mu}\right)_{j}$ of $z$, one of the following situations hold:

- There is $j_{0}$ such that $i_{n_{j}, m_{j}}^{\mu}(z)=1$ for all $j \geq j_{0}$.

- There is $j_{0}$ such that $i_{n_{j}, m_{j}}^{\mu}(z)=2$ for all $j \geq j_{0}$.

- $i_{n_{j}, m_{j}}^{\mu}(z)=3$ for all $j \geq 0$.

Proof. On the one hand, by Lemma 3.4,

- if $i_{n_{j}, m_{j}}^{\mu}(z)=1$ then $i_{n_{j+k}, m_{j+k}}^{\mu}(z)=1$ for all $k \geq 0$,

- if $i_{n_{j}, m_{j}}^{\mu}(z)=2$ then $i_{n_{j+k}, m_{j+k}}^{\mu}(z)=1$ or 2 for all $k \geq 0$.

On the other hand, by Lemma 3.5,

- if $i_{n_{j}, m_{j}}^{\mu}(z)=4$ then $i_{n_{j+1}, m_{j+1}}^{\mu}(z)=1$.

These assertions imply the lemma.

3.3. Localization of the nonwandering set of $\mathcal{T}^{\mu}$. We say that a point $z$ belongs to the nonwandering set of $\mathcal{T}^{\mu}$, denoted by $\Omega\left(\mathcal{T}^{\mu}\right)$, if for every neighbourhood $V$ of $z$ there is a point $w \in V$ and an admissible sequence $\left(n_{j}, m_{j}\right)_{j=1}^{k}, k \geq 1$, for $w$ such that $w_{n_{k}, m_{k}}^{\mu} \in V$. For each $i \in$ $\{1, \ldots, 4\}$ let $\Omega_{i}^{\mu}=\Omega\left(\mathcal{T}^{\mu}\right) \cap I_{i}$.

The $\omega$-limit set of $x$ for $\mathcal{T}^{\mu}$, denoted by $\omega^{\mu}(x)$, is the set of points $z$ such that for every neighbourhood $V$ of $z$ there is a $\mathcal{T}^{\mu}$-orbit $x_{n_{j}, m_{j}}^{\mu}$ of $x$ such that $x_{n_{k}, m_{k}}^{\mu} \in V$ for some $k>0$.

Proposition 3.7. Let $\mu \in\left(-\mu_{0}, \mu_{0}\right)$.

(a) If $z \in \Omega_{1}^{\mu}$ then $z \in \omega^{\mu}(x)$ for every $x \in I_{1}$.

(b) If $z \in \Omega_{2}^{\mu}$ then $z \in\left[\widetilde{A}_{\mu}, \widetilde{R}_{\mu}\right]$. Moreover, if $\mu \geq 0$ then $z=\widetilde{A}_{\mu}$ or $\widetilde{R}_{\mu}$.

(c) If $z \in \Omega_{3}^{\mu}$ then there is a $\mathcal{T}^{\mu}$-orbit $z_{n_{j}, m_{j}}^{\mu}$ of $z$ such that $i_{n_{j}, m_{j}}^{\mu}(z)=3$ for all $j \geq 0$.

(d) $\Omega_{4}^{\mu}=\emptyset$.

Write now $\beta \varrho^{\mathrm{s}}=\bar{\varrho}<1$ and $\lambda \varrho^{\mathrm{u}}=\varrho>1$, where $\varrho^{\mathrm{s}}$ and $\varrho^{\mathrm{u}}$ are the constants in $(\mathrm{E} 8)$.

(e) Let $x \in I_{1}$ and suppose that $x_{n, m}^{\mu} \in I_{1}$. Then $n=0$ or 1 and

$$
0<\left(T_{n, m}^{\mu}\right)^{\prime}(x)<\bar{\varrho}<1 .
$$

(f) Let $x \in I_{3}$ and suppose that $x_{n, m}^{\mu} \in I_{3}$. Then $m=0$ or 1 and

$$
\left(T_{n, m}^{\mu}\right)^{\prime}(x)>\varrho>1 \text {. }
$$

Proof. Items (c) and (d) follow immediately from Lemma 3.6.

To prove (a), first observe that if $z \in \Omega_{1}^{\mu}$ then $z \in \operatorname{int}\left(I_{1}\right)$ (this follows immediately from conditions (E2) and (E6)). We need the following fact whose proof (which is omitted here) also follows easily from (E2) and (E6). 
FACT 3.8. Let $\left(n_{j}, m_{j}\right)_{j}$ be an admissible sequence for $x \in I_{1}$. Then $\left(n_{j}, m_{j}\right)_{j}$ is an admissible sequence for any point of $I_{1}$.

Consider now small $\varepsilon>0$ and a neighbourhood $V_{\varepsilon}=(z-\varepsilon, z+\varepsilon)$ of $z$ in the interior of $I_{1}$. Since $z \in \Omega\left(\mathcal{T}^{\mu}\right)$ there are $w \in V_{\varepsilon}$ and an admissible sequence $\left(n_{j}, m_{j}\right)_{j=1}^{k}$ for $w$ such that

$$
\bar{\varrho}^{k}<\varepsilon / 2 \text { and }\left|w_{n_{k}, m_{k}}^{\mu}-z\right|<\varepsilon / 2 .
$$

Take now any $x \in I_{1}$. By Fact 3.8, the orbit $x_{n_{j}, m_{j}}^{\mu}$ is well defined. By (E8) and since $n_{j}=0$ or 1 and $m_{j} \geq 0$ (recall Lemma 3.4), we have

$$
\left|w_{n_{k}, m_{k}}^{\mu}-x_{n_{k}, m_{k}}^{\mu}\right| \leq\left(\beta \varrho^{\mathrm{s}}\right)^{k}=\bar{\varrho}^{k}<\varepsilon / 2 .
$$

Thus $\left|z-x_{n_{k}, m_{k}}^{\mu}\right|<\varepsilon$. Since this construction can be done for every $\varepsilon>0$ and every point $x \in I_{1}$, one has $z \in \omega^{\mu}(x)$ for every $x \in I_{1}$, ending the proof of (a).

To prove the first part of (b), $\Omega_{2}^{\mu} \subset\left[\widetilde{A}_{\mu}, \widetilde{R}_{\mu}\right]$, recall that, by Lemma 3.4, if $w \in I_{2}$ and $w_{n, m}^{\mu} \in I_{2}$ then $n, m \in\{0,1\}$ and $n+m=1$. Thus there are two kind of returns of points of $I_{2}$ to $I_{2}$ : returns under $T_{1,0}^{\mu}$ and under $T_{0,1}^{\mu}$.

Observe also that every nonwandering point of $I_{2}$ belongs to its interior. This follows from the fact that the right extreme of $I_{2}$ always returns to the interior of $I_{1}$ and the left extreme of $I_{2}$ always returns to the interior of $I_{2}$ or of $I_{1}$ (see Figure 5).

We now need the following remark, which is an immediate consequence of (E9) and (E11).

REMARK 3.9. Let $x \in I_{2}$ be such that $x<\widetilde{A}_{\mu}$ or $x>\widetilde{R}_{\mu}$. Then $T_{1,0}^{\mu}(x)>x$ and $T_{0,1}^{\mu}(x)>x$.

Remark 3.9, (E9), (E11) and (E12) imply the following.

Remark 3.10. Let $x \in I_{2}$ with $x<\widetilde{A}_{\mu}$. Then $T_{0,1}^{\mu}(x)<\widetilde{A}_{\mu}$. Moreover, if $T_{1,0}^{\mu}(x)>\widetilde{A}_{\mu}$ then $x_{n_{j}, m_{j}}^{\mu}>\widetilde{A}_{\mu}$ for every admissible sequence $\left(n_{j}, m_{j}\right)_{j}$ for $x$ with $n_{1}=1$ and $m_{1}=0$.

Suppose that $z<\widetilde{A}_{\mu}$ or $z>\widetilde{R}_{\mu}$. By Remark 3.9 there is a small $\varepsilon>0$ such that

$T_{1,0}^{\mu}(y)>z+\varepsilon \quad$ and $\quad T_{0,1}^{\mu}(y)>z+\varepsilon \quad$ for all $y \in V_{\varepsilon}=(z-\varepsilon, z+\varepsilon) \subset I_{2}$, where $z+\varepsilon<\widetilde{A}_{\mu}$ if $z<\widetilde{A}_{\mu}$ and $z-\varepsilon>\widetilde{R}_{\mu}$ if $z>\widetilde{R}_{\mu}$.

Take now $x \in V_{\varepsilon}$ and an admissible sequence $\left(n_{j}, m_{j}\right)_{j=1}^{k}$ for $x$ such that $x_{n_{k}, m_{k}}^{\mu} \in V_{\varepsilon}$. By Lemma $3.4, i_{n_{j}, m_{j}}^{\mu}(x)=2$ for all $j$, and, by the choice of $V_{\varepsilon}$, $x_{n_{1}, m_{1}}>z+\varepsilon$. Moreover, by Remark 3.10, if $z<\widetilde{A}_{\mu}$ then $x_{n_{1}, m_{1}}^{\mu}<\widetilde{A}_{\mu}$.

Inductively, we have $x_{n_{j}, m_{j}}^{\mu}>z+\varepsilon$. Moreover, if $z<\widetilde{A}_{\mu}$ then $x_{n_{j}, m_{j}}^{\mu}<\widetilde{A}_{\mu}$. In both cases, $x_{n_{j}, m_{j}}^{\mu} \notin V_{\varepsilon}$ for all $j=\{1, \ldots, k\}$, which is a contradiction. 
We now prove the second part of (b), i.e., if $\mu \geq 0$ then $\Omega\left(\mathcal{T}^{\mu}\right) \cap$ $\left(\widetilde{A}_{\mu}, \widetilde{R}_{\mu}\right)=\emptyset$. Consider any $x \in\left(\widetilde{A}_{\mu}, \widetilde{R}_{\mu}\right)$. Then, by (E9) and Remark 3.2, $T_{0,1}^{\mu}(x)<x$ for all $x \in\left(\widetilde{A}_{\mu}, \widetilde{R}_{\mu}\right)$, thus there is a neighbourhood $V_{\varepsilon}=$ $(z-\varepsilon, z+\varepsilon) \subset\left(\widetilde{A}_{\mu}, \widetilde{R}_{\mu}\right)$ such that

$$
T_{0,1}^{\mu}(y)<z-\varepsilon \quad \text { for all } y \in V_{\varepsilon} .
$$

Consider an admissible sequence $\left(n_{j}, m_{j}\right)_{j=1}^{k}$ for $x$ with $x_{n_{k}, m_{k}}^{\mu} \in V_{\varepsilon}$. By Lemma $3.4, i_{n_{j}, m_{j}}^{\mu}(x)=2$ for all $j$. Moreover, observing that if $x>\widetilde{A}_{\mu}$ then $T_{1,0}^{\mu}(x)$ and $T_{0,1}^{\mu}(x)$ are both greater than $\widetilde{A}_{\mu}$, we have $x_{n_{j}, m_{j}}^{\mu}>\widetilde{A}_{\mu}$ for all $j$.

If there is a first $j \in\{1, \ldots, k\}$ with $n_{j}=1$ (and thus $m_{j}=0$ ) then, since $\mu \geq 0$, by the second part of Remark 3.2, one has

$$
x_{n_{j}, m_{j}}^{\mu}=T_{1,0}^{\mu}\left(x_{n_{j-1}, m_{j-1}}^{\mu}\right)>T_{1,0}^{\mu}\left(\widetilde{A}_{\mu}\right) \geq \widetilde{R}_{\mu} .
$$

Remark 3.9 yields $x_{n_{k}, m_{k}}^{\mu}>\widetilde{R}_{\mu}$ and thus $x_{n_{k}, m_{k}}^{\mu} \notin V_{\varepsilon}$, which is a contradiction.

Hence we can assume that $n_{j}=0$ for all $j$ (thus $m_{j}=1$ for all $j$ ). Then $x_{n_{j}, m_{j}}^{\mu}$ is a decreasing sequence and $x_{n_{j}, m_{j}}^{\mu} \notin V_{\varepsilon}$ for all $j$, which is also a contradiction. The proof of (b) is now complete.

To prove (e) just observe that, by Lemma 3.4, if $x \in I_{1}$ then the return $(n, m)$ is of the form $(0, k)$ or $(1, n)$. In the first case, since $x \in I_{1}$ and by (E8), $\left(T_{0, k}^{\mu}\right)^{\prime}(x)<\lambda^{k} \varrho^{\mathrm{s}} \leq \bar{\varrho}<1$. In the second case, we have $\left(T_{1,0}^{\mu}\right)^{\prime}(x)<$ $\beta \varrho^{\mathrm{s}}=\bar{\varrho}<1$, ending the proof of (e).

The proof of (f) follows analogously by observing that if $x \in I_{3}$ then $(n, m)$ is of the form $(k, 0)$ or $(0,1)$ and using $(\mathrm{E} 8)$.

Corollary 3.11. For every $\mu \geq 0$ one has $\Omega^{\mu}=\Omega_{1}^{\mu} \cup \Omega_{\mu}^{3} \cup\left\{\widetilde{A}_{\mu}, \widetilde{R}_{\mu}\right\}$.

Proof. This follows immediately from Proposition 3.7(a)-(d).

3.4. Unfolding of cycles associated to $\widetilde{A}$ and $\widetilde{R}$. Consider the family $\mathcal{T}^{\mu}=\left(T_{n, m}^{\mu}\right)$ of maps. By condition $(\mathrm{E} 12), T_{1,0}^{0}(\widetilde{A})=\widetilde{R}$; this is expressed by saying that the family $\mathcal{T}^{\mu}$ has a cycle associated to $\widetilde{A}$ and $\widetilde{R}$ at $\mu=0$. Let

$$
U(\mu)=T_{1,0}^{\mu}\left(\widetilde{A}_{\mu}\right)-\widetilde{R}_{\mu},
$$

where $U(0)=0$. We say that the family $\mathcal{T}^{\mu}$ unfolds the cycle at $\mu=0$ with positive velocity if $U^{\prime}(0)>0$ (i.e., the points $T_{1,0}^{\mu}\left(\widetilde{A}_{\mu}\right)$ and $\widetilde{R}_{\mu}$ move with positive velocity which respect to each other).

LEMMA 3.12. The family $\mathcal{T}^{\mu}$ of maps unfolds the cycle associated to $\widetilde{A}$ and $\widetilde{R}$ at $\mu=0$ with positive velocity, that is, $U^{\prime}(0)>0$.

Proof. Observe that

$$
T_{1,0}^{\mu}(x)=\beta(\Pi(x)+1+\mu)=T_{1,0}^{0}(x)+\beta \mu, \quad T_{0,1}^{\mu}(x)=\lambda \Pi(x)+1+\mu .
$$


Thus

$$
U(\mu)=T_{1,0}^{\mu}\left(\widetilde{A}_{\mu}\right)-\widetilde{R}_{\mu}=T_{1,0}^{0}\left(\widetilde{A}_{\mu}\right)+\beta \mu-\widetilde{R}_{\mu}
$$

Then

$$
U^{\prime}(0)=\left(T_{1,0}^{0}\right)^{\prime}(\widetilde{A}) \frac{d \widetilde{A}_{\mu}}{d \mu}(0)+\beta-\frac{d \widetilde{R}_{\mu}}{d \mu}(0)=\beta \Pi^{\prime}(\widetilde{A}) \frac{d \widetilde{A}_{\mu}}{d \mu}(0)+\beta-\frac{d \widetilde{R}_{\mu}}{d \mu}(0) .
$$

From $\left(T_{0,1}^{0}\right)^{\prime}(\widetilde{A})=\lambda \Pi^{\prime}(\widetilde{A})=\lambda_{A}($ condition $(\mathrm{E} 9))$, it follows that $\Pi^{\prime}(\widetilde{A})=$ $\lambda_{A} / \lambda$. Since $\left(T_{1,0}^{0}\right)^{\prime}(\widetilde{A})=\beta \Pi^{\prime}(\widetilde{A})$, one has

$$
U^{\prime}(0)=\frac{\beta \lambda_{A}}{\lambda} \frac{d \widetilde{A}_{\mu}}{d \mu}(0)+\beta-\frac{d \widetilde{R}_{\mu}}{d \mu}(0) .
$$

Finally, if we observe that by (E9), $\widetilde{A}_{\mu} \simeq \lambda_{A} \widetilde{A}_{\mu}+b+\mu$ and $\widetilde{R}_{\mu} \simeq \beta_{R} \widetilde{R}_{\mu}+c+\mu$ for some $b$ and $c$, a straightforward calculation gives

$$
\frac{d \widetilde{A}_{\mu}}{d \mu}(0)=\frac{1}{1-\lambda_{A}} \quad \text { and } \quad \frac{d \widetilde{R}_{\mu}}{d \mu}(0)=\frac{1}{1-\beta_{R}} .
$$

Thus

$$
U^{\prime}(0)=\frac{\beta \lambda_{A}}{\lambda\left(1-\lambda_{A}\right)}+\beta-\frac{1}{1-\beta_{R}}>\beta>0 .
$$

\section{ONE-DIMENSIONAL MODEL FOR THE UNFOLDING OF PRIMARY CYCLES}

In this section we give a model for the unfolding of cycles associated to $\widetilde{A}$ and $\widetilde{R}$ and study the dynamics after the unfolding of such a cycle.

4.1. Primary cycles associated to $\widetilde{A}$ and $\widetilde{R}$. Consider $0<\lambda_{A}<1<$ $\beta_{R}$ as in condition (E9) and, as at the beginning of Section 3, the linear maps $x \mapsto \lambda_{A} x$ and $x \mapsto \beta_{R} x$, and their fundamental domains $\widetilde{J}=\left[-1,-\lambda_{A}\right]$ and $\widetilde{I}=\left[\beta_{R}^{-1}, 1\right]$. Consider also a strictly increasing differentiable map $\nabla$ from $\widetilde{I}$ to $\widetilde{J}$ with $\nabla(\widetilde{I})=\widetilde{J}$ having the following distortion property:

$$
\frac{1-\lambda_{A}}{2\left(1-\beta_{R}^{-1}\right)} \leq \nabla^{\prime}(x) \leq \frac{2\left(1-\lambda_{A}\right)}{1-\beta_{R}^{-1}} \quad \text { for all } x \in \widetilde{I} .
$$

In our applications we will have $\left(T_{0,1}^{0}\right)^{N_{3}}=\nabla$ (recall the distortion estimate in (E9)).

Consider differentiable monotone functions $S, U:[-1,1] \rightarrow \mathbb{R}$ with

$$
S(0)=U(0)=0, \quad S^{\prime}(0)=\kappa>0, \quad U^{\prime}(0)=\tau>0 .
$$

For small positive $\nu$ define $g(\nu)$ by

$$
S(-g(\nu))+U(\nu)=0 .
$$


Observe that, for small $\nu>0, g(\nu)$ is uniquely defined. Consider the families of maps

$$
S^{\nu}:[-g(\nu), 0] \rightarrow[0, U(\nu)], \quad S^{\nu}(\cdot)=S(\cdot)+U(\nu) .
$$

Finally, for small $\nu>0$ consider the parametrized family $\mathcal{L}^{\nu}=\left(L_{n, m}^{\nu}\right)$ of endomorphisms

$$
L_{n, m}^{\nu}: \widetilde{I} \rightarrow \widetilde{I}, \quad L_{n, m}^{\nu}(\cdot)=\beta_{R}^{n} \circ S^{\nu} \circ \lambda_{A}^{m} \circ \nabla(\cdot), \quad n, m \geq 0,
$$

defined for each pair $(n, m), n, m \geq 0$, from a (maximal) subinterval $\widetilde{K}_{n, m}^{\nu}$ of $\widetilde{I}$ into $\widetilde{I}$. As in the case of the sets $K_{n, m}^{\mu}$ of the family $\mathcal{T}^{\mu}$, in general, $\widetilde{K}_{n, m}^{\nu} \neq \widetilde{I}$ and there are pairs $(n, m)$ such that $\widetilde{K}_{n, m}^{\nu}=\emptyset$ (see for instance Remark 4.1).

We say that $\mathcal{L}^{\mu}=\left(L_{n, m}^{\nu}\right)$ is an iterated function system with a cycle at $\nu=0$ having transition map $\nabla$ and unfolding map $S^{\nu}$. For notational simplicity assume that $U(\nu)=\nu$, but the general case follows identically.
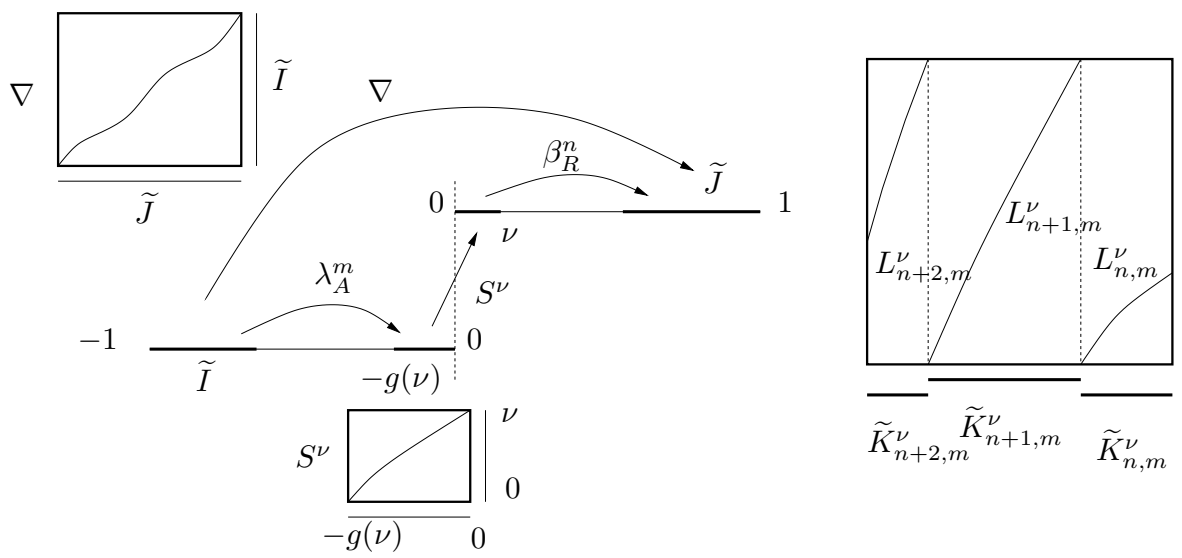

Fig. 6. The maps $L_{n, m}^{\nu}$

For each $\nu>0$ define $n(\nu)$ and $m(\nu)$ by the relations

$$
\lambda_{A} g(\nu) \leq \lambda_{A}^{m(\nu)}<g(\nu) \text { and } \beta_{R}^{-1} \nu \leq \beta_{R}^{-n(\nu)}<\nu .
$$

REMARK 4.1. Let $\nu>0$ be small and $m<m(\nu)-1$ or $n<n(\nu)-1$. Then $\widetilde{K}_{n, m}^{\nu}=\emptyset$.

Proof. Observe that if $m<m(\nu)-1$ then $\left.\left.\left.\lambda_{A}^{m} \nabla(x) \in\left[-\lambda_{A}^{m},-\lambda_{A}^{m+1}\right] \subset\right]-\infty,-\lambda_{A}^{m(\nu)-1}\right] \subset\right]-\infty,-g(\nu)[$ for all $x \in \widetilde{I}$. Thus $S^{\nu}\left(\lambda_{A}^{m} \nabla(x)\right)<0$ and $\beta_{R}^{k} S^{\nu}\left(\lambda_{A}^{m} \nabla(x)\right)<0$ for all $k \geq 0$. In particular, $\beta_{R}^{k} S^{\nu}\left(\lambda_{A}^{m} \nabla(x)\right) \notin \widetilde{I}$ for all $k \geq 0$. This ends the proof under the condition on $m$. The proof for $n$ is analogous and it is omitted. 
LEMMa 4.2. For every $\nu>0$ sufficiently small,

$$
\widetilde{I} \subset \bigcup_{j \geq n(\nu)} \widetilde{K}_{j, m(\nu)+1}^{\nu}
$$

Proof. By the definition of $m(\nu)$ in (4.7),

$$
\lambda_{A}^{m(\nu)+1} \nabla(\widetilde{I})=\lambda_{A}^{m(\nu)+1} \widetilde{J} \subset\left[-\lambda_{A} g(\nu), 0\right] .
$$

Therefore,

$$
S^{\nu}\left(\lambda_{A}^{m(\nu)+1} \widetilde{J}\right) \subset\left[S\left(-\lambda_{A} g(\nu)\right)+\nu, \nu\right]
$$

Since $S^{\prime}(0)=\kappa$, for a fixed small $\varepsilon$ there is $\nu_{0}$ such that, for every $\nu \in\left(0, \nu_{0}\right)$,

$$
\begin{gathered}
-(\kappa+\varepsilon) \lambda_{A} g(\nu)<S\left(-\lambda_{A} g(\nu)\right)<-(\kappa-\varepsilon) \lambda_{A} g(\nu), \\
\frac{\nu}{\kappa+\varepsilon}<g(\nu)<\frac{\nu}{\kappa-\varepsilon} .
\end{gathered}
$$

Write

$$
d^{+}(\varepsilon)=\frac{\kappa+\varepsilon}{\kappa-\varepsilon} \quad \text { and } \quad d^{-}(\varepsilon)=\frac{\kappa-\varepsilon}{\kappa+\varepsilon}, \quad \lim _{\varepsilon \rightarrow 0} d^{ \pm}(\varepsilon)=1 .
$$

Hence

$$
-d^{+}(\varepsilon) \lambda_{A} \nu<S\left(-\lambda_{A} g(\nu)\right)<-d^{-}(\varepsilon) \lambda_{A} \nu .
$$

Therefore, for small $\varepsilon$, that is, if $\nu \in\left(0, \nu_{0}\right)$ and $\nu_{0}$ is small enough,

$$
\left.\left.S^{\nu}\left(\lambda_{A}^{m(\nu)+1} \widetilde{J}\right) \subset\left[\left(1-d^{+}(\varepsilon) \lambda_{A}\right) \nu, \nu\right] \subset\right] 0, \nu\right] .
$$

Thus, by the definition of $n(\nu)$ in (4.7), for each $x \in \widetilde{I}$ there is $j(x) \geq 0$ such that

$$
\beta_{R}^{j(x)+n(\nu)} S^{\nu}\left(\lambda_{A}^{m(\nu)+1} \nabla(x)\right) \in \widetilde{I} .
$$

Hence $x \in \widetilde{K}_{j(x)+n(\nu), m(\nu)+1}^{\nu}$. Since $j(x) \geq 0$ this proves the lemma.

The definition of the sets $\widetilde{K}_{j, m(\nu)+1}^{\nu}$ gives immediately the following:

REMARK 4.3. For every $j$ and $k$ the set $\bigcup_{i=j}^{j+k} \widetilde{K}_{i, m(\nu)+1}^{\nu}$ is either empty, a point, or a closed interval.

Lemma 4.4. Consider $i \geq n(\nu)$ such that the sets $\widetilde{K}_{i-1, m(\nu)+1}^{\nu}, \widetilde{K}_{i, m(\nu)+1}^{\nu}$ and $\widetilde{K}_{i+1, m(\nu)+1}^{\nu}$ are all nonempty. Then $L_{i, m(\nu)+1}^{\nu}\left(\widetilde{K}_{i, m(\nu)+1}^{\nu}\right)=\widetilde{I}$.

Proof. For each $j=\{i-1, i, i+1\}$ let $l_{j}$ and $r_{j}, l_{j}<r_{j}$, be the extremes of $\widetilde{K}_{j, m(\nu)+1}^{\nu}$. Observe that $l_{j}=r_{j+1}$. The definition of the extremes of $\widetilde{K}_{i, m(\nu)+1}^{\nu}$ and the fact that $\widetilde{K}_{i-1, m(\nu)+1}^{\nu}$ and $\widetilde{K}_{i+1, m(\nu)+1}^{\nu}$ are both nonempty imply that $L_{i, m(\nu)+1)}^{\nu}\left(l_{i}\right)=\beta_{R}^{-1}$ and $L_{i, m(\nu)+1)}^{\nu}\left(r_{i}\right)=1$. This implies immediately the lemma. 
LEMMA 4.5. Consider $j$ such that $\widetilde{K}_{j, m(\nu)+1}^{\nu} \neq \emptyset$. Then $\left(L_{j, m(\nu)+1}^{\nu}\right)^{\prime}(x)>2 \xi>2 \quad$ for every $x \in \widetilde{K}_{j, m(\nu)+1}^{\nu}$, with $\xi>1$ as in (E9). (If $x$ is at the boundary of $\widetilde{K}_{j, m(\nu)+1}^{\nu}$ we consider the right or left derivative at $x$, according to the case.)

Proof. We first claim that

$$
\beta_{R}^{j} \geq \frac{1}{\left(1-\lambda_{A}^{2}\right) \nu \beta_{R}^{2}} .
$$

For that just observe that, by definition of $m(\nu)$ in $(4.7), \lambda_{A}^{m(\nu)+1} \widetilde{I} \subset$ ]$\left.-g(\nu),-\lambda_{A}^{2} g(\nu)\right]$. Thus, arguing as in the proof of Lemma 4.2 (recall (4.8)), one has

$$
\left.\left.S^{\nu}\left(\lambda_{A}^{m(\nu)+1} \nabla(\widetilde{I})\right) \subset\right] 0,\left(1-d^{-}(\varepsilon) \lambda_{A}^{2}\right) \nu\right]
$$

Since $\widetilde{K}_{j, m(\nu)+1}^{\nu} \neq \emptyset$ there is $x \in S^{\nu}\left(\lambda_{A}^{m(\nu)+1} \nabla(\widetilde{I})\right)$ with $\beta_{R}^{j}(x) \in\left[\beta_{R}^{-1}, 1\right]$. By (4.10),

$$
\beta_{R}^{j} \geq \frac{1}{\left(1-d^{-}(\varepsilon) \lambda_{A}^{2}\right) \nu \beta_{R}}>\frac{1}{\left(1-\lambda_{A}^{2}\right) \nu \beta_{R}^{2}}
$$

if $\varepsilon$ is small enough (i.e., $\nu$ is small). This ends the proof of (4.9).

The hypothesis on the derivative of $\nabla$ in (4.5), the estimates of $\lambda_{A}^{m(\nu)}$, $\beta_{R}^{n(\nu)}$, and $\beta_{R}^{j}$ in (4.7) and in (4.9), the expression of $L_{n, m}^{\mu}$ in (4.6), $S^{\prime}(0)=\kappa$, $g(\nu)>\nu /(\kappa+\varepsilon)$ if $\nu$ is small, $d^{-}(\varepsilon) \rightarrow 1$ as $\varepsilon \rightarrow 0$, and condition (E9) give immediately the following (for small $\varepsilon$ ):

$$
\begin{aligned}
\left(L_{j, m(\nu)+1}^{\nu}\right)^{\prime}(x) & \geq \beta_{R}^{j}(\kappa-\varepsilon) \lambda_{A}^{m(\nu)+1} \frac{1-\lambda_{A}}{2\left(1-\beta_{R}^{-1}\right)} \\
& \geq \frac{\lambda_{A}^{2} g(\nu)(\kappa-\varepsilon)\left(1-\lambda_{A}\right)}{2\left(1-\beta_{R}^{-1}\right)\left(1-\lambda_{A}^{2}\right) \nu \beta_{R}^{2}} \\
& \geq \frac{\lambda_{A}^{2}(\kappa-\varepsilon)\left(1-\lambda_{A}\right)}{2(\kappa+\varepsilon)\left(1-\beta_{R}^{-1}\right)\left(1-\lambda_{A}^{2}\right) \beta_{R}^{2}} \\
& \geq \frac{d^{-}(\varepsilon) \lambda_{A}^{2}}{2\left(1-\beta_{R}^{-1}\right)\left(1+\lambda_{A}\right) \beta_{R}}>2 \xi>2
\end{aligned}
$$

Proposition 4.6. For every small $\nu>0$ and every open subinterval $U$ of $\widetilde{I}$ there are a point $x \in U$, integers $k \geq 0$ and $m>0$, and a sequence $\left(n_{i}, m(\nu)+1\right)_{i=1}^{k}, n_{i} \geq n(\nu)$, of pairs such that

$$
S^{\nu}\left(\lambda_{A}^{m} \nabla\left(L_{n_{k}, m(\nu)+1}^{\nu} \circ L_{n_{2}+, m(\nu)+1}^{\nu} \circ L_{n_{1}, m(\nu)+1}^{\nu}(x)\right)\right)=0 .
$$

Before proving the proposition let us state the following corollary:

Corollary 4.7. For every $\nu \in\left(0, \nu_{0}\right)$ we have $\Omega\left(\mathcal{L}^{\nu}\right)=\widetilde{I}$. 
Proof. Just observe that, by Proposition 4.6, given any $x \in \widetilde{I}$ there are an interval $V$ arbitrarily close to $x$ and arbitrarily small, integers $k \geq 0$ and $m>0$, a sequence $\left(n_{i}, m(\nu)+1\right)_{i=1}^{k}, n_{i} \geq n(\nu)$, of pairs and large $j$ such that

$$
S^{\nu}\left(\lambda_{A}^{m} \nabla\left(L_{n_{k}, m(\nu)+1}^{\nu} \circ L_{n_{2}+, m(\nu)+1}^{\nu} \circ L_{n_{1}, m(\nu)+1}^{\nu}(V)\right)\right)=\beta_{R}^{-j} \widetilde{I} .
$$

This means that

$$
L_{j, m}^{\nu} \circ L_{n_{k}, m(\nu)+1}^{\nu} \circ L_{n_{2}+, m(\nu)+1}^{\nu} \circ L_{n_{1}, m(\nu)+1}^{\nu}(V)=\widetilde{I},
$$

which automatically implies the corollary.

Proof of Proposition 4.6. By (4.7), $g(\nu) \in\left[\lambda_{A}^{m(\nu)}, \lambda_{A}^{m(\nu)-1}\right]$. Thus, $-g(\nu)$ $\in \lambda_{A}^{m(\nu)} \widetilde{J} \cup \lambda_{A}^{m(\nu)-1} \widetilde{J}$ and there are $y \in \widetilde{I}$ and $m \in\{m(\nu)-1, m(\nu)\}$ such that

$$
S^{\nu}\left(\lambda_{A}^{m} \nabla(y)\right)=0 .
$$

Thus to prove the proposition it is enough to see the following:

Claim 4.8. There are a subinterval $U_{0}$ of $U$ and finite sequences $\left(n_{i}, m(\nu)+1\right)_{i=1}^{k}, n_{i} \geq n(\nu)$, of pairs and $\left(U_{i}\right)_{i=0}^{k}$ of subintervals of $\widetilde{I}$ such that

- $U_{i} \subset \widetilde{K}_{n_{i+1}, m(\nu)+1}^{\nu}$,

- $U_{i+1} \subset L_{n_{i+1}, m(\nu)+1}^{\nu}\left(U_{i}\right)$,

- $U_{k}=\widetilde{I}$.

Proof. Given a subinterval $V$ of $\widetilde{I}$ there are three possibilities:

(A) there is $n \geq n(\nu)$ such that $V \subset \widetilde{K}_{n, m(\nu)+1}^{\nu}$,

(B) there is $n \geq n(\nu)$ such that $V \subset \widetilde{K}_{n, m(\nu)+1}^{\nu} \cup \widetilde{K}_{n+1, m(\nu)+1}^{\nu}$,

(C) there is $n \geq n(\nu)$ with $\widetilde{K}_{n, m(\nu)+1}^{\nu} \subset V$ and $L_{n, m(\nu)+1}\left(\widetilde{K}_{n, m(\nu)+1}^{\nu}\right)=\widetilde{I}$.

To prove this assertion suppose, by contradiction, that $(\mathrm{A})$ and $(\mathrm{B})$ do not hold. By Lemma 4.2, there is $n \geq n(\nu)$ such that $\widetilde{K}_{n, m(\nu)+1}^{\nu} \cap V \neq \emptyset$. We take the minimum $n$ with this property. By hypothesis, $V$ is not contained in $\widetilde{K}_{n, m(\nu)+1}^{\nu} \cup \widetilde{K}_{n+1, m(\nu)+1}^{\nu}$. Thus the choice of $n$, Lemma 4.2 and Remark 4.3 imply that $\widetilde{K}_{n+2, m(\nu)+1}^{\nu} \neq \emptyset$ and $\widetilde{K}_{n+1, m(\nu)+1}^{\nu} \subset V$. Finally, Lemma 4.4 shows that

$$
L_{n+1, m(\nu)+1}^{\nu}\left(\widetilde{K}_{n+1, m(\nu)+1}^{\nu}\right)=\widetilde{I} .
$$

The assertion now follows on recalling that $\widetilde{K}_{n+1, m(\nu)+1}^{\nu} \subset V$.

We are now ready to prove the claim. If the initial interval $U$ satisfies $(\mathrm{C})$ we are done. Otherwise, in cases (A) and (B), to the interval $U$ we associate 
$n=n(U)$ as in the proof of the assertion above and write

$$
U=U^{-} \cup U^{+}, \quad U^{-}=U \cap \widetilde{K}_{n+1, m(\nu)+1}^{\nu}, \quad U^{+}=U \cap \widetilde{K}_{n, m(\nu)+1}^{\nu},
$$

where $U^{-}=\emptyset$ in case $(\mathrm{A})$. We define

$$
\widetilde{U}^{-}=L_{n+1, m(\nu)+1}\left(U^{-}\right), \quad \widetilde{U}^{+}=L_{n, m(\nu)+1}\left(U^{+}\right) .
$$

By Lemma 4.5,

$$
\left|\widetilde{U}^{ \pm}\right| \geq 2 \xi\left|U^{ \pm}\right|
$$

Since $U=U^{+} \cup U^{-}$, either $\left|U^{+}\right|$or $\left|U^{-}\right|$is greater than or equal to $|U| / 2$, thus

$$
\max \left\{\left|\widetilde{U}^{+}\right|,\left|\widetilde{U}^{-}\right|\right\} \geq \xi|U|, \quad \text { where } \xi>1 .
$$

If the maximum is $\left|\widetilde{U}^{+}\right|$we let $U_{0}=U^{+}$and $n_{1}=n$. Otherwise, we take $U_{0}=U^{-}$and $n_{1}=n+1$.

Take now

$$
U_{0}^{\prime}=L_{n_{1}, m(\nu)+1}^{\nu}\left(U_{0}\right), \quad \text { where } \quad\left|U_{0}^{\prime}\right| \geq \xi|U| .
$$

If $U_{0}^{\prime}$ satisfies (C) we are done. Otherwise, arguing as in the previous step, we get $n_{2}$ and a subinterval $U_{1}$ of $U_{0}^{\prime}$ such that $U_{1} \subset \widetilde{K}_{n_{2}, m(\nu)+1}^{\nu}$ and

$$
\left|U_{1}^{\prime}\right|>\xi\left|U_{0}^{\prime}\right|>\xi^{2}|U|, \quad \text { where } \quad U_{1}^{\prime}=L_{n_{2}, m(\nu)+1}^{\nu}\left(U_{1}\right) .
$$

We are now ready to end the proof of the claim, which follows inductively. Suppose we have defined $U_{i}, U_{i}^{\prime}$ and $n_{i}$ such that every $U_{i}$ satisfies (A) or (B) and $\left|U_{i}^{\prime}\right|>\xi^{i}|U|$, where $U_{i} \subset \widetilde{K}_{n_{i}, m(\nu)+1}^{\nu}$ and $U_{i}^{\prime}=L_{n_{i}, m(\nu)+1}^{\nu}\left(U_{i}\right)$. Since the size of the sets $\widetilde{K}_{n, m(\nu)+1}^{\nu}$ is bounded there is a first $k$ such that $U_{k}^{\prime}$ satisfies (C), and we are done.

As mentioned, the proposition is a direct consequence of the claim.

Let us make a remark about the results on the iterated function system $\left(L_{n, m}^{\nu}\right)$.

REMARK 4.9. The constructions in Section 4 also work if the eigenvalues $\lambda_{A}(\nu)$ and $\beta_{R}(\nu)$ depend continuously on $\nu$, thus the reference fundamental domains $\widetilde{I}_{\nu}=\left[\beta_{R}(\nu)^{-1}, 1\right]$ and $\widetilde{J}_{\nu}=\left[-1,-\lambda_{A}(\nu)\right]$ also depend on $\nu$. In this case one considers transitions $\nabla^{\nu}$ depending continuously in the $C^{1}$-topology on the parameter $\nu$ with $\nabla_{\nu}(\widetilde{I}(\nu))=\widetilde{J}(\nu)$ and satisfying the distortion property in (4.5) for every small $\nu$.

\subsection{The dynamics after the unfolding of the cycle associated to} $\widetilde{A}$ and $\widetilde{R}$. Bearing in mind Remark 4.9 we now construct an appropriate iterated function system $\mathcal{F}^{\mu}=\left(F_{n, m}^{\mu}\right)$ which describes the dynamics in the unfolding of the cycle associated to $\widetilde{A}$ and $\widetilde{R}$ for $\mathcal{T}^{\mu}$. 
Given $\mu$ close to 0 let $\widetilde{R}_{\mu}$ and $\widetilde{A}_{\mu}$ be the continuations for $T_{0,1}^{\mu}$ of the fixed points $\widetilde{R}$ and $\widetilde{A}$ of $T_{0,1}^{0}$. Denote by $\beta_{R}(\mu)$ and $\lambda_{A}(\mu)$ the corresponding eigenvalues. Consider continuations $\widetilde{C}_{\mu}$ and $\widetilde{D}_{\mu}$ of the points $\widetilde{C}$ and $\widetilde{D}$ in (E9) such that

$$
\left(T_{0,1}^{\mu}\right)^{N_{3}}\left(\left[\beta_{R}(\mu)^{-1} \widetilde{D}_{\mu}, \widetilde{D}_{\mu}\right]\right)=\left[\lambda_{A}(\mu) \widetilde{C}_{\mu}, \widetilde{C}_{\mu}\right] .
$$

For each $\mu \in\left(-\mu_{0}, \mu_{0}\right)$ with $|\mu|$ small, consider the affine bijective maps

$$
\psi_{R}^{\mu}:\left[\widetilde{D}_{\mu}, \widetilde{R}_{\mu}\right] \rightarrow[0,1] \text { and } \psi_{A}^{\mu}:\left[\widetilde{A}_{\mu}, \widetilde{C}_{\mu}\right] \rightarrow[-1,0]
$$

with $\psi_{A}^{\mu}\left(\widetilde{A}_{\mu}\right)=0, \psi_{A}^{\mu}\left(\widetilde{C}_{\mu}\right)=-1, \psi_{R}^{\mu}\left(\widetilde{D}_{\mu}\right)=1$, and $\psi_{\mu}^{R}\left(\widetilde{R}_{\mu}\right)=0$.

By Remark 3.2 , for small $\mu<0$ there is a unique $\left.\widetilde{E}_{\mu} \in\right] \widetilde{A}_{\mu}, \widetilde{C}_{\mu}$ [ with $T_{1,0}^{\mu}\left(\widetilde{E}_{\mu}\right)=\widetilde{R}_{\mu}$, where $\widetilde{E}_{\mu} \rightarrow \widetilde{A}_{\mu}$ as $\mu \rightarrow 0^{-}$.

The iterated function system $\mathcal{F}^{\mu}=\left(F_{n, m}^{\mu}\right), \mu<0$, is defined as follows. For each small $\mu<0$ consider the fundamental domains $\widetilde{I}_{\mu}=\left[\beta_{R}(\mu)^{-1}, 1\right]$ and $\widetilde{J}_{\mu}=\left[-1,-\lambda_{A}(\mu)\right]$.

- The transition map of $\mathcal{F}^{\mu}$ is the map $\nabla^{\mu}$ defined by

$$
\nabla^{\mu}: \widetilde{I}_{\mu} \rightarrow \widetilde{J}_{\mu}, \quad \nabla^{\mu}(\cdot)=\psi_{A}^{\mu} \circ\left(\left(T_{0,1}^{\mu}\right)^{N_{3}}\right) \circ\left(\psi_{R}^{\mu}\right)^{-1}(\cdot) .
$$

Observe that by construction $\nabla^{\mu}\left(\widetilde{I}_{\mu}\right)=\widetilde{J}_{\mu}$ and that $\nabla^{\mu}$ has the distortion property (4.5), i.e.,

$$
\frac{1-\lambda_{A}}{1-\beta_{R}} \leq D\left(\nabla^{\mu}\right)(x) \leq 2 \frac{1-\lambda_{A}}{1-\beta_{R}}
$$

For the last assertion just observe that, by condition (E9), $\left(T_{0,1}^{\mu}\right)^{N_{3}}$ has this property for points in $\left[\beta_{R}(\mu)^{-1}, \widetilde{D}_{\mu}\right]$ and that for every $x \in[-1,0]$ and $y \in[0,1]$,

$$
\lim _{\mu \rightarrow 0^{-}}\left(\psi_{A}^{\mu}\right)^{\prime}(x)\left(\left(\psi_{R}^{\mu}\right)^{-1}\right)^{\prime}(y)=\lim _{\mu \rightarrow 0^{-}} \frac{\widetilde{C}_{\mu}-\widetilde{A}_{\mu}}{\widetilde{R}_{\mu}-\widetilde{D}_{\mu}}=\frac{\widetilde{C}-\widetilde{A}}{\widetilde{R}-\widetilde{D}}=1 ;
$$

for the last equality recall (E9).

- For $\mu<0$ close to 0 let $-g(\mu)=\psi_{A}^{\mu}\left(\widetilde{E}_{\mu}\right)$. The unfolding map (defined for negative $\mu$ ) of $\mathcal{F}^{\mu}$ is

$$
V^{\mu}:[-g(\mu), 0] \rightarrow[0,1], \quad V^{\mu}(x)=\left(\psi_{R}^{\mu}\right)^{-1} \circ T_{1,0}^{\mu} \circ \psi_{A}^{\mu}(x) .
$$

Finally, for small negative $\mu$, the system $\mathcal{F}^{\mu}$ is defined by

$$
F_{n, m}^{\mu}: \widetilde{I}_{\mu} \rightarrow \widetilde{I}_{\mu}, \quad F_{n, m}^{\mu}(\cdot)=\beta_{R}(\mu)^{n} \circ V^{\mu} \circ \lambda_{A}(\mu)^{m} \circ \nabla^{\mu}(\cdot), \quad n, m \geq 0 .
$$

REMARK 4.10. Observe that, up to a change of coordinates, the system $\mathcal{F}^{\mu}$ only involves iterations under $T_{1,0}^{\mu}$ and $T_{0,1}^{\mu}$ and points in $I_{2}$.

It is now immediate to check that we can repeat the arguments and the constructions of Section 4 using the map $V^{\mu}$ instead of $S^{\nu}$. Thus by 
Remark 4.10, Proposition 4.6 and Corollary 4.7 we immediately have the following results for the system $\mathcal{F}^{\mu}$ :

Proposition 4.11. For every small $\mu>0$ and every open subinterval $U$ of $I_{2}$ there are a point $x \in U$, integers $k \geq 0$ and $m>0$, and a sequence $\left(n_{i}, m(\mu)+1\right)_{i=1}^{k}, n_{i} \geq n(\mu)$, of pairs such that

$$
V^{\mu}\left(\lambda_{A}(\mu)^{m} \nabla^{\mu}\left(F_{n_{k}, m(\mu)+1}^{\mu} \circ F_{n_{2}, m(\mu)+1}^{\mu} \circ F_{n_{1}, m(\mu)+1}^{\mu}(x)\right)\right)=0 .
$$

COROllary 4.12. For every $\mu \in\left(-\mu_{0}, 0\right)$ we have $\Omega_{2}^{\mu}=I_{2}$.

We end this section with a remark that we will use in Section 9. We define the inverse iterated function system $\widetilde{\mathcal{F}}^{\mu}$ of $\mathcal{F}^{\mu}$ as follows. For each small $\mu$ consider the map

$$
\Upsilon^{\mu}=\left(\nabla^{\mu}\right)^{-1}=\left(T_{0,1}^{\mu}\right)^{-N_{3}}: \widetilde{J}_{\mu} \rightarrow \widetilde{I}_{\mu} .
$$

Then $\Upsilon^{\mu}$ is the transition map of the inverse system and, by (E9), it has the distortion property (4.5).

The unfolding map of $\widetilde{\mathcal{F}}^{\mu}$ is obtained as follows. For each $\mu \in\left(-\mu_{0}, \mu_{0}\right)$ with $|\mu|$ small, consider the affine bijective maps

$$
\vartheta_{R}^{\mu}:\left[\widetilde{D}_{\mu}, \widetilde{R}_{\mu}\right] \rightarrow[-1,0] \text { and } \vartheta_{A}^{\mu}:\left[\widetilde{A}_{\mu}, \widetilde{C}_{\mu}\right] \rightarrow[0,1]
$$

with $\vartheta_{A}^{\mu}\left(\widetilde{A}_{\mu}\right)=0, \vartheta_{A}^{\mu}\left(\widetilde{C}_{\mu}\right)=1, \vartheta_{R}^{\mu}\left(\widetilde{D}_{\mu}\right)=-1$, and $\vartheta_{R}^{\mu}\left(\widetilde{R}_{\mu}\right)=0$.

For small $\mu<0$ let $T_{1,0}^{\mu}\left(A_{\mu}\right)=\widetilde{E}_{\mu}^{\prime}$. Write $-\widetilde{g}(\mu)=\vartheta_{R}^{\mu}\left(\widetilde{E}_{\mu}^{\prime}\right)$. The unfolding map (defined for negative $\mu$ ) is

$$
\widetilde{V}^{\mu}:[-\widetilde{g}(\mu), 0] \rightarrow[0,1], \quad \widetilde{V}^{\mu}(x)=\left(\vartheta_{A}^{\mu}\right)^{-1} \circ\left(T_{1,0}^{\mu}\right)^{-1} \circ \vartheta_{R}^{\mu}(x) .
$$

Finally, the inverse system $\widetilde{\mathcal{F}}^{\mu}$ is defined for negative $\mu$ by

$$
\widetilde{F}_{n, m}^{\mu}: \widetilde{J}_{\mu} \rightarrow \widetilde{J}_{\mu}, \quad \widetilde{F}_{n, m}^{\mu}(\cdot)=\lambda_{A}(\mu)^{-n} \circ \widetilde{V}^{\mu} \circ \beta_{R}(\mu)^{-m} \circ \Upsilon^{\mu}(\cdot), \quad n, m \geq 0 .
$$

REMark 4.13. Proposition 4.11 holds for the system $\widetilde{\mathcal{F}}^{\mu}$.

\section{CHOICE OF PARAMETERS}

In this section we construct sequences of intervals $L_{k}=\left[a_{k}, b_{k}\right]$ and reparameterizations $\mu_{k}: L_{k} \rightarrow\left[-\mu_{0} / 2, \mu_{0} / 2\right]$ such that the maps $G_{m, n}^{t}$ defined in (2.1) of Section 2.2 are arbitrarily $C^{0}$-close to the model maps $T_{n, m}^{\mu_{k}(t)}$ for all $t \in L_{k}$. In fact, for fixed $n_{0}$ and $m_{0}$, we get $C^{1}$-proximity if $n \in\left\{0, \ldots, n_{0}\right\}$ and $m \in\left\{0, \ldots, m_{0}\right\}$. Observe that in our one-dimensional model the eigenvalues $\lambda$ and $\beta$ of $P$ and $Q$ do not depend on $t$. Having in mind that we want to prove the results for families close to our model, in this section we assume that these eigenvalues (denoted by $\lambda(t)$ and $\beta(t)$ ) are $C^{1}$-functions. 
LEMmA 5.1. There is $N_{0} \in \mathbb{N}$ such that for every $n \geq N_{0}$ there exists $\bar{t}_{n}>0$ satisfying

$$
\lambda\left(\bar{t}_{n}\right)^{n}=\bar{t}_{n}
$$

Moreover, $\lim _{n \rightarrow \infty} \bar{t}_{n}=0$.

Proof. As $\lambda(t)$ is a continuous function and $\lambda=\lambda(0) \in] 0,1[$, there is $t_{0}>0$ such that $\left.\lambda(t) \in\right] 0,1\left[\right.$ for all $t \in\left[0, t_{0}\right]$. Thus there exists $N_{0} \in \mathbb{N}$ such that $\lambda\left(t_{0}\right)^{n}<\lambda\left(t_{0}\right)^{N_{0}}<t_{0}$ for every $n \geq N_{0}$. Now the lemma follows from the mean value theorem by just observing that $0<\lambda(0)^{n}$. The second assertion is trivial.

To get the positive density at zero of the intervals $L_{n}$ we need to estimate the frequency of the $\bar{t}_{n}$. This is done in the next lemma.

LEMMA 5.2. The sequence $\left(\bar{t}_{n}\right)_{n}$ of parameters in Lemma 5.1 satisfies

$$
\lim _{n \rightarrow \infty} \bar{t}_{n+1} / \bar{t}_{n}=\lambda(0) \text {. }
$$

Proof. First observe that

$$
\begin{aligned}
\bar{t}_{n}-\bar{t}_{n+1} & =\lambda\left(\bar{t}_{n}\right)^{n}-\lambda\left(\bar{t}_{n+1}\right)^{n+1} \\
& =(n+1) \lambda(\zeta)^{n} \lambda^{\prime}(\zeta)\left(\bar{t}_{n}-\bar{t}_{n+1}\right)+\bar{t}_{n}\left(1-\lambda\left(\bar{t}_{n}\right)\right)
\end{aligned}
$$

for some $\zeta \in] \bar{t}_{n+1}, \bar{t}_{n}[$. Therefore,

$$
\bar{t}_{n}\left(\lambda\left(\bar{t}_{n}\right)-(n+1) \lambda(\zeta)^{n} \lambda^{\prime}(\zeta)\right)=\bar{t}_{n+1}\left(1-(n+1) \lambda(\zeta)^{n} \lambda^{\prime}(\zeta)\right) .
$$

Hence,

$$
\frac{\bar{t}_{n+1}}{\bar{t}_{n}}=\frac{\lambda\left(\bar{t}_{n}\right)-(n+1) \lambda(\zeta)^{n} \lambda^{\prime}(\zeta)}{1-(n+1) \lambda(\zeta)^{n} \lambda^{\prime}(\zeta)} \rightarrow \lambda(0) .
$$

Now we define $\nu=\log (\beta(0)) / \log \left(\lambda(0)^{-1}\right)$. First assume that $\nu$ is irrational. Then for any fixed $\varepsilon>0$ there are sequences $n_{k} \in \mathbb{N}$ and $m_{k} \in \mathbb{Z}$ such that $n_{k} \rightarrow+\infty$ and $m_{k} \rightarrow-\infty$ and for every $k$ one has

$$
n_{k} \nu+m_{k} \in[0, \varepsilon[\text {. }
$$

LEMmA 5.3. Given $\varepsilon>0$ there is $N_{\varepsilon} \in \mathbb{N}$ such that the sequences $n_{k}$ and $m_{k}$ in (5.1) can be chosen to satisfy

$$
0<n_{k+1}-n_{k} \leq N_{\varepsilon} \quad \text { and } \quad\left|m_{k+1}-m_{k}\right| \leq N_{\varepsilon} \quad \text { for every } k \in \mathbb{N} \text {. }
$$

Proof. Observe that $n_{k+1}-n_{k}$ is uniformly bounded if and only if $\left|m_{k+1}-m_{k}\right|$ is. Thus it is enough to prove the result for the sequence $\left(n_{k}\right)_{k}$. For that choose $\bar{n}_{1}$ and $\bar{n}_{2}$ in $\mathbb{N}$ such that

$$
\bar{n}_{1} \nu \in\left[0, \varepsilon / 2\left[\bmod \mathbb{Z} \text { and } \bar{n}_{2} \nu \in\right]-\varepsilon / 2,0\right] \bmod \mathbb{Z}
$$

and let $N_{\varepsilon}=\max \left\{\bar{n}_{1}, \bar{n}_{2}\right\}$. We argue inductively: put $n_{1}=\bar{n}_{1}$ and suppose that $n_{1}, \ldots, n_{k}$ are defined satisfying

$$
0<n_{i+1}-n_{i} \leq N_{\varepsilon} \quad \text { and } \quad n_{i} \nu \in[0, \varepsilon[\bmod \mathbb{Z} .
$$


We now define

$$
n_{k+1}= \begin{cases}n_{k}+\bar{n}_{1} & \text { if } n_{k} \nu \in[0, \varepsilon / 2[\bmod \mathbb{Z} \\ n_{k}+\bar{n}_{2} & \text { if } n_{k} \nu \in[\varepsilon / 2, \varepsilon[\bmod \mathbb{Z} .\end{cases}
$$

In both cases, we have $n_{k+1} \nu \in\left[0, \varepsilon\left[\bmod \mathbb{Z}\right.\right.$ and $n_{k+1}-n_{k} \leq N_{\varepsilon}$, ending the proof of the lemma.

By Lemma 5.1, for each $m_{k}$ there is $t_{k}=\bar{t}_{-m_{k}}$ such that $\lambda\left(t_{k}\right)^{-m_{k}}=t_{k}$. From Lemmas 5.2 and 5.3 for every large $k$ we get

$$
\begin{aligned}
\frac{t_{k+1}}{t_{k}} & =\frac{\bar{t}_{-m_{k+1}}}{\bar{t}_{-m_{k+1}-1}} \cdot \frac{\bar{t}_{-m_{k+1}-1}}{\bar{t}_{-m_{k+1}-2}} \ldots \frac{\bar{t}_{-m_{k}+1}}{\bar{t}_{-m_{k}}} \\
& \simeq \lambda(0)^{m_{k}-m_{k+1}} \geq \lambda(0)^{N_{\varepsilon}},
\end{aligned}
$$

where $N_{\varepsilon}$ depends only on $\varepsilon$ (see Lemma 5.3).

When $\nu$ is rational we can also get sequences $n_{k}$ and $m_{k}$ as above:

REMARK 5.4. If $\nu=p / q$, where $p, q \in \mathbb{N}$ and $(p, q)=1$, then we let $n_{k}=k q$ and $m_{k}=-k p$, and define $t_{k}$ by the condition $\lambda\left(t_{k}\right)^{k p}=t_{k}$. In this case Lemma 5.3 and the estimate in (5.2) hold with $N_{\varepsilon}=q$.

From now on we assume that $\nu \notin \mathbb{Q}$, but the rational case can be handled in exactly the same way.

The parameters $t_{k}$ were chosen to satisfy $\lambda\left(t_{k}\right)^{-m_{k}}=t_{k}$. In the next result we get estimates for the values of the $\beta\left(t_{k}\right)^{-n_{k}}$. These estimates will play a key role in getting an accurate control of the derivative of returns in the central direction (as mentioned in Section 3.1, we need $\lambda\left(t_{k}\right)^{-m_{k}} \beta\left(t_{k}\right)^{n_{k}} \simeq 1$, see (5.12)).

Actually, we need to get estimates of $\lambda(t)^{-m_{k}}$ and $\beta(t)^{-n_{k}}$ for every $t$ in a scaled interval $L_{k}^{*}$ containing $t_{k}$, i.e., $\left|L_{k}^{*}\right| t_{k}^{-1}$ does not depend on $k$. These estimates are in (5.7) below. Finally, in (5.10) we describe exactly the intervals $L_{k}^{*}$.

Lemma 5.5. For every large $k$ one has

$$
\beta\left(t_{k}\right)^{-n_{k}} \in\left[\left(\lambda(0)^{\varepsilon}\left(1-D_{k}\right)+C_{k}\right) t_{k},\left(1-D_{k}+C_{k}\right) t_{k}\right],
$$

where the constants $C_{k}$ and $D_{k}$ satisfy $\lim _{k \rightarrow \infty} C_{k}=\lim _{k \rightarrow \infty} D_{k}=0$.

Proof. Since by definition $n_{k} \nu+m_{k} \in\left[0, \varepsilon\left[\right.\right.$ and $\beta(0)=\lambda(0)^{-\nu}$, we have

$$
\left.\left.\left.\left.\lambda(0)^{n_{k} \nu+m_{k}} \in\right] \lambda(0)^{\varepsilon}, 1\right], \quad \beta(0)^{-n_{k}} \in\right] \lambda(0)^{\varepsilon-m_{k}}, \lambda(0)^{-m_{k}}\right] .
$$

Now there is $\zeta \in] 0, t_{k}[$ such that

$$
\beta\left(t_{k}\right)^{-n_{k}}-\beta(0)^{-n_{k}}=C_{k} t_{k}, \quad C_{k}=-n_{k} \beta(\zeta)^{-n_{k}-1} \beta^{\prime}(\zeta) .
$$

Obviously, $\lim _{k \rightarrow \infty} C_{k}=0$. From (5.3) and (5.4) we get

$$
\left.\left.\beta\left(t_{k}\right)^{-n_{k}} \in\right] \lambda(0)^{\varepsilon-m_{k}}+C_{k} t_{k}, \lambda(0)^{-m_{k}}+C_{k} t_{k}\right] .
$$


Similarly, there is $\xi \in] 0, t_{k}[$ such that

$$
\lambda\left(t_{k}\right)^{-m_{k}}-\lambda(0)^{-m_{k}}=D_{k} t_{k}, \quad D_{k}=-m_{k} \lambda(\xi)^{-m_{k}-1} \lambda^{\prime}(\xi),
$$

and $\lim _{k \rightarrow \infty} D_{k}=0$. Recalling that by definition $\lambda\left(t_{k}\right)^{-m_{k}}=t_{k}$, we get $\lambda(0)^{-m_{k}}=t_{k}\left(1-D_{k}\right)$. The result now follows from (5.5).

Now we get estimates for $\beta(t)^{-n_{k}}$ and $\lambda(t)^{-m_{k}}$ for $t$ in a scaled interval $L_{k}^{*}$ around $t_{k}$. Writing $t=t_{k}(1+\varrho)$, as in the proof of Lemma 5.5, we have

$$
\begin{aligned}
\lambda\left(t_{k}(1+\varrho)\right)^{-m_{k}} & =\left(1+D_{k} \varrho\right) t_{k}, \\
\beta\left(t_{k}(1+\varrho)\right)^{-n_{k}} & =C_{k} \varrho t_{k}+\beta\left(t_{k}\right)^{-n_{k}},
\end{aligned}
$$

where $D_{k}$ and $C_{k}$ are not exactly the same as before (the derivatives of $\lambda(t)$ and $\beta(t)$ are calculated at different points of the parameter space), but for simplicity let us use the same notation.

We now determine an appropriate size for $\varrho$; for that we take small $s=s(\varepsilon)>0$ (to be determined exactly later, see also conditions (5.11) and (5.13)) and impose the following conditions:

$$
\begin{aligned}
& \left.\lambda\left(t_{k}(1+\varrho)\right)^{-m_{k}} \in\right](1-s) t_{k}(1+\varrho),(1+s) t_{k}(1+\varrho)[, \\
& \left.\beta\left(t_{k}(1+\varrho)\right)^{-n_{k}} \in\right](1-s) t_{k}(1+\varrho),(1+s) t_{k}(1+\varrho)[.
\end{aligned}
$$

Using (5.6) we see that this condition is satisfied by $\lambda\left(t_{k}(1+\varrho)\right)^{-m_{k}}$ if

$$
\frac{-s}{1+s-D_{k}}<\varrho<\frac{s}{1-s-D_{k}} .
$$

To assure that $\beta\left(t_{k}(1+\varrho)\right)^{-n_{k}}$ also satisfies (5.7) we use the second part of (5.6) and Lemma 5.5 to get

$$
\frac{\beta\left(t_{k}(1+\varrho)\right)^{-n_{k}}}{t_{k}} \in\left[\lambda(0)^{\varepsilon}\left(1-D_{k}\right)+C_{k}(1+\varrho), 1-D_{k}+C_{k}(1+\varrho)\right] .
$$

Thus it is enough to have

$$
\lambda(0)^{\varepsilon}\left(1-D_{k}\right)+C_{k}+C_{k} \varrho \geq(1-s)(1+\varrho)
$$

and

$$
1-D_{k}+C_{k}+C_{k} \varrho \leq(1+s)(1+\varrho) .
$$

Now these inequalities are satisfied if

$$
-1+\frac{1-D_{k}}{1+s-C_{k}} \leq \varrho \leq-1+\frac{\lambda(0)^{\varepsilon}\left(1-D_{k}\right)}{1-s-C_{k}} .
$$

As $C_{k}$ and $D_{k}$ tend to zero as $k \rightarrow \infty$, it follows that if

$$
\varrho \in]-1+\frac{1}{1+s},-1+\frac{\lambda(0)^{\varepsilon}}{1-s}[
$$


then $\beta\left(t_{k}(1+\varrho)\right)^{-n_{k}}$ satisfies (5.7) for every $k$ large enough. Hence (5.7) holds for every $t$ in the interval $L_{k}^{*}$ (large $k$ ) defined by

$$
\left.L_{k}^{*}=\right] t_{k}^{-}, t_{k}^{+}[=] \frac{1}{1+s} t_{k}, \frac{\lambda(0)^{\varepsilon}}{1-s} t_{k}[.
$$

We take

$$
s=s(\varepsilon)=2\left(\lambda(0)^{-\varepsilon}-1\right)>\lambda(0)^{-\varepsilon}-1>1-\lambda(0)^{\varepsilon},
$$

which, in particular, assures that $t_{k}$ belongs to the interior of $L_{k}^{*}$.

Observe that with this choice of $s$ (thus of $\varrho$ ) we have

$$
\frac{\left|L_{k}^{*}\right|}{t_{k}}=\frac{\left(\lambda(0)^{\varepsilon}-1\right)+s\left(\lambda(0)^{\varepsilon}+1\right)}{1-s^{2}} \geq \frac{s}{1-s^{2}} \lambda(0)^{\varepsilon},
$$

i.e., the $L_{k}^{*}$ are scaled intervals.

We now obtain some estimates on the products $\Delta_{k}(t)=\beta(t)^{n_{k}} \lambda(t)^{-m_{k}}$ for $t \in L_{k}^{*}$, which will be used for bounding the derivative of the returns in the central direction. First, from (5.7) we deduce that, for all $t \in L_{k}^{*}$,

$$
\frac{1-s}{1+s}<\Delta_{k}(t)=\beta(t)^{n_{k}} \lambda(t)^{-m_{k}}<\frac{1+s}{1-s} .
$$

We take $s$ small enough (that is, $\varepsilon$ close to zero) satisfying

$$
\varrho \frac{1-s}{1+s}>1 \text { and } \bar{\varrho} \frac{1+s}{1-s}<1,
$$

where $\varrho=\lambda \varrho^{\mathrm{u}}>1$ and $\bar{\varrho}=\beta \varrho^{\mathrm{s}}<1$ (see $(\mathrm{E} 8)$ for the definitions of $\varrho^{\mathrm{u}}$ and $\varrho^{\mathrm{s}}$ ).

Now, from (5.8), writing $t \in L_{k}^{*}$ in the form $t_{k}(1+\varrho)$ for some appropriate $\varrho$, we get $\xi_{k}^{ \pm} \rightarrow 1$ as $k \rightarrow \infty$ such that

$$
\beta(t)^{-n_{k}} \in\left[\lambda(0)^{\varepsilon} \xi_{k}^{-} t_{k}, \xi_{k}^{+} t_{k}\right] .
$$

Thus

$$
\frac{t}{t_{k} \xi_{k}^{+}} \leq \beta(t)^{n_{k}} t=1+\mu_{k}(t) \leq \frac{t}{\lambda(0)^{\varepsilon} t_{k} \xi_{k}^{-}} .
$$

For the extreme $t_{k}^{+}=\lambda(0)^{\varepsilon} t_{k} /(1-s)$ of the interval $L_{k}^{*}$ the previous equation gives

$$
\beta\left(t_{k}^{+}\right)^{n_{k}} t_{k}^{+} \geq \frac{\lambda(0)^{\varepsilon}}{(1-s) \xi_{k}^{+}}=1+\frac{(s-1) \xi_{k}^{+}+\lambda(0)^{\varepsilon}}{(1-s) \xi_{k}^{+}} \geq 1+\mu_{1},
$$

where $\mu_{1}$ is given by

$$
\mu_{1}=\frac{s-1+\lambda(0)^{\varepsilon}}{2(1-s)}
$$

Observe that condition (5.11) assures that $\mu_{1}>0$. 
Similarly, for $t_{k}^{-}=t_{k} /(1+s)$ we get

$$
\beta\left(t_{k}^{-}\right)^{n_{k}} t_{k}^{-} \leq \frac{1}{\lambda(0)^{\varepsilon}(1+s) \xi_{k}^{-}} \leq 1+\mu_{2}
$$

where

$$
\mu_{2}=\frac{1-(1+s) \lambda(0)^{\varepsilon}}{2 \lambda(0)^{\varepsilon}(1+s)}
$$

Observe that $\mu_{2}<0$ is equivalent to $s>\lambda(0)^{-\varepsilon}-1$, which again is guaranteed by (5.11).

It is clear that $\mu_{1}$ and $\mu_{2}$ go to zero as $\varepsilon$ (and thus $s=s(\varepsilon)$ ) tends to zero. Moreover, a straightforward calculation gives

$$
\lim _{\varepsilon \rightarrow 0} \frac{\mu_{1}}{-\mu_{2}}=1 \text {. }
$$

We now take

$$
\mu_{0}=\min \left\{\mu_{1},-\mu_{2}\right\},
$$

and observe that if $\varepsilon$ is small then

$$
\left[1-\mu_{0}, 1+\mu_{0}\right] \subset I_{1} .
$$

Lemma 5.6. There are constants $\kappa_{1}$ and $\kappa_{2}, 0<\kappa_{1}<\kappa_{2}$, such that for every $k$ large enough one has

$$
y \mu_{k}^{\prime}(w) \in\left[\kappa_{1}, \kappa_{2}\right] \quad \text { for every } y, w \in L_{k}^{*} .
$$

In particular,

$$
0<\kappa_{1} / t_{k}^{+} \leq \mu_{k}^{\prime}(w) \leq \kappa_{2} / t_{k}^{-} \quad \text { for all } w \in L_{k}^{*} .
$$

Proof. Observe that

$$
y \mu_{k}^{\prime}(w)=y\left(n_{k} \beta(w)^{n_{k}-1} \beta^{\prime}(w) w+\beta(w)^{n_{k}}\right) .
$$

We first claim that

$$
y n_{k} \beta(w)^{n_{k}-1} \beta^{\prime}(w) w \rightarrow 0 \quad \text { as } k \rightarrow \infty .
$$

In fact, (5.7) implies that this product is of order $t_{k} n_{k}$. Now our assertion follows using the fact that $\lambda\left(t_{k}\right)^{-m_{k}}=t_{k}$ and $n_{k} \simeq-m_{k}$ (see (5.1)).

Finally, using again (5.7) and (5.10) one gets strictly positive constants $\kappa_{1}^{\prime}$ and $\kappa_{2}^{\prime}$ (independent of large $k$ ) such that

$$
\kappa_{1}^{\prime}<y \beta(w)^{n_{k}}<\kappa_{2}^{\prime} .
$$

Now the lemma is a direct consequence of (5.19)-(5.21).

The next lemma follows immediately from the previous one.

LEMma 5.7. There is $v>0$ such that for every $k$ large enough there is a subinterval $L_{k}=\left[a_{k}, b_{k}\right]$ of $L_{k}^{*}$ such that

- $\mu_{k}\left(a_{k}\right)=-\mu_{0} / 2$ and $\mu_{k}\left(b_{k}\right)=\mu_{0} / 2$, 
- $\beta(t)^{n_{k}} t=1+\mu_{k}(t)$ and $\mu_{k}(t) \in\left[-\mu_{0} / 2, \mu_{0} / 2\right]$ for every $t \in L_{k}$; in particular, by (5.18), $\beta(t)^{n_{k}} t$ belongs to the interior of $I_{1}$,

- $\left|L_{k}\right| / t_{k}>v$.

The intervals $L_{k}$ will be the intervals in the parameter space referred to in the statement of the theorem. The third item in the lemma and (5.2) give immediately the positive relative density at the bifurcation value of the intervals $L_{k}$.

By Lemmas 5.6 and 5.7, for every $k>0$ large enough the inverse $t_{k}$ of $\mu_{k}$ is defined from $\left[-\mu_{0} / 2, \mu_{0} / 2\right]$ to $L_{k}$.

\section{PROPERTIES OF THE MAPS $G_{n, m}^{t}$ FOR $t$ IN $L_{k}$}

Observe that with the notation introduced in the previous section we have $n_{t}=n_{k}$ and $m_{t}=m_{k}$ for all $t \in L_{k}$. Thus, for $t \in L_{k}$, the maps $G_{n, m}^{t}$ in (2.1) can be written as follows:

$$
G_{n, m}^{t}(z)=\beta_{t}^{n}\left(\Delta_{k}(t) \lambda_{t}^{m} \Pi(z)+1+\mu_{k}(t)\right), \quad t \in L_{k} .
$$

Recall that $\Delta_{k}(t)$ can be taken arbitrarily close to 1 (it is sufficient to take $s$ in (5.12) small enough). As a consequence of the results in Section 3 we have the following:

Proposition 6.1. Given $\varepsilon>0$ there is $k_{0}$ such that for every $k \geq k_{0}$ and $t \in L_{k}$ the maps $T_{1,0}^{\mu_{k}(t)}$ and $G_{1,0}^{t}$ and $T_{0,1}^{\mu_{k}(t)}$ and $G_{0,1}^{t}$ are $\varepsilon$-close in the $C^{1}$-topology. In particular, for $G_{0,1}^{t}$ the continuations $\widetilde{A}_{t}$ and $\widetilde{R}_{t}$ of the hyperbolic fixed points $\widetilde{A}_{\mu_{k}(t)}$ and $\widetilde{R}_{\mu_{k}(t)}$ of $T_{0,1}^{\mu_{k}(t)}$ are well defined.

Moreover, $G_{1,0}^{a_{k}}\left(\widetilde{A}_{a_{k}}\right)<\widetilde{R}_{a_{k}}$ and $G_{1,0}^{b_{k}}\left(\widetilde{A}_{b_{k}}\right)>\widetilde{R}_{b_{k}}$. Thus there is $c_{k}$ such that $G_{1,0}^{c_{k}}\left(\widetilde{A}_{c_{k}}\right)=\widetilde{R}_{a_{k}}$, that is, the family $\left(G_{n, m}^{t}\right)_{t \in L_{k}}$ of maps has a cycle at $c_{k}$ associated to $\widetilde{A}_{c_{k}}$ and $\widetilde{R}_{c_{k}}$.

Finally, $T_{n, m}^{\mu_{k}(t)}$ and $G_{n, m}^{t}$ are $\varepsilon$-close in the $C^{0}$-topology for all $n$ and $m$.

Proposition 6.1 gives the following:

REMARK 6.2. The family $\mathcal{G}^{t}=\left(G_{n, m}^{t}\right)_{t \in L_{k}}$ of maps satisfies conditions (E1) - (E11) and (E13).

In Lemma 6.4 we will obtain a cycle condition analogous to (E12) (now the cycle occurs at $c_{k}$ ) for the family $\mathcal{G}^{t}$. In fact, in Lemma 6.4 we will prove that such a cycle is unfolded with scaled positive velocity (recall Lemma 3.12 where this result is stated for the cycles of $T_{n, m}^{\mu}$ ). In particular, this implies that $c_{k}$ is unique if $\mu_{0}$ is small.

As in Section 3 we define the maximal interval of definition $K_{n, m}^{t}$ of $G_{n, m}^{t}$, the admissible sequences $\left(n_{j}, m_{j}\right)$ for a point $x$, the orbit $x_{n_{j}, m_{j}}^{t}$, the position $a(x)$ of $x \in I$ and its itinerary $i_{n_{j}, m_{j}}^{t}(x)$. 
Remark 6.2 allows us to translate Lemma 3.4 to the families $G_{n, m}^{t}$ for $t \in L_{k}$.

Lemma 6.3. Consider $t \in L_{k}$ for large $k$, and $x \in I_{1} \cup I_{2}$ such that $G_{n, m}^{t}(x)$ is defined (i.e., $\left.x \in K_{n, m}^{t}\right)$. Then:

(1) If $m=1$ then $n=0$ and $a\left(G_{0,1}^{t}(x)\right)=1$ if $x \in I_{1}$, while it is 1 or 2 if $x \in I_{2}$.

(2) If $m=0$ then $n=1$ and $a\left(G_{1,0}^{t}(x)\right)=1$ if $x \in I_{1}$, while it is 1 or 2 if $x \in I_{2}$.

(3) If $m \geq 2$ then $n=0$ and $a\left(G_{0, m}^{t}(x)\right)=1$.

By Lemma 6.3 the orbits of points $x_{n_{j}, m_{j}}^{t}$ such that $i_{n_{j}, m_{j}}^{t}(x)=2$ for all $j$ only involve iterations under $G_{1,0}^{t}$ and $G_{0,1}^{t}$.

6.1. Cycles associated to $\widetilde{A}_{t}$ and $\widetilde{R}_{t}$. In the next lemma we prove that there is $c_{k} \in L_{k}$ with a cycle associated to $\widetilde{A}_{c_{k}}$ and $\widetilde{R}_{c_{k}}$. In order to prove the existence of such a cycle we need to use condition (E13). In fact, in the proof of Lemma 6.4 we will see that $\beta$ is the scaled velocity of the relative motion of $\widetilde{R}_{t}$ and $G_{1,0}^{t}\left(\widetilde{A}_{t}\right)$. Observe that the coexistence of the hyperbolic points $\widetilde{A}_{t}$ and $\widetilde{R}_{t}$ occurs only in a very small (scaled) interval of the parameter space. Thus to detect the unfolding of the cycle we need a large $\beta$. Our estimates can be improved in order to get a smaller value of $\beta$, but this is not relevant in view of the statement of the theorem.

As in Lemma 3.12 we consider the maps

$$
U_{k}: L_{k} \rightarrow \mathbb{R}, \quad U_{k}(t)=G_{1,0}^{t}\left(\widetilde{A}_{t}\right)-\widetilde{R}_{t}
$$

Clearly, $U_{k}(c)=0$ or $G_{1,0}^{c}\left(\widetilde{A}_{c}\right)=\widetilde{R}_{c}$ gives a cycle at $c$. The scaled velocity of the unfolding of the cycle is $c d U_{k}(c) / d t$.

LEMMa 6.4. For every $k$ sufficiently large there exists $c_{k} \in L_{k}$ such that $G_{1,0}^{c_{k}}\left(\widetilde{A}_{c_{k}}\right)=\widetilde{R}_{c_{k}}$ and $\mathcal{G}^{t}$ unfolds such a cycle with positive scaled velocity of order of $\beta$.

Proof. To get the cycle it is enough to see that there is $t \in L_{k}$ such that

$$
\beta(t)^{-1}\left(\widetilde{R}_{t}\right)=G_{0,0}^{t}\left(\widetilde{A}_{t}\right) .
$$

The map $U_{k}$ defined in $L_{k}$ is of the form

$$
U_{k}(t)=\beta(t) G_{0,0}^{t}\left(\widetilde{A}_{t}\right)-\widetilde{R}_{t}=G_{1,0}^{t}\left(\widetilde{A}_{t}\right)-\widetilde{R}_{t} .
$$

To prove (6.1), i.e., $U_{k}(t)=0$ for some $t \in L_{k}$, it is enough to see that

$$
U_{k}\left(a_{k}\right) \leq-\mu_{0} / 4 \text { and } U_{k}\left(b_{k}\right) \geq \mu_{0} / 4 \text {. }
$$


By the definition of $G_{n, m}^{t}$ for $t \in L_{k}$ (see (2.1) and Lemma 5.7), one has

$$
\begin{aligned}
U_{k}\left(a_{k}\right)= & \beta\left(a_{k}\right)\left(\Delta_{k}\left(a_{k}\right) \Pi\left(\widetilde{A}_{a_{k}}\right)+1-\mu_{0} / 2\right)-\widetilde{R}_{a_{k}} \\
= & \Delta_{k}\left(a_{k}\right)\left(\beta\left(a_{k}\right) \Pi\left(\widetilde{A}_{a_{k}}\right)+\beta\left(a_{k}\right)-\widetilde{R}_{a_{k}}\right) \\
& +\widetilde{R}_{a_{k}}\left(\Delta_{k}\left(a_{k}\right)-1\right)-\beta\left(a_{k}\right) \mu_{0} / 2 .
\end{aligned}
$$

Observe that

- $\frac{1-s}{1+s} \leq \Delta_{k}(t) \leq \frac{1+s}{1-s}$ for all $t \in L_{k}($ see $(5.12))$,

- the map $F(k)=\beta\left(a_{k}\right) \Pi\left(\widetilde{A}_{a_{k}}\right)+\beta\left(a_{k}\right)-\widetilde{R}_{a_{k}}$ tends to zero as $k$ goes to infinity (this follows from the cycle condition (E12)),

- $\widetilde{R}_{a_{k}}$ is close to $\widetilde{R}$ and $0<\beta^{-1}<\widetilde{R}<2$,

- $\beta\left(a_{k}\right)>\beta / 2$.

Therefore,

$$
\begin{aligned}
\widetilde{R}_{a_{k}}\left(\Delta_{k}\left(a_{k}\right)-1\right)-\beta\left(a_{k}\right) \frac{\mu_{0}}{2} & \leq 4\left(\frac{1+s}{1-s}-1\right)-\beta \frac{\mu_{0}}{4} \\
& =\frac{\mu_{0}}{4}\left(16 \frac{2 s}{1-s} \cdot \frac{1}{\mu_{0}}-\beta\right) .
\end{aligned}
$$

Using the definition of $s=s(\varepsilon)$ in (5.11) and of $\mu_{1}$ in (5.15) it is immediate to verify that

$$
\lim _{\varepsilon \rightarrow 0} \frac{s}{1-s} \cdot \frac{1}{\mu_{1}}=4
$$

Thus, by (5.17), $\lim _{\varepsilon \rightarrow 0} \mu_{1} /\left(-\mu_{2}\right)=1$ and since $\mu_{0}=\min \left\{\mu_{1},-\mu_{2}\right\}$, we have

$$
\lim _{\varepsilon \rightarrow 0} \frac{s}{1-s} \cdot \frac{1}{\mu_{0}}=4
$$

Therefore, for sufficiently small $\varepsilon$, one has

$$
\frac{s}{1-s} \cdot \frac{1}{\mu_{0}} \leq \frac{65}{16} \text {. }
$$

Now, from (6.3) and (6.4), we get

$$
U_{k}\left(a_{k}\right) \leq \Delta_{k}\left(a_{k}\right) F(k)+\frac{\mu_{0}}{4}(130-\beta) \leq-\frac{\mu_{0}}{4}
$$

if $k$ is sufficiently large and $\beta>131$ (condition (E13)).

Exactly in the same way we get

$$
U_{k}\left(b_{k}\right) \geq \Delta_{k}\left(b_{k}\right) \widetilde{F}(k)+\frac{\mu_{0}}{4}\left(\beta^{-1} \frac{-2 s}{1+s} \cdot \frac{1}{\mu_{0}}+\beta\right)
$$

where

$$
\widetilde{F}(k)=\beta\left(b_{k}\right) \Pi\left(\widetilde{A}_{b_{k}}\right)+\beta\left(b_{k}\right)-\widetilde{R}_{b_{k}} .
$$

As above, by (E12), $\widetilde{F}_{k}$ tends to zero as $k$ goes to infinity. 
We claim that if $k$ is large enough then

$$
U_{k}\left(b_{k}\right)>\Delta_{k}\left(b_{k}\right) \widetilde{F}(k)+\mu_{0} / 4 .
$$

To prove the claim it is enough to see that

$$
\beta^{-1} \frac{-2 s}{1+s} \cdot \frac{1}{\mu_{0}}+\beta>1 .
$$

From (6.5) taking $\varepsilon$ small we have

$$
\frac{s}{1-s} \cdot \frac{1}{\mu_{0}} \leq \frac{9}{2}
$$

Thus, since $(1+s) /(1-s)>1$, to prove the claim it is enough to see that

$$
\beta-9 \beta^{-1}>1 \text {, }
$$

which holds when $\beta>4$.

Finally, since $\Delta_{k}\left(b_{k}\right)$ is bounded and $\widetilde{F}(k) \rightarrow 0$ as $k \rightarrow \infty$, we have $U_{k}\left(b_{k}\right) \geq \mu_{0} / 4$ for sufficiently large $k$.

Now, by the mean value theorem, there is $c_{k}$ such that $U_{k}\left(c_{k}\right)=0$. This ends the proof of the first part of the lemma (existence of a cycle).

We now see that the arc unfolds such a cycle with scaled positive velocity of order of $\beta$. Recall that if $t \in L_{k}$ then

$$
G_{0,1}^{t}(\cdot)=\beta(t) \Delta_{k}(t) \Pi(\cdot)+\beta(t)\left(1+\mu_{k}(t)\right) .
$$

Recalling that

$$
U_{k}(t)=\beta(t) G_{0,0}^{t}\left(\widetilde{A}_{t}\right)-\widetilde{R}_{t}=G_{1,0}^{t}\left(\widetilde{A}_{t}\right)-\widetilde{R}_{t} .
$$

one has

$$
\begin{aligned}
\frac{d U_{k}}{d t}(t)= & \frac{d \beta}{d t}(t) \Delta_{k}(t) \Pi\left(\widetilde{A}_{t}\right)+\beta(t) \frac{d \Delta_{k}}{d t}(t) \Pi\left(\widetilde{A}_{t}\right) \\
& +\beta(t) \Delta_{k}(t) D \Pi\left(\widetilde{A}_{t}\right) \frac{d \widetilde{A}}{d t}(t)+\frac{d \beta}{d t}(t)\left(1+\mu_{k}(t)\right) \\
& +\beta(t) \frac{d \mu_{k}}{d t}(t)-\frac{d \widetilde{R}}{d t}(t) .
\end{aligned}
$$

To estimate this sum observe that

- $d \Delta_{k}(t) / d t$ is of order of $n_{k}-m_{k}$ and, by (5.1), this sum is of order of $-m_{k}$,

- the maps $\Delta_{k}, \beta$ and $\Pi$ are uniformly bounded,

- the derivative $d \mu_{k}(t) / d t$ is of order of $1 / t$ (see Lemma 5.6),

- the derivatives $D \Pi, d \beta / d t, d \widetilde{A} / d t$ and $d \widetilde{R} / d t$ are uniformly bounded (recall the proof of Lemma 3.12).

In view of these considerations we have

$$
\frac{d U_{k}}{d t} \simeq \text { const }_{0}-\text { const }_{1} m_{k}+\text { const }_{2} \frac{\beta}{t_{k}} .
$$


Since $t$ is in the scaled interval $L_{k}^{*}$, and hence it is of order of $t_{k}=\lambda\left(t_{k}\right)^{-m_{k}}$, we have

$$
t \frac{d U_{k}}{d t} \simeq \lambda\left(t_{k}\right)^{-m_{k}}\left(\text { const }-m_{k}\right)+\beta\left(t_{k}\right) \simeq \beta\left(t_{k}\right),
$$

where the last relation follows by observing that $\lambda\left(t_{k}\right)^{-m_{k}} m_{k} \rightarrow 0$ as $k \rightarrow \infty$ and $t \simeq t_{k}$ if $t \in L_{k}$.

Let us make a comment about the proof of Lemma 6.4 which follows immediately from (6.6).

REMARK 6.5. There is a unique $c_{k} \in L_{k}$ such that $U_{k}\left(c_{k}\right)=0$. Moreover,

$$
\begin{array}{ll}
G_{1,0}^{t}\left(\widetilde{A}_{t}\right)>\widetilde{R}_{t} & \text { for all } \left.t \in] c_{k}, b_{k}\right], \\
G_{1,0}^{t}\left(\widetilde{A}_{t}\right)<\widetilde{R}_{t} & \text { for all } t \in\left[a_{k}, c_{k}[.\right.
\end{array}
$$

Recalling that $U_{k}\left(a_{k}\right) \leq-\mu_{0} / 4$ and $U_{k}\left(b_{k}\right) \geq \mu_{0} / 4$ (see (6.2)), and $U_{k}\left(c_{k}\right)=0$, we get

$$
\begin{aligned}
& \mu_{0} / 4 \leq\left|U_{k}\left(b_{k}\right)-U_{k}\left(c_{k}\right)\right|=\left|U_{k}^{\prime}(\psi)\right| \cdot\left|b_{k}-c_{k}\right|, \\
& \mu_{0} / 4 \leq\left|U_{k}\left(a_{k}\right)-U_{k}\left(c_{k}\right)\right|=\left|U_{k}^{\prime}(\xi)\right| \cdot\left|a_{k}-c_{k}\right| .
\end{aligned}
$$

Now (6.6) and the definition of $L_{k}$ imply that

$$
\frac{\left|b_{k}-c_{k}\right|}{t_{k}} \geq C \frac{\mu_{0}}{4 \beta} \quad \text { and } \quad \frac{\left|a_{k}-c_{k}\right|}{t_{k}} \geq C \frac{\mu_{0}}{4 \beta},
$$

for some $C>0$ independent of $k$.

The next lemma is a direct consequence of (6.7) and (5.2).

Lemma 6.6. The sequences $a_{k}, b_{k}$ and $c_{k}$ satisfy

$$
\liminf _{t \rightarrow 0^{+}} \frac{\left|\left(\bigcup_{k}\left[c_{k}, b_{k}\right]\right) \cap[0, t]\right|}{t}>0 \quad \text { and } \quad \liminf _{t \rightarrow 0^{+}} \frac{\left|\left(\bigcup_{k}\left[a_{k}, c_{k}\right]\right) \cap[0, t]\right|}{t}>0 \text {. }
$$

Observe that the intervals $\left.] c_{k}, b_{k}\right]$ and $\left[a_{k}, c_{k}\right.$ [ will be the parameter intervals in (5) in the statement of the theorem.

As in Section 3.1 denote by $\Omega_{i}^{t}$ the set $\Omega\left(\mathcal{G}^{t}\right) \cap I_{i}, i=\{1, \ldots, 4\}$. Using Lemmas 6.3 and 6.4 and formula (6.5) one gets the following reformulation of Proposition 3.7 and Corollaries 3.11 and 4.12.

Proposition 6.7. Let $t \in L_{k}$ with $k$ sufficiently large. Then:

(a) If $z \in \Omega_{1}^{t}$ then $z \in \omega^{t}(x)$ for all $x \in I_{1}$.

(b) If $z \in \Omega_{2}^{t}$ then $z \in\left[\widetilde{A}_{t}, \widetilde{R}_{t}\right]$. Moreover, if $t \geq c_{k}$ then $z=\widetilde{A}_{t}$ or $\widetilde{R}_{t}$.

(c) If $z \in \Omega_{3}^{t}$ then there is a $\mathcal{G}^{t}$-orbit $z_{n_{j}, m_{j}}^{t}$ of $z$ such that $i_{n_{j}, m_{j}}^{t}(z)=3$ for all $j \geq 0$.

(d) $\Omega_{4}^{t}=\emptyset$.

(e) Let $x \in I_{1}$ and $x_{n, m}^{t} \in I_{1}$. Then $n=0$ or 1 and

$$
0<\left(G_{n, m}^{t}\right)^{\prime}(x)<\beta \varrho^{\mathrm{s}}=\bar{\varrho}<1 .
$$


(f) Let $x \in I_{3}$ and $x_{n, m}^{t} \in I_{3}$. Then $m=0$ or 1 and

$$
\left(G_{n, m}^{t}\right)^{\prime}(x)>\lambda \varrho^{\mathrm{u}}=\varrho>1 .
$$

(g) If $\left.t \in] c_{k}, b_{k}\right]$ then $\Omega\left(\mathcal{G}^{t}\right)=\Omega_{1}^{t} \cup \Omega_{3}^{t} \cup\left\{\widetilde{A}_{t}, \widetilde{R}_{t}\right\}$.

(h) For every $t \in\left[a_{k}, c_{k}\left[, \Omega_{2}^{t}=\left[\widetilde{A}_{t}, \widetilde{R}_{t}\right]\right.\right.$ and $\Omega\left(\mathcal{G}^{t}\right)=\Omega_{1}^{t} \cup \Omega_{3}^{t} \cup\left[\widetilde{A}_{t}, \widetilde{R}_{t}\right]$.

6.2. How things work in higher dimensions. Recall that the maps $G_{n, m}^{t}$ give the central coordinate of returns of points to the reference neighbourhood $\mathcal{V}_{1}$. Bearing this in mind we will obtain the following:

1. For $t \in L_{k}$ the points $\widetilde{A}_{t}$ and $\widetilde{R}_{t}$ are the central coordinates of two hyperbolic periodic points $A_{t}$ and $R_{t}$ of indices $r+1$ and $r$ and period $m_{t}+N_{2}+n_{t}+1+N_{1}$ (see Lemma 8.1).

2. For each $k$ the parameter $c_{k}$ corresponds to a heterodimensional cycle associated to $A_{t}$ and $R_{t}$ (see Lemma 8.2).

3. For every $\left.t \in] c_{k}, b_{k}\right]$ the homoclinic classes of $P_{t}$ and $Q_{t}, \overline{H\left(P_{t}\right)}$ and $\overline{H\left(Q_{t}\right)}$, are hyperbolic and the resulting nonwandering set after the bifurcation is $\Omega\left(f_{t}\right)^{\prime}=\overline{H\left(P_{t}\right)} \cup \overline{H\left(Q_{t}\right)} \cup \mathcal{O}\left(A_{t}\right) \cup \mathcal{O}\left(R_{t}\right)$ (see Propositions 7.11 and 7.14 and Lemma 8.3).

4. Observe that, for $t \in\left[c_{k}, b_{k}\right]$, the point $\beta(t)^{n_{t}} t$ corresponds to the central coordinate of the homoclinic point of $P_{t}$ which is the continuation of the heteroclinic point $X_{0}$. Thus every $G_{n, m}^{t}\left(\beta(t)^{n_{t}}\right)$ (if well defined) corresponds to the central coordinate of some homoclinic point of $P_{t}$.

5. If $X \in H\left(Q_{t}\right)$ and its central coordinate $X^{\mathrm{c}}$ belongs to $I$ then $X^{\mathrm{c}}$ is accumulated by the left extremes of the intervals $K_{n, m}^{t}$.

6 . For every $t \in\left[a_{k}, c_{k}\right.$ [ one has $G_{1,0}^{t}\left(\widetilde{A}_{t}\right) \in\left(\widetilde{A}_{t}, \widetilde{R}_{t}\right)$ and this point is the central coordinate of the homoclinic point of $\widetilde{A}_{t}$ associated to the unfolding of the cycle at $c_{k}$.

7. For every $t \in\left[a_{k}, c_{k}\right.$ [ the resulting nonwandering set is $\Omega\left(f_{t}\right)^{\prime}=$ $\overline{H\left(P_{t}\right)} \cup \overline{H\left(Q_{t}\right)} \cup \overline{H\left(\widetilde{A}_{t}\right)}$ (disjoint union; see Section 9).

\section{THE HOMOCLINIC CLASSES ASSOCIATED TO $P_{t}$ AND $Q_{t}$}

We first prove the theorem for the special family constructed in Section 1.3 and then we explain why it remains valid for a neighbourhood of this family (see Section 7.3).

7.1. Returns and associated sequences. In what follows we fix a large $k$ and a parameter $t \in L_{k}$. Given $Z=\left(z^{\mathrm{s}}, z^{\mathrm{c}}, z^{\mathrm{u}}\right) \in \mathcal{V}_{1}$, its central coordinate, denoted by $Z^{\mathrm{c}}$, is the point of coordinates $\left(0^{\mathrm{s}}, z^{\mathrm{c}}, 0^{\mathrm{u}}\right)$. Define

$$
\mathcal{I}_{i}= \begin{cases}\left\{Z \in \mathcal{V}_{1}: Z^{\mathrm{c}} \in I_{i}\right\} & \text { if } i=1,3 \\ \left\{Z \in \mathcal{V}_{1}: Z^{\mathrm{c}} \in \operatorname{int}\left(I_{i}\right)\right\} & \text { if } i=2,4\end{cases}
$$


For simplicity assume that $\mathcal{V}_{1}$ is the union of the four sets above. Observe that every $Z$ in $\Lambda_{t}=\Lambda_{t}(\mathcal{V})=\bigcap_{n \in \mathbb{Z}} f_{t}^{n}(\mathcal{V})$ (see (1.3)) different from $P_{t}$ and $Q_{t}$ has a return in $\mathcal{I}_{1} \cup \ldots \cup \mathcal{I}_{4}$. Given $Z \in \mathcal{V}_{1}$ we define the position $a_{0}(Z)$ of $Z$ by

$$
a_{0}(Z)=i \quad \text { if } Z \in \mathcal{I}_{i}, i \in\{1,2,3,4\} .
$$

Suppose now that $Z \in \mathcal{V}_{1} \cap \Lambda_{t}$. If $Z$ returns to $\mathcal{V}_{1}$ under positive iterations then we define the forward return time of $Z$ to $\mathcal{V}_{1}$, denoted by $r_{1}=r_{1}(Z)$, as the first positive integer such that $f_{t}^{r_{1}}(Z) \in \mathcal{V}_{1}$. Set

$$
Z_{j}=f_{t}^{j}(Z) \text { and } Z_{0}=Z \text {. }
$$

We define $a_{1}(Z) \in\{1,2,3,4\}$ according to the position of $Z_{r_{1}}$ as before, i.e.,

$$
a_{1}(Z)=a_{0}\left(Z_{r_{1}}\right) \text {. }
$$

If the forward orbit of $Z_{r_{1}}$ returns to $\mathcal{V}_{1}$ we let

$$
r_{2}=r_{2}(Z)=r_{1}\left(Z_{r_{1}}\right) \text { and } a_{2}(Z)=a_{1}\left(Z_{r_{1}}\right)=a_{0}\left(Z_{r_{2}}\right) .
$$

In this way, if the orbit of $Z$ returns $i+1$ times to $\mathcal{V}_{1}$, for $j \leq i$ we define inductively the forward returns $r_{j}=r_{j}(Z)$ and positions $a_{j}(Z)$ of $Z$ as follows:

$$
\begin{aligned}
& r_{j+1}(Z)=r_{1}\left(Z_{r_{j}}\right)=r_{j}\left(Z_{r_{1}}\right), \\
& a_{j+1}(Z)=a_{0}\left(Z_{r_{j+1}}\right)=a_{1}\left(Z_{r_{j}}\right)=a_{j}\left(Z_{r_{1}}\right) .
\end{aligned}
$$

It is clear that we can do the same for the backward orbit of $Z$, defining, if possible, $r_{i}=r_{i}(Z)$ and $a_{i}(Z)$ for negative $i$. For completeness we let $r_{0}(Z)=0$.

Given $Z \in \mathcal{V}_{1} \cap \Lambda_{t}$ let $I(Z)$ be the maximal set of integers such that if $i_{1}$ and $i_{2}, i_{1} \leq i_{2}$, belong to $I(Z)$ then $r_{i}(Z)$ is defined for all integers $i$ with $i_{1} \leq i \leq i_{2}$. Now to each point $Z$ of $\mathcal{V}_{1} \cap \Lambda_{t}$ we associate the sequences $\left(r_{j}(Z)\right)_{j \in I(Z)}$ of return times, $\left(a_{j}(Z)\right)_{j \in I(Z)}$ of positions and $\left(Z_{r_{j}}\right)_{j \in I(Z)}$ of iterates.

Due to the geometry of the cycle it is easy to get the following lemma whose proof we omit.

Lemma 7.1. Consider small $t>0$ and $Z \in \Lambda_{t} \cap \mathcal{V}_{1}$. Then:

- $I(Z)=\{0\}$ if and only if $Z \in \gamma$.

- $I(Z)$ is upper bounded if and only if $Z \in W^{\mathrm{s}}\left(P_{t}\right) \cup W^{\mathrm{s}}\left(Q_{t}\right)$.

- $I(Z)$ is lower bounded if and only if $Z \in W^{\mathrm{u}}\left(P_{t}\right) \cup W^{\mathrm{u}}\left(Q_{t}\right)$.

Let us also state the following remark, which is a consequence of the existence of the filtration (condition (C7)) and the geometry of the cycle.

REMARK 7.2. For every $t>0$ small enough we have:

- If $Z=\left(z^{\mathrm{s}}, z^{\mathrm{c}}, z^{\mathrm{u}}\right)$ belongs to $\mathcal{U}_{Q} \cap \Omega\left(f_{t}\right)^{\prime}$ then $z^{\mathrm{c}} \geq 0$. Similarly, if $Z \in\left(\mathcal{U}_{P} \cap \Omega\left(f_{t}\right)^{\prime}\right)$ then $z^{\mathrm{c}} \leq 0$. 
- Suppose that $Z \in \mathcal{V}$ and that $f_{t}^{i}(Z)$ is a forward (resp. backward) iterate of $Z$ which does not belong to $\mathcal{V}$. Then there is $j_{0}=j_{0}(Z)$ such that $f_{t}^{j}(Z) \notin \mathcal{V}$ for all $j>j_{0}$ (resp. $j<j_{0}$ ).

7.1.1. Central dynamics. In this section we analyze the dynamics of the returns to $\mathcal{V}_{1}$. The central coordinate of such returns is given by the maps $G_{n, m}^{t}$ defined in (2.1) (see also (7.1) above).

Consider a point $Z \in \mathcal{V}_{1}$ such that $1 \in I(Z)$. Let $r_{1}=r_{1}(Z)>0$ and recall that we split the segment $\left\{0,1, \ldots, r_{1}\right\}$ of the orbit of $Z$ as follows:

$$
r_{1}=r_{1}(Z)=q_{1}(Z)+N_{2}+p_{1}(Z)+N_{1},
$$

where $N_{1}$ and $N_{2}$ are the transition times from $\mathcal{U}_{Q}$ to $\mathcal{U}_{P}$ and from $\mathcal{U}_{P}$ to $\mathcal{U}_{Q}$, respectively, and $p_{1}=p_{1}(Z)$ and $q_{1}=q_{1}(Z)$ are the numbers of consecutive iterates of this orbit segment in $\mathcal{U}_{P}$ and $\mathcal{U}_{Q}$, respectively. We claim that if $t \in L_{k}$ then

$$
p_{1} \geq-m_{k} \quad \text { and } \quad q_{1} \geq n_{k}
$$

(see (5.1) for the definitions of $n_{k}$ and $m_{k}$ ).

To see (for instance) that $p_{1} \geq-m_{k}$ observe that, by definition of $p_{1}$, $Z_{i} \in \mathcal{U}_{P}$ for all $i \in\left\{N_{1}, \ldots, p_{1}+N_{1}\right\}$ and $Z_{p_{1}+N_{1}} \in f_{t}^{-N_{2}}\left(\mathcal{V}_{2}\right)$. By the construction of the arc and (F2),

$$
z_{N_{2}+p_{1}+N_{1}}^{\mathrm{c}}=z_{p_{1}+N_{1}}^{\mathrm{c}}+t=\lambda(t)^{p_{1}} z_{N_{1}}^{\mathrm{c}}+t .
$$

Remark 7.2 and the fact that $Z_{N_{2}+p_{1}+N_{1}} \notin W_{\text {loc }}^{\mathrm{s}}\left(Q_{t}\right)$ (otherwise the orbit of $Z$ does not return to $\left.\mathcal{V}_{1}\right)$ imply that $z_{p_{1}+N_{1}}^{\mathrm{c}}+t>0$. Thus $z_{p_{1}+N_{1}}^{\mathrm{c}}>-t$. Also recall that $z_{N_{1}}^{\mathrm{c}}$ belongs to $J=[-b,-\lambda b]$. Thus

$$
\lambda(t)^{p_{1}}(-\lambda b) \geq \lambda(t)^{p_{1}} z_{N_{1}}^{\mathrm{c}}>-t \geq \frac{-1}{1-s} \lambda(t)^{-m_{k}},
$$

where the last inequality follows from (5.7). As $b$ and $s$ are close to 1 and 0 , respectively, we conclude that $p_{1}+1>-m_{k}$, that is, $p_{1} \geq-m_{k}$. Analogously one proves that $q_{1} \geq n_{k}$.

From the previous arguments it follows that there are $n(Z), m(Z) \in \mathbb{N}_{0}$ such that

$r_{1}=q_{1}+N_{2}+p_{1}+N_{1}$, where $q_{1}=n_{k}+n(Z)$ and $p_{1}=\left(-m_{k}\right)+m(Z)$, where

- $Z_{i} \in \mathcal{U}_{P}$ for all $i \in\left\{N_{1}, N_{1}+1, \ldots, N_{1}+p_{1}\right\}$,

- $Z_{p_{1}+N_{1}} \in f_{t}^{-N_{2}}\left(\mathcal{V}_{2}\right)$,

- $Z_{i} \in \mathcal{U}_{Q}$ for all $i \in\left\{N_{1}+p_{1}+N_{2}, \ldots, N_{1}+p_{1}+N_{2}+q_{1}\right\}$.

In this case we say that $r_{1}$ is a return of type $(n(Z), m(Z))$.

By definition, if $Z_{i}=\left(z_{i}^{\mathrm{s}}, z_{i}^{\mathrm{c}}, z_{i}^{\mathrm{u}}\right)$ belongs to $\mathcal{U}_{P} \cup \mathcal{U}_{Q}$ then $Z_{i}^{\mathrm{c}}=\left(0^{\mathrm{s}}, z_{i}^{\mathrm{c}}, 0^{\mathrm{u}}\right)$. Observe that by the $f_{t}$-invariance of the foliations (condition $(\mathrm{F} 1)$ ), the definitions of $N_{1}$ and $\Pi$, and our construction, we have 
- $Z_{N_{1}}^{\mathrm{c}}=\Pi\left(Z^{\mathrm{c}}\right)$

- $Z_{p_{1}+N_{1}}^{\mathrm{c}}=\lambda(t)^{p_{1}} Z_{N_{1}}^{\mathrm{c}}$,

- $Z_{N_{2}+p_{1}+N_{1}}^{\mathrm{c}}=H^{t}\left(Z_{p_{1}+N_{1}}^{\mathrm{c}}\right)=\lambda(t)^{p_{1}} Z_{N_{1}}^{\mathrm{c}}+t=\lambda(t)^{p_{1}} \Pi\left(Z^{\mathrm{c}}\right)+t$.

Therefore, using the definitions of $p_{1}$ and $q_{1}$, one has

$$
Z_{r_{1}}^{\mathrm{c}}=\beta(t)^{n(Z)+n_{k}}\left(\lambda(t)^{m(Z)-m_{k}} \Pi\left(Z^{\mathrm{c}}\right)+t\right) .
$$

Using (5.12) and (5.14) and since $t \in L_{k}$ we get

$$
G_{n(Z), m(Z)}^{t}\left(Z^{\mathrm{c}}\right)=Z_{r_{1}}^{\mathrm{c}}=\beta(t)^{n(Z)}\left(\lambda(t)^{m(Z)} \Delta_{k}(t) \Pi\left(Z^{\mathrm{c}}\right)+1+\mu_{k}(t)\right),
$$

where $\mu_{k}(t) \in\left[-\mu_{0} / 2, \mu_{0} / 2\right]$ (recall Lemma 5.7).

Thus the family of the functions $G_{n, m}^{t}$ defines the central dynamics for points having a forward return of type $(n, m)$.

Define $\mathcal{V}_{n, m}^{t}$ as the set of points in $\mathcal{V}_{1}$ having a return of type $(n, m)$. Observe that this set is an $n$-dimensional box such that $\left\{Z^{\mathrm{c}} \in \mathcal{V}_{n, m}^{t}\right\}=$ $K_{n, m}^{t}$, where $K_{n, m}^{t}$ is defined as in Section 3 .

7.1.2. Itineraries. The next lemma characterizes the sequences of possible positions for points $Z \in \Lambda_{t} \cap \mathcal{V}_{1}$ for $t \in L_{k}$ and is a consequence of the comments above and of Lemmas 3.4 and 3.6 for the $G_{n, m}^{t}$ maps.

Lemma 7.3. Given $t \in L_{k}$ consider $Z \in \Lambda_{t} \cap \mathcal{V}_{1}$ and $j \in I(Z)$. Then:

(1) If $a_{j}(Z)=1$ and $j+1 \in I(Z)$, then $a_{j+1}(Z)=1$ and $Z_{r_{j+1}} \in \operatorname{int}\left(\mathcal{I}_{1}\right)$.

(2) If $a_{j}(Z)=2$ and $j+1 \in I(Z)$, then $a_{j+1}(Z)=1$ or 2 .

(3) If $a_{j}(Z)=2$ and $j-1 \in I(Z)$, then $a_{j-1}(Z)=2$ or 3 .

(4) If $a_{j}(Z)=3$ and $j-1 \in I(Z)$, then $a_{j-1}(Z)=3$ and $Z_{r_{j-1}} \in \operatorname{int}\left(\mathcal{I}_{3}\right)$.

(5) If $a_{j}(Z)=4$ and $j+1 \in I(Z)$, then $a_{j+1}(Z)=1$ and $Z_{r_{j+1}} \in \operatorname{int}\left(\mathcal{I}_{1}\right)$.

(6) If $a_{j}(Z)=4$ and $j-1 \in I(Z)$, then $a_{j-1}(Z)=3$ and $Z_{r_{j-1}} \in \operatorname{int}\left(\mathcal{I}_{3}\right)$.

Observe that if $a_{j}(Z)=1$ and $j-1 \in I(Z)$, then $a_{j-1}(Z)$ (a priori) can take any value in $\{1,2,3,4\}$. The same holds when $a_{j}(Z)=3$ and $j+1 \in I(Z)$, i.e., $a_{j+1}(Z)=1,2,3$ or 4 .

REMARK 7.4. The previous lemma also holds for any $Z \in \mathcal{V}_{1}$ having a forward (resp. backward) return $r_{1}$ to $\mathcal{V}_{1}$ such that $Z, Z_{1}, \ldots, Z_{r_{1}}$ (resp. $\left.Z, Z_{-1}, \ldots Z_{r_{1}}\right)$ belong to $\mathcal{V}$.

Corollary 7.5. Consider $t \in L_{k}$ with $k$ large enough, and let $Z \in$ $\Omega\left(f_{t}\right) \cap \mathcal{V}_{1}$ be such that $I(Z)=\mathbb{Z}$. Then there are three possibilities:

(1) $a_{j}(Z)=1$ for every $j \in I(Z)$.

(2) $a_{j}(Z)=2$ for every $j \in I(Z)$.

(3) $a_{j}(Z)=3$ for every $j \in I(Z)$. 
Proof. First observe that if $Z \in \mathcal{I}_{i}$ and $f_{t}^{m}(Z) \in \operatorname{int}\left(\mathcal{I}_{j}\right)$ then there is a neighbourhood $V$ of $Z$ with the same property, i.e., $f_{t}^{m}(V) \subset \operatorname{int}\left(\mathcal{I}_{j}\right)$. The corollary now follows from Lemma 7.3 and Remark 7.4.

The next three lemmas are similar to Steps B, C and D of $\left[\mathrm{DR}_{2}\right.$, Section 4.2], therefore we just sketch their proofs. Observe that if $Z \in \Lambda_{t} \backslash\left\{P_{t}, Q_{t}\right\}$ then there exists $k=k(Z) \in \mathbb{Z}$ such that $f_{t}^{k}(Z) \in \mathcal{V}_{1}$. This means that by replacing $Z$ by some iterate we can (and do) assume that $Z \in \mathcal{V}_{1}$.

Lemma 7.6. Let $t \in L_{k}$ with $k$ sufficiently large.

(1) If $Z \in W^{\mathrm{u}}\left(P_{t}\right) \cap\left(\Omega\left(f_{t}\right) \cap \mathcal{V}_{1}\right)$ and $Z \neq P_{t}$, then $a_{j}(Z)=1$ for every $j \in I(Z)$.

(2) If $Z \in W^{\mathrm{s}}\left(Q_{t}\right) \cap\left(\Omega\left(f_{t}\right) \cap \mathcal{V}_{1}\right)$ and $Z \neq Q_{t}$, then $a_{j}(Z)=3$ for every $j \in I(Z)$.

Proof. Let us prove (1); the proof of (2) runs analogously by considering the backward orbit of $Z$, so we omit it.

Take any $Z$ as in the first item of the lemma. Then there is $l \in \mathbb{Z}$ such that $f_{t}^{l}(Z) \in W_{\text {loc }}^{\mathrm{u}}\left(P_{t}\right) \cap \mathcal{V}_{2}$. Thus $f_{t}^{N_{2}+l}(Z) \in f_{t}^{N_{2}}\left(W_{\text {loc }}^{\mathrm{u}}\left(P_{t}\right)\right) \cap \mathcal{U}_{Q}$. Recall now that $X_{t}=\left(x_{0}^{\mathrm{s}}, t, 0^{\mathrm{u}}\right)$ is the homoclinic point of $P_{t}$ defined as the the continuation of $X_{0}$ (see Section 1.3). Thus by (F1),

$$
\left(f_{t}^{N_{2}+l}(Z)\right)^{\mathrm{c}}=\left(0^{\mathrm{s}}, t, 0^{\mathrm{u}}\right)=X_{t}^{\mathrm{c}} .
$$

Write $W=f_{t}^{n_{k}+N_{2}+l}(Z)$. Then, by the definition of $n_{k}$ and Lemma 5.7, we have

$$
W^{\mathrm{c}}=f_{t}^{n_{k}}\left(X_{t}^{\mathrm{c}}\right)=\left(0^{\mathrm{s}}, \beta(t)^{n_{k}} t, 0^{\mathrm{u}}\right),
$$

where

$$
\beta(t)^{n_{k}} t \in\left[1-\mu_{0} / 2,1+\mu_{0} / 2\right] \subset \operatorname{int}\left(I_{1}\right) .
$$

Therefore $a_{0}(W)=1$ and $W \in \mathcal{I}_{1}$. By the arguments in the proof of Corollary $7.5, a_{j}(W)=1$ for all $j \in I(W)$ and $j \geq 0$.

By construction, $f_{t}^{-i}(W) \in \mathcal{U}_{P}$ for all $i \geq n_{k}+N_{2}+l$, thus $I(W)$ consists only of nonnegative numbers. This completes the proof of the result for the point $W$. The lemma follows by observing that there is $h$ such that $a_{j}(Z)=a_{j+h}(W)$ for all $j \in I(Z)$.

The previous lemma shows that every homoclinic point of $P_{t}$ (resp. of $\left.Q_{t}\right)$ in $\mathcal{V}_{1}$ is in the interior of $\mathcal{I}_{1}$ (resp. of $\mathcal{I}_{3}$ ). By continuity we have the following:

Corollary 7.7. Suppose that the point $Z$ belongs to the closure of $H\left(P_{t}\right)\left(\right.$ resp. $\left.H\left(Q_{t}\right)\right)$. Then $a_{j}(Z)=1\left(\right.$ resp. $\left.a_{j}(Z)=3\right)$ for all $j \in I(Z)$.

Lemma 7.8. Let $t \in L_{k}$ with $k$ sufficiently large. Then:

(1) $W^{\mathrm{u}}\left(P_{t}\right) \cap W^{\mathrm{s}}\left(Q_{t}\right) \cap\left(\Omega\left(f_{t}\right) \cap \mathcal{V}\right)=\emptyset$ (no-cycles condition).

(2) $W^{\mathrm{s}}\left(P_{t}\right) \cap W^{\mathrm{u}}\left(Q_{t}\right) \cap\left(\Omega\left(f_{t}\right) \cap \mathcal{V}\right)=\emptyset$. 
(3) The intersections between $W^{\mathrm{s}}\left(P_{t}\right)$ and $W^{\mathrm{u}}\left(P_{t}\right)$ and between $W^{\mathrm{s}}\left(Q_{t}\right)$ and $W^{\mathrm{u}}\left(Q_{t}\right)$ are transverse.

Proof. Item (3) follows easily from the geometry of the cycle and the existence of the filtration (conditions $(\mathrm{C} 1)-(\mathrm{C} 7)$ ); for details see $\left[\mathrm{DR}_{2}\right.$, Section $4.2]$.

To prove (1) we see that any $Z \in W^{\mathrm{u}}\left(P_{t}\right) \cap W^{\mathrm{s}}\left(Q_{t}\right)$ is wandering. We can assume that $Z \in \mathcal{V}_{1}$. On the one hand, if $Z$ is nonwandering, by Lemma 7.6(1), we have $a_{j}(Z)=1$ for all $j \in I(Z)$. On the other hand, by Lemma $7.6(2), a_{j}(Z)=3$ for all $j \in I(Z)$, which is a contradiction.

To prove (2) we first claim that if $Z \in W_{\text {loc }}^{\mathrm{s}}\left(P_{t}\right) \cap \mathcal{V}$ (resp. $Z \in W_{\text {loc }}^{\mathrm{u}}\left(Q_{t}\right)$ $\cap \mathcal{V}$ ) then there exists a neighbourhood $\mathcal{W}$ of $Z$ such that $a_{0}\left(W_{Y}\right)=1$ (resp. $a_{0}\left(W_{Y}\right)=3$ ) for every $Y \in \mathcal{W}$ whose forward orbit intersects $\mathcal{V}_{1}$; here $W_{Y}$ denotes the first forward (resp. backward) iterate of $Y$ in $\mathcal{V}_{1}$.

This claim can be seen as follows. Observe that (7.2) implies that $f_{t}^{n_{k}}\left(X_{t}\right)$ belongs to the interior of $\mathcal{I}_{1}$. Taking a small neighbourhood $\mathcal{W}$ of $Z$, by the $\lambda$-lemma, we see that $W_{Y}^{\mathrm{c}}$ is close to $\left(f_{t}^{n_{k}}\left(X_{t}\right)\right)^{\mathrm{c}}$, thus $W_{Y}$ is in the interior of $\mathcal{I}_{1}$. If $Z \in W_{\mathrm{loc}}^{\mathrm{u}}\left(Q_{t}\right) \cap \mathcal{V}$ the argument is similar by taking backward iterates.

If $Z \in W^{\mathrm{s}}\left(P_{t}\right) \cap W^{\mathrm{u}}\left(Q_{t}\right)$, then replacing it by some iterate, we can assume that $Z \in \mathcal{V}_{1}$.

First, if $Z \notin \mathcal{I}_{1}$, since $Z_{i} \in W_{\text {loc }}^{\mathrm{s}}\left(P_{t}\right)$ for all $i$ large enough, from the previous observation and Remark 7.4, there exists a neighbourhood $\mathcal{W}$ such that for every $Y \in \mathcal{W}$ one has $Y \notin \mathcal{I}_{1}$ and $a_{j}(Y)=1$ for all $j \in I(Y)$ with $j>0$. As in the proof of Corollary 7.5, this implies that $f_{t}^{n}(\mathcal{W}) \cap \mathcal{W}=\emptyset$ for every $n \in \mathbb{N}$. Thus $Z$ is wandering.

Second, if $Z \in \mathcal{I}_{1}$ then there exists a neighbourhood $\mathcal{W}$ such that $Y \notin \mathcal{I}_{3}$ for every $Y \in \mathcal{W}$. Since $Z_{-i} \in W_{\text {loc }}^{\mathrm{u}}\left(Q_{t}\right)$ for all $i$ large enough, by the arguments before we have $a_{j}(Y)=3$ for all $j<0$ in $I(Y)$. As above, it follows that $f_{t}^{-n}(\mathcal{W}) \cap \mathcal{W}=\emptyset$ for all $n \in \mathbb{N}$, hence $Z$ is wandering.

From the proof of the previous lemma we get the following fact:

Remark 7.9. Consider $t \in L_{k}$ with $k$ large. Then:

- If $Z \in W^{\mathrm{s}, \mathrm{u}}\left(P_{t}\right) \cap\left(\Omega\left(f_{t}\right) \cap \mathcal{V}_{1}\right)$ then $a_{j}(Z)=1$ for all $j \in I(Z)$.

- If $Z \in W^{\mathrm{s}, \mathrm{u}}\left(Q_{t}\right) \cap\left(\Omega\left(f_{t}\right) \cap \mathcal{V}_{1}\right)$ then $a_{j}(Z)=3$ for all $j \in I(Z)$.

Lemma 7.10. Let $t \in L_{k}$ with $k$ sufficiently large. If $Z \in \Omega\left(f_{t}\right) \cap \mathcal{V}_{1}$ then there are three possibilities:

(1) $a_{j}(Z)=1$ for every $j \in I(Z)$.

(2) $a_{j}(Z)=2$ for every $j \in I(Z)$.

(3) $a_{j}(Z)=3$ for every $j \in I(Z)$. 
Proof. There are four possibilities for the set $I(Z)$ : (i) $I(Z)=\mathbb{Z}$, (ii) $I(Z)$ is infinite and lower bounded, (iii) $I(Z)$ is infinite and upper bounded, and (iv) $I(Z)$ is finite.

In the first case the result follows from Corollary 7.5.

If $I(Z)$ is finite (case (iv)) then, by Lemma 7.1,

$$
Z \in\left(W^{\mathrm{s}}\left(P_{t}\right) \cup W^{\mathrm{s}}\left(Q_{t}\right)\right) \cap\left(W^{\mathrm{u}}\left(P_{t}\right) \cup W^{\mathrm{u}}\left(Q_{t}\right)\right) .
$$

As $Z$ is nonwandering, from Lemma 7.8, one gets

$$
Z \in W^{\mathrm{s}}\left(P_{t}\right) \pitchfork W^{\mathrm{u}}\left(P_{t}\right)=H\left(P_{t}\right) \quad \text { or } \quad Z \in W^{\mathrm{s}}\left(Q_{t}\right) \pitchfork W^{\mathrm{u}}\left(Q_{t}\right)=H\left(Q_{t}\right) .
$$

By Corollary 7.7, if $Z \in H\left(P_{t}\right)$ then $a_{j}(Z)=1$ for all $j \in I(Z)$, and if $Z \in H\left(Q_{t}\right)$ then $a_{j}(Z)=3$ for all $j \in I(Z)$.

Now consider case (ii) (case (iii) follows similarly). Observe first that, as in the proof of Corollary 7.5, one has $a_{j}(Z) \neq 4$ for all $j$. We now argue by contradiction: assume that the sequence of positions $a_{i}(Z)$ of $Z$ does not satisfy the conclusion of the lemma. Then (in particular) there exists $j \in I(Z)$ such that $a_{j}(Z) \neq a_{j+1}(Z)$. By replacing $Z$ by some iterate (if necessary) we can assume that $j=0$. By Lemma 7.3(1), (4), it follows that $a_{0}(Z) \neq 1$ and $a_{1}(Z) \neq 3$. Therefore there are two possibilities:

- $a_{0}(Z)=2$ and $a_{1}(Z)=1\left(\right.$ since $a_{0}(Z) \neq a_{1}(Z)$, from Lemma 7.3(2) we get $a_{1}(Z)=1$ ).

- $a_{0}(Z)=3$ and $a_{1}(Z)=1$ or 2 (observe that $a_{0}(Z) \neq a_{1}(Z)$ and $\left.a_{i}(Z) \neq 4\right)$.

In the first case, by Lemma 7.3(1), $a_{j}(Z)=1$ for all $j \geq 1$. In the second cases, by Lemma 7.3(1), (2), $a_{j}(Z)=1$ or 2 for every $j \geq 1$. In both cases we get $a_{j}(Z) \neq a_{0}(Z)$ for all $j \geq 1$. As in the proof of Corollary 7.5 this implies that the point $Z$ is wandering, which is a contradiction.

7.2. Hyperbolicity of the homoclinic classes of $P_{t}$ and $Q_{t}$ for $t$ in $L_{k}$. In the last section we proved that for any $t \in L_{k}$ every point of $W^{\mathrm{s}}\left(P_{t}\right) \cap W^{\mathrm{u}}\left(Q_{t}\right)$ is wandering and the diffeomorphism $f_{t}$ has no cycles associated to $P_{t}$ and $Q_{t}$ (Lemma 7.8). Moreover, the sequence of positions of any nonwandering point $Z$ of $\mathcal{V}$, different from $P_{t}$ and $Q_{t}$, is constant and equal to 1, 2 or 3 (Lemma 7.10). The next step is to characterize the points having a sequence of positions equal to 1 or 3 : every $Z \in \Omega\left(f_{t}\right)^{\prime}$ with a constant sequence of positions equal to 1 or 3 belongs either to the homoclinic class of $P_{t}$ (if the sequence consists of 1's) or to the homoclinic class of $Q_{t}$ (otherwise) (see Proposition 7.11). The converse of this assertion was stated in Corollary 7.7. Thereafter we prove that the homoclinic classes of $P_{t}$ and $Q_{t}$ are hyperbolic basic sets. In Section 8 we will consider the case where the sequence of positions is constant and equal to 2 . 
The next two results are similar to steps (E), (F) and (G) of $\left[\mathrm{DR}_{2}\right.$, Section 4.2]. Therefore we just sketch the main ideas involved in their proofs.

For each $i \in\{1,2,3\}$ let

$$
H_{i}(t)=\left\{Z \in \Omega\left(f_{t}\right) \cap \mathcal{V}: a_{j}(Z)=i \text { for every } j \in I(Z)\right\} .
$$

Proposition 7.11. One has

$$
\left\{P_{t}\right\} \cup H_{1}(t)=\overline{H\left(P_{t}\right)} \quad \text { and } \quad\left\{Q_{t}\right\} \cup H_{3}(t)=\overline{H\left(Q_{t}\right)} .
$$

Proof. By Corollary 7.7 it remains to prove the inclusions $H_{1}(t) \subset \overline{H\left(P_{t}\right)}$ and $H_{3}(t) \subset \overline{H\left(Q_{t}\right)}$. Let us prove this fact for $H_{3}(t)$; the other assertion follows similarly by considering backward orbits. We need the following lemma.

Lemma 7.12. Consider $Z \in H_{3}(t)$ and any $(n-r)$-dimensional disk $D$ containing $Z$ in its interior and transverse to the foliation $\mathcal{F}^{\mathrm{s}}$. There exists a sequence of disks in $W^{\mathrm{s}}\left(Q_{t}\right)$, say $\left(H_{n}\right)_{n \in \mathbb{N}}$, such that:

- every $H_{n}$ intersects $D$ transversely in a point $Z_{n}$,

- the angles of intersection between $D$ and $H_{n}$ at $Z_{n}$ are uniformly bounded from below, and

- the sequence $\left(Z_{n}\right)_{n \in \mathbb{N}}$ converges to $Z$.

Proof. For simplicity assume that $D$ is foliated by $(n-r-1)$-disks contained in leaves of $\mathcal{F}^{\mathrm{u}}$. First observe that if $I(Z)$ is bounded from above then, by Lemma 7.1, $Z \in W^{\mathrm{s}}\left(Q_{t}\right) \cup W^{\mathrm{s}}\left(P_{t}\right)$. Moreover, by Remark 7.9, if $Z \in W^{\mathrm{s}}(P) \cap \Omega\left(f_{t}\right)^{\prime}$ then $a_{j}(Z)=1$ for all $j \in I(Z)$. Thus, since $a_{j}(Z)=3$ for all $j$, we have $Z \in W^{\mathrm{s}}\left(Q_{t}\right)$. Now the result is immediate: we can take $Z_{n}=Z$ for all $n$ with the sequence $H_{n}$ being disks in $W^{\mathrm{s}}\left(Q_{t}\right)$ containing $Z_{n}$.

If $I(Z)$ is not bounded from above then, due to geometry of the cycle and the fact that one has expansion in the u-direction (parallel to $\mathcal{F}^{\mathrm{u}}$ ), there is a first $j \in I(Z)$ such that the connected component of $f_{t}^{r_{j}}(D) \cap \mathcal{V}_{1}$ containing $Z_{r_{j}}$ intersects $W^{\mathrm{s}}\left(P_{t}\right)$ along a curve $\alpha_{0}$ which is transverse to the stable foliation. Since $a_{j}(Z)=3$, by shrinking the size of $D$ in the central direction (if necessary), we can assume that $\alpha_{0}$ is contained in $\mathcal{I}_{3}$.

Given a curve $\alpha$ in $W_{\text {loc }}^{\text {cs }}\left(Q_{t}\right) \cap \mathcal{U}_{Q}\left(\right.$ see $(1.2)$ for the definition of $\left.W_{\text {loc }}^{\text {cs }}\left(Q_{t}\right)\right)$ denote by $\alpha^{\mathrm{c}}$ the set of the points $X^{\mathrm{c}}$ where $X \in \alpha$ (i.e., $\alpha^{\mathrm{c}}$ is the projection of $\alpha$ in $\gamma$ along the leaves of $\mathcal{F}^{\mathrm{s}}$ ). With this notation, the previous arguments imply that

$$
\alpha_{0}^{\mathrm{c}} \subset I_{3} .
$$

Denote by $D_{0}$ the component of $f_{t}^{r_{j}}(D) \cap \mathcal{V}_{1}$ containing $\alpha_{0}$ and write $W=W_{0}=Z_{r_{j}}$. Observe that $a_{j}(W)=3$ for all $j \geq 0$. We now let $h_{j}=h_{j}(W)=r_{j+1}(W)-r_{j}(W)=n\left(W_{r_{j}}\right)+n_{k}+N_{2}+m\left(W_{r_{j}}\right)+\left(-m_{k}\right)+N_{1}$. Recall that $n\left(W_{r_{j}}\right)+n_{k}$ and $m\left(W_{r_{j}}\right)+\left(-m_{k}\right)$ are the numbers of consecutive iterates of the orbit of $W_{r_{j}}$ in $\mathcal{U}_{Q}$ and in $\mathcal{U}_{P}$, respectively. Since $a_{j}(W)=3$, by Proposition $6.7(\mathrm{f}), m\left(W_{r_{j}}\right)=0$ or 1 . 
Let $D_{1}$ be the connected component of $f_{t}^{h_{1}}\left(D_{0}\right) \cap \mathcal{V}_{1}$ containing $W_{r_{1}}$ and

$$
\alpha_{1}=D_{1} \cap W_{\text {loc }}^{\text {cs }}\left(Q_{t}\right) \subset D_{1} \cap W^{\mathrm{s}}\left(P_{t}\right) .
$$

Write $W_{r_{1}}^{\mathrm{cs}}=\left(w_{r_{1}}^{\mathrm{s}}, w_{r_{1}}^{\mathrm{c}}, 0^{\mathrm{u}}\right)$ and observe that (by construction and $\left.(\mathrm{F} 1)\right)$ this point belongs to $\alpha_{1}$. Also observe that the curve $\alpha_{1}$ is transverse to the stable foliation. Then

$$
\alpha_{1}^{\mathrm{c}}=G_{n\left(W_{r_{1}}\right), m\left(W_{r_{1}}\right)}^{t}\left(\alpha_{0}^{\mathrm{c}}\right) .
$$

From (7.3) and Proposition 6.7(f) we get

$$
\left|\alpha_{1}^{\mathrm{c}}\right|>\varrho\left|\alpha_{0}^{\mathrm{c}}\right|, \quad \varrho>1
$$

We now argue inductively by considering disks $D_{i+1}$ defined as the connected components of $f^{h_{i+1}}\left(D_{i}\right) \cap \mathcal{V}$ containing $W_{r_{i+1}}$, curves $\alpha_{i+1}$ defined as the intersection between $D_{i+1}$ and $W_{\text {loc }}^{\text {cs }}\left(Q_{t}\right)$, and points $W_{r_{i}}^{\text {cs }}=$ $\left(w_{r_{i}}^{\mathrm{s}}, w_{r_{i}}^{\mathrm{c}}, 0^{\mathrm{u}}\right) \in \alpha_{i}$.

To estimate the lengths of the curves $\alpha_{i+1}$ we need to pay special attention to the previous construction (it is not true, in general, that $\left|\alpha_{i+1}^{\mathrm{c}}\right|>$ $\varrho\left|\alpha_{i}^{\mathrm{c}}\right|$ as in the case $i=0$ ). For that, for each $i \geq 0$, we consider the curves $\alpha_{i}^{+}$and $\alpha_{i}^{-}$defined as the closures of the connected components of $\alpha_{i} \backslash W_{r_{i}}^{\text {cs }}$ to the right and to the left of $W_{r_{i}}^{\text {cs }}$, respectively. Observe that

$$
\left(\alpha_{i+1}^{ \pm}\right)^{\mathrm{c}}=G_{n\left(W_{r_{i}}\right), m\left(W_{r_{i}}\right)}^{t}\left(\left(\alpha_{i}^{ \pm}\right)^{\mathrm{c}}\right) .
$$

Therefore, the calculation above for $i=0$ also shows that if $\left(\alpha_{i}^{ \pm}\right)^{\mathrm{c}}$ is contained in $I_{3}$ then

$$
\left|\left(\alpha_{i+1}^{ \pm}\right)^{\mathrm{c}}\right| \geq \varrho\left|\left(\alpha_{i}^{ \pm}\right)^{\mathrm{c}}\right| .
$$

First consider the curves $\alpha_{i}^{+}$. As above, if $\left(\alpha_{j}^{+}\right)^{\mathrm{c}}$ is contained in $I_{3}$ for all $j=0,1, \ldots, i$, then

$$
\left|\left(\alpha_{i+1}^{+}\right)^{\mathrm{c}}\right|>\varrho\left|\left(\alpha_{i}^{+}\right)^{\mathrm{c}}\right|>\varrho^{i+1}\left|\left(\alpha_{0}^{+}\right)^{\mathrm{c}}\right|, \quad \varrho>1 .
$$

As the maps $G_{n, m}^{t}$ preserve orientation, there is a (first) $k^{+}$such that $\left(\alpha_{k^{+}}^{+}\right)^{\mathrm{c}}$ intersects the right extreme of $I_{3}$. Then, using conditions (E1) and (E4), we find that $\left(\alpha_{i}^{+}\right)^{\mathrm{c}}$ intersects the right extreme of $I_{3}$ for all $i \geq k^{+}$.

Arguing analogously with the curves $\alpha_{j}^{-}$we get $k^{-}$such that $\left(\alpha_{i}^{-}\right)^{\mathrm{c}}$ intersects the left extreme of $I_{3}$ for all $i \geq k^{-}$. Taking $k=\max \left\{k^{+}, k^{-}\right\}$we see that $\alpha_{k}^{\mathrm{c}}$ contains $I_{3}$.

We now need the following fact.

Claim 7.13. Given $t \in L_{k}$ with $k$ large, consider the $(n-r)$-disk $H$ defined by

$$
H=\bigcup_{X \in \alpha} \mathcal{C C}\left(F^{\mathrm{u}}(X) \cap \mathcal{V}_{1}, X\right)
$$

where $\alpha$ is a curve in $W_{\mathrm{loc}}^{\mathrm{cs}}(Q)$ transverse to $\mathcal{F}^{\mathrm{s}}$ such that the interior of $\alpha^{\mathrm{c}}$ contains $I_{3}$. Then $W^{\mathrm{s}}\left(Q_{t}\right)$ intersects $H$ transversely. 
Proof. Observe that the condition $t \in L_{k}$ implies that the homoclinic point associated to $Q_{t}$ given by $Z_{t}=f_{t}^{-N_{2}+m_{k}}\left(Y_{t}\right)\left(Y_{t}\right.$ as in Section 1.3) is such that $Z_{t}^{\mathrm{c}}$ belongs to the interior of $J_{3}$. Observe now that

$$
G=\mathcal{C C}\left(W^{\mathrm{s}}\left(Q_{t}\right) \cap \mathcal{U}_{Q}, f_{t}^{-N_{1}}\left(Z_{t}\right)\right)=\left\{\left(x^{\mathrm{s}},\left(f_{t}^{-N_{1}}\left(z_{t}\right)\right)^{\mathrm{c}}, \widehat{z}_{t}^{\mathrm{u}}\right)\right\},
$$

where $\left(x^{\mathrm{s}}, 0^{\mathrm{c}}, 0^{\mathrm{u}}\right) \in \mathcal{U}_{Q}$ and $\widehat{z}_{t}^{\mathrm{u}} \in \mathbb{R}^{\mathrm{u}}$. Since $z_{t}^{\mathrm{c}} \in J_{3}$ we infer that $\left(f_{t}^{-N_{1}}\left(z_{t}\right)\right)^{\mathrm{c}}$ belongs to the interior of $I_{3}$. Thus $G$ intersects $H$ (transversely), ending the proof of the claim.

Using the claim we deduce that $f_{t}^{r_{k}}\left(D_{0}\right)$ intersects $W^{\mathrm{s}}\left(Q_{t}\right)$ transversely. Moreover, due to the geometry of the cycle, the angle of this intersection is bounded from below.

Finally, to get the sequence $Z_{n}$ it is enough to consider a nested sequence of disks $D_{n}$ contained in $D$ and containing $Z$ in their interiors and argue as before.

End of the proof of Proposition 7.11 $\left(H_{3}(t) \subset \overline{H\left(Q_{t}\right)}\right)$. If $I(Z)$ is lower bounded then, by Lemma 7.1, $Z$ belongs to $W^{\mathrm{u}}\left(Q_{t}\right) \cup W^{\mathrm{u}}\left(P_{t}\right)$, and, as $Z \in H_{3}(t)$, by Remark 7.9 , one has $Z \in W^{\mathrm{u}}\left(Q_{t}\right)$. Thus we can take the disk $D$ in Lemma 7.12 contained in $W^{\mathrm{u}}\left(Q_{t}\right)$ and containing $Z$ in its interior. Now applying Lemma 7.12 to the disk $D$ we get a sequence of points $Z_{n}$ such that

$$
Z_{n} \in D \pitchfork W^{\mathrm{s}}\left(Q_{t}\right) \subset W^{\mathrm{u}}\left(Q_{t}\right) \pitchfork W^{\mathrm{s}}\left(Q_{t}\right) \subset H\left(Q_{t}\right), \quad Z_{n} \rightarrow Z .
$$

Thus $Z \in \overline{H\left(Q_{t}\right)}$.

If $I(Z)$ is not lower bounded then we consider a nested sequence of disks, say $C_{n}$, contained in $F^{\mathrm{s}}(Z)$ and containing $Z$ in their interiors with $\bigcap C_{n}=\{Z\}$. For each $C_{n}$ there exists $-j_{n} \in I(Z)$ such that $f_{t}^{-j_{n}}\left(C_{n}\right)$ intersects $W^{\mathrm{u}}\left(Q_{t}\right)$ transversely at some point $Y_{n}$. Taking small $(n-r)$-disks in $W^{\mathrm{u}}\left(Q_{t}\right)$ around the points $Y_{n}$ and considering their images under $f_{t}^{j_{n}}$ one gets a sequence of disks in $W^{\mathrm{u}}\left(Q_{t}\right)$, say $D_{n}$, converging to a disk $D$ which is transverse to the stable foliation.

By Lemma $7.12, W^{\mathrm{s}}(Q)$ intersects $D$ transversely in a sequence of points $Z_{n}$ converging to $Z$. Due to the control on the angle of intersection, to each $Z_{n} \in W^{\mathrm{s}}\left(Q_{t}\right) \pitchfork D$ there corresponds a $\bar{Z}_{n} \in D_{n} \pitchfork W^{\mathrm{s}}\left(Q_{t}\right)$, and $\left(\bar{Z}_{n}\right)_{n}$ converges to $Z$. Since, by construction, each $\bar{Z}_{n} \in H\left(Q_{t}\right)$, this ends the proof of the proposition.

Proposition 7.14. Let $t \in L_{k}$ with $k$ sufficiently large. The homoclinic classes associated to $P_{t}$ and $Q_{t}$ are hyperbolic basic sets with indices $r+1$ and $r$, respectively. Moreover, there are no cycles related to these sets.

Proof. By definition, $\overline{H\left(P_{t}\right)}$ and $\overline{H\left(Q_{t}\right)}$ are transitive sets. To see, for example, that the homoclinic class of $P_{t}$ is locally maximal consider the set

$$
\mathcal{W}_{1}=\mathcal{V} \backslash\left(\mathcal{I}_{2} \cup \mathcal{I}_{3} \cup \mathcal{I}_{4} \cup \mathcal{V}_{1, t}\right) \text {, }
$$


where

$$
\mathcal{V}_{1, t}=\left\{Z \in \mathcal{U}_{Q}: 0 \leq z^{\mathrm{c}}<\beta(t)^{-n_{k}-1}\left(1-\varepsilon_{1}\right) a\right\},
$$

the sets $\mathcal{I}_{i}$ are defined in Section 2.1 and $I_{1}=\left[\left(1-\varepsilon_{1}\right) a, a\right]$. We claim that

$$
\overline{H\left(P_{t}\right)}=\bigcap_{n \in \mathbb{Z}} f_{t}^{n}\left(\mathcal{W}_{1}\right) .
$$

This can be seen as follows. Suppose that $Z \in \overline{H\left(P_{t}\right)}, Z \neq P_{t}$. Then considering some iterate of $Z$ we can assume that $Z \in \mathcal{V}_{1}$. By Corollary 7.7, $a_{i}(Z)=1$ for all $i \in I(Z)$, thus $Z_{r_{i}} \in \mathcal{I}_{1}$ for all $i \in I(Z)$. So what is left is to see that if $Z_{l} \in \mathcal{U}_{Q}$ then $Z_{l} \notin \mathcal{V}_{1, t}$. Since such a $Z_{l}$ is a backward iterate of some $Z_{r_{j}}$ with $Z_{l}, Z_{l+1}, \ldots, Z_{r_{j}} \in \mathcal{U}_{Q}$, the claim is equivalent to proving that $n\left(Z_{r_{j-1}}\right)=0$ or 1 , where

$$
r_{j}-r_{j-1}=n_{k}+n\left(Z_{r_{j-1}}\right)+N_{2}-m_{k}+m\left(Z_{r_{j-1}}\right)+N_{1} .
$$

But this is a consequence of Proposition 6.7(e). So $\overline{H\left(P_{t}\right)} \subset \bigcap_{i \in \mathbb{Z}} f_{t}^{i}\left(\mathcal{W}_{1}\right)$.

To prove the converse observe that, by definition,

$$
\bigcap_{i \in \mathbb{Z}} f_{t}^{i}\left(\mathcal{W}_{1}\right) \subset H_{1}(t) \cup\left\{P_{t}\right\}
$$

Thus, by Proposition 7.11, it follows that $\bigcap_{i \in \mathbb{Z}} f_{t}^{i}\left(\mathcal{W}_{1}\right) \subset \overline{H\left(P_{t}\right)}$.

To prove that $\overline{H\left(P_{t}\right)}$ is hyperbolic of index $r+1$ first observe that the derivative of $f_{t}$ contracts (resp. expands) uniformly the vectors parallel to the stable direction (tangent to $\mathcal{F}^{\mathrm{s}}$ ) (resp. the unstable direction). Thus, being hyperbolic, this set must have index either $r$ or $r+1$. To see that the index is $r+1$ we analyze the action of the derivative in the central direction.

As above, if $Z \in \overline{H\left(P_{t}\right)} \cap \mathcal{V}_{1}$ and it has a first return to $\mathcal{V}_{1}$, then by Proposition $6.7(\mathrm{e})$, this is a uniformly contracting return in the central direction. If the first return of $Z$ is not defined then $Z_{i}$ belongs to $W_{\mathrm{loc}}^{\mathrm{s}}\left(P_{t}\right)$ for all $i \geq N_{1}$ and it is clear that we have a contraction in the central direction.

Now the fact that $\overline{H\left(P_{t}\right)}$ is hyperbolic of index $r+1$ follows from the standard cone field construction.

In a similar way we find that $\overline{H\left(Q_{t}\right)}$ is a hyperbolic basic set of index $r$.

Finally, to prove that there are no cycles associated to $\overline{H\left(P_{t}\right)}$ and $\overline{H\left(Q_{t}\right)}$ just observe that if $Z \in W^{\mathrm{s}}\left(\overline{H\left(Q_{t}\right)}\right) \cap W^{\mathrm{u}}\left(\overline{H\left(P_{t}\right)}\right)$ then $Z \in \Omega\left(f_{t}\right) \cap \mathcal{V}$ and by Proposition 7.11 there are $j, k \in I(Z)$ such that $a_{j}(Z)=1$ and $a_{k}(Z)=3$, which contradicts Lemma 7.10.

7.3. Proof of the results for arcs close to the model family. We end this section by pointing out that the previous results (i.e., that the homoclinic classes of $P_{t}$ and $Q_{t}$ are hyperbolic basic sets for a set of parameters with positive relative density at $t=0$ ) remain true for a neighbourhood of the model arc $\left(f_{t}\right)_{t \in\left[-t_{0}, t_{0}\right]}$. 
In fact, observe that to obtain the result above we have only used conditions $(\mathrm{C} 1)-(\mathrm{C} 7),(\mathrm{E} 1)-(\mathrm{E} 8)$ and $(\mathrm{F} 1)$ and (F2). Clearly, conditions (C1)$(\mathrm{C} 7)$ and $(\mathrm{E} 1)-(\mathrm{E} 8)$ are open conditions (just replace $(\mathrm{C} 2)$ by a finite number of nonresonance conditions and (C6) by the transversality condition between $\gamma$ and the strong stable and unstable foliations of $W^{\mathrm{s}}(P)$ and $W^{\mathrm{u}}(Q)$, as explained in the comments after these conditions) and $\left\{M_{1}, M_{2}\right\}$ is also a filtration for diffeomorphisms close to $f=f_{0}$.

Of course, (F1) and (F2) are not open conditions: in general the foliations are not invariant along the cycle. However, they are almost-invariant in the sense that the returns of the leaves of the strong stable and of the strong unstable foliations are curves $C^{1}-t^{1+\eta}$-close to the initial leaves, for some $\eta>0$. This is sufficient for our purpose; for details see [DR 3 , Section 6.1].

Also in general the projections associated to the foliations are not isometries (see (F2)) but the general case is handled as in $\left[\mathrm{DR}_{3}\right.$, Section 6.2].

\section{SECONDARY CYCLES}

In view of the results in the previous section, to prove the theorem (for the model family) it remains to consider points in $\Omega\left(f_{t}\right) \cap \mathcal{V}, t \in L_{k}$ for sufficiently large $k$ (that we consider fixed in what follows), whose sequences of positions are constant and equal to 2 , i.e., $Z \in H_{2}(t)$.

Let $Z \in \Omega_{t} \cap \mathcal{V}_{1}$ and assume that $a_{j}(Z)=2$ for every $j \in I(Z)$. We claim that in this case $I(Z)=\mathbb{Z}$. To see this, observe that, for instance, if $I(Z)$ is upper bounded then, by Lemma $7.1, Z \in W^{\mathrm{s}}\left(P_{t}\right) \cup W^{\mathrm{s}}\left(Q_{t}\right)$. Thus, by Remark $7.9, a_{j}(Z)=1$ if $Z \in W^{\mathrm{s}}\left(P_{t}\right)$ and $a_{j}(Z)=3$ if $Z \in W^{\mathrm{s}}\left(Q_{t}\right)$ for all $j \in I(Z)$, which is a contradiction.

8.1. Central dynamics of points in $H_{2}(t)$. Recall that there are two kinds of returns for points $Z \in H_{2}(t)$ to $\mathcal{V}_{1}:(1,0)$ and $(0,1)$-returns. That is, in both cases the number $r_{1}=r_{1}(Z)$ is the same,

$$
r_{1}=n_{k}+N_{2}+\left(-m_{k}\right)+N_{1}+1 \text {. }
$$

However, for these two types of returns the restrictions of $f_{t}^{r_{1}}$ to the central direction are different (given by $G_{1,0}^{t}$ and $G_{0,1}^{t}$ ).

Lemma 8.1. Let $t \in L_{k}$. Then the diffeomorphism $f_{t}$ has two hyperbolic periodic points in $\mathcal{V}_{1}$, denoted by $A_{t}$ and $R_{t}$, of period

$$
\pi_{k}=n_{k}+N_{2}+\left(-m_{k}\right)+N_{1}+1
$$

and of indices $r+1$ and $r$, respectively. Moreover, $A_{t}^{\mathrm{c}}=\widetilde{A}_{t}$ and $R_{t}^{\mathrm{c}}=\widetilde{R}_{t}$.

Proof. By Proposition 6.1, the map $G_{0,1}^{t}$ has two fixed points $\widetilde{A}_{t}$ and $\widetilde{R}_{t}$. Observe also that $G_{0,1}^{t}$ corresponds to the central coordinates of returns under $f_{t}^{\pi_{k}}$. The result now follows by observing that the $\pi_{k}$-return of the 
diffeomorphism $f_{t}$ to $\mathcal{V}_{1}$ contracts uniformly the vectors tangent to $\mathcal{F}^{\mathrm{s}}$ and expands uniformly the vectors tangent to $\mathcal{F}^{\mathrm{u}}$.

8.2. Heterodimensional cycles associated to $A_{t}$ and $R_{t}$. In the next lemma we prove that there is a cycle associated to $A_{c_{k}}$ and $R_{c_{k}}$, where $c_{k} \in L_{k}$ is as in Lemma 6.4.

Lemma 8.2. For every $k$ sufficiently large the diffeomorphism $f_{c_{k}}$ has a cycle associated to the periodic points $A_{c_{k}}$ and $R_{c_{k}}, c_{k} \in L_{k}$. Moreover, the $\operatorname{arc}\left(f_{t}\right)_{t \in\left[a_{k}, b_{k}\right]}$ unfolds generically such a cycle.

Proof. We begin by observing that, for $t$ in $L_{k}$ and for the one-dimensional model $G_{0,1}^{t}$, we have

$$
\left(A_{t}^{\mathrm{c}}, R_{t}^{\mathrm{c}}\right)=\left(\widetilde{A}_{t}, \widetilde{R}_{t}\right) \subseteq W^{\mathrm{s}}\left(A_{t}^{\mathrm{c}}, G_{0,1}^{t}\right) \cap W^{\mathrm{u}}\left(R_{t}^{\mathrm{c}}, G_{0,1}^{t}\right) .
$$

By (F1), $A_{t}^{\mathrm{s}}=R_{t}^{\mathrm{s}}$ and $A_{t}^{\mathrm{u}}=R_{t}^{\mathrm{u}}$. Thus $W^{\mathrm{s}}\left(A_{t}, f_{t}\right)$ and $W^{\mathrm{u}}\left(R_{t}, f_{t}\right)$ are transverse along the orbit of the $f_{t}^{\pi_{k}}$-invariant $\left(\pi_{k}\right.$-periodic) curve

$$
\Upsilon_{t}=\left\{\left(A_{t}^{\mathrm{s}}, z^{\mathrm{c}}, A_{t}^{\mathrm{u}}\right): z^{\mathrm{c}} \in\left(A_{t}^{\mathrm{c}}, R_{t}^{\mathrm{c}}\right)\right\} .
$$

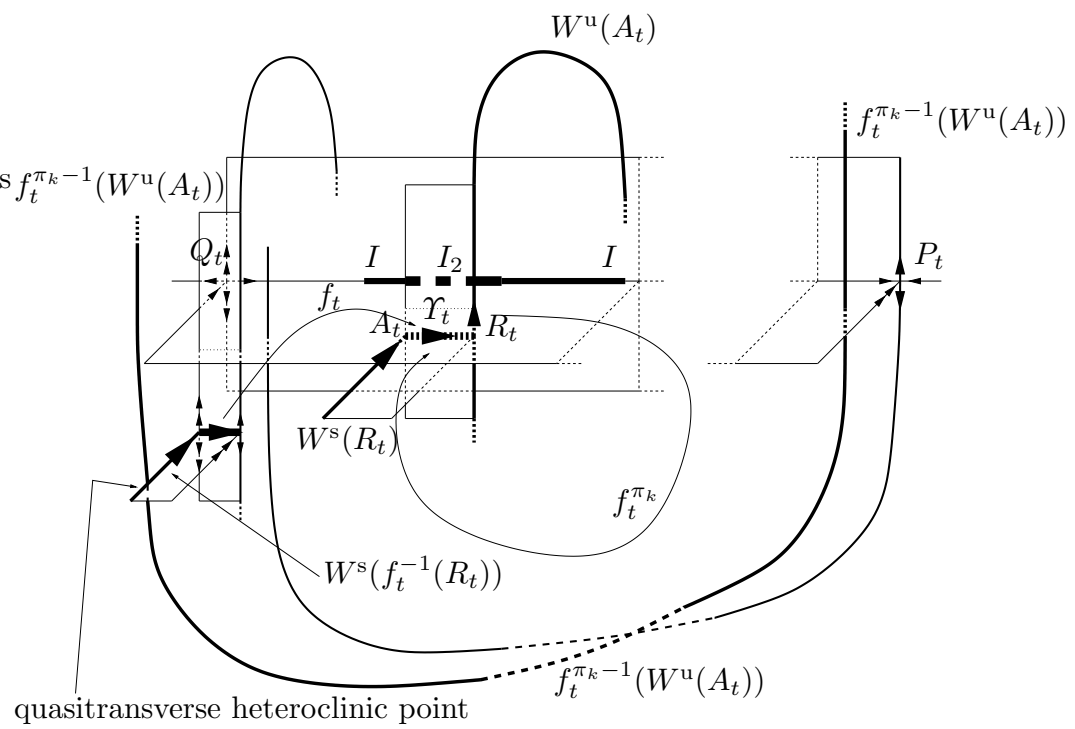

Fig. 7. The heterodimensional cycle at $c_{k}$

Observe that the numbers of iterates of the orbit of this periodic curve in $\mathcal{U}_{P}$ and $\mathcal{U}_{Q}$ are $-m_{k}+1$ and $n_{k}$, respectively, and that $n_{k} \rightarrow \infty$ and $-m_{k} \rightarrow \infty$ as $k \rightarrow \infty$. Now, using the expansion (resp. contraction) of the $\pi_{k}$-returns in the unstable (resp. stable) direction, it is not hard to check that $\Upsilon_{t}$ is close to the segment $\left\{0^{\mathrm{s}}\right\} \times(\widetilde{A}, \widetilde{R}) \times\left\{0^{\mathrm{u}}\right\}$. 
By (F1) the local manifolds of $A_{t}$ and $R_{t}$ are given by

$$
\begin{aligned}
& W_{\mathrm{loc}}^{\mathrm{u}}\left(A_{t}\right)=\left\{\left(A_{t}^{\mathrm{s}}, A_{t}^{\mathrm{c}}, x^{\mathrm{u}}\right):\left(0^{\mathrm{s}}, 0^{\mathrm{c}}, x^{\mathrm{u}}\right) \in \mathcal{U}_{Q}\right\}, \\
& W_{\mathrm{loc}}^{\mathrm{s}}\left(R_{t}\right)=\left\{\left(x^{\mathrm{s}}, R_{t}^{\mathrm{c}}, R_{t}^{\mathrm{u}}\right):\left(x^{\mathrm{s}}, 0^{\mathrm{c}}, 0^{\mathrm{u}}\right) \in \mathcal{U}_{Q}\right\} .
\end{aligned}
$$

Observe that there is a disk $\widetilde{D}_{t}^{\mathrm{u}}$ contained in $W_{\text {loc }}^{\mathrm{u}}\left(A_{t}\right)$ of points having a return of type $(0,0)$. By construction, the image of $\widetilde{D}_{t}^{\mathrm{u}}$ under $f_{t}^{\pi_{k}-1}$ contains a disk of the form

$$
D_{t}^{\mathrm{u}}=\left\{\left(w_{0}^{\mathrm{s}}, G_{0,0}^{t}\left(A_{t}^{\mathrm{c}}\right), x^{\mathrm{u}}\right):\left(0^{\mathrm{s}}, 0^{\mathrm{c}}, x^{\mathrm{u}}\right) \in \mathcal{U}_{Q}\right\} \subset f_{t}^{\pi_{k}-1}\left(\widetilde{D}_{t}^{\mathrm{u}}\right)
$$

for some $w_{0}^{\mathrm{s}}$. On the other hand, $f_{t}^{-1}\left(W_{\mathrm{loc}}^{\mathrm{s}}\left(R_{t}\right)\right)$ contains the set

$$
D_{t}^{\mathrm{s}}=\left\{\left(x^{\mathrm{s}}, \beta(t)^{-1} R_{t}^{\mathrm{c}}, w_{0}^{\mathrm{u}}\right):\left(x^{\mathrm{s}}, 0^{\mathrm{c}}, 0^{\mathrm{u}}\right) \in \mathcal{U}_{Q}\right\}
$$

for some $w_{0}^{\mathrm{u}}$. Thus to get an intersection between $D_{t}^{\mathrm{u}}$ and $D_{t}^{\mathrm{s}}$ (which will be necessarily quasi-transverse and will provide the cycle) it is enough to observe that

$$
\beta(t)^{-1} R_{t}^{\mathrm{c}}=G_{0,0}^{t}\left(A_{t}^{\mathrm{c}}\right), \quad t=c_{k} \in L_{k}
$$

(see Lemma 6.4).

It remains to see that the arc unfolds this cycle with positive velocity. But, as this velocity is equal to the derivative of $U_{k}(t)$ at $c_{k}$, this also follows from Lemma 6.4.

The next lemma together with Proposition 7.14 proves items (2) and (3) of the theorem and shows that $c_{k}$ is a first bifurcation value for $\left(f_{t}\right)_{t \in\left[-t_{0}, t_{0}\right]}$.

Lemma 8.3. Consider $\left.t \in] c_{k}, b_{k}\right]$. Then

$$
\Omega\left(f_{t}\right)^{\prime}=\overline{H\left(P_{t}\right)} \cup \overline{H\left(Q_{t}\right)} \cup\left\{\mathcal{O}\left(A_{t}\right), \mathcal{O}\left(R_{t}\right)\right\} .
$$

Proof. Fix any $\left.t \in] c_{k}, b_{k}\right]$ with $k$ large. In view of Corollary 7.5 and Propositions 7.11 and 7.14, it is enough to see that $H_{2}(t)=\left\{\mathcal{O}\left(A_{t}\right), \mathcal{O}\left(R_{t}\right)\right\}$. Clearly, $\left\{\mathcal{O}\left(A_{t}\right), \mathcal{O}\left(R_{t}\right)\right\} \subset H_{2}(t)$. The other inclusion is a direct consequence of Proposition 6.7(b).

Observe that our construction also gives the following:

$$
W^{\mathrm{s}}\left(A_{t}\right) \cap W^{\mathrm{u}}\left(R_{t}\right)=\bigcup_{i=0}^{\pi_{k}} f_{t}^{i}\left(\Upsilon_{t}\right), \quad \Upsilon_{t}=\left\{\left\{A_{t}^{\mathrm{s}}\right\} \times\left(A_{t}^{\mathrm{c}}, R_{t}^{\mathrm{c}}\right) \times\left\{A_{t}^{\mathrm{u}}\right\}\right\} .
$$

\section{NONHYPERBOLIC ROBUSTLY TRANSITIVE DYNAMICS}

9.1. The set $H_{2}(t)$ for $t \in\left[a_{k}, c_{k}[\right.$. In this section we prove item (4) of the theorem for the model family. This result follows immediately from:

Proposition 9.1. Let $t \in\left[a_{k}, c_{k}\left[\right.\right.$. Then $H_{2}(t)=\overline{H\left(A_{t}\right)}=\overline{H\left(R_{t}\right)}$. 
Observe that the cycles associated to $A_{c_{k}}$ and $R_{c_{k}}$ we consider here are exactly the ones considered in $\left[\mathrm{D}_{1}\right.$, Theorem 1$]$, where it is proved that the resulting nonwandering set is equal to the homoclinic classes of $P_{t}$ and $Q_{t}$. To prove these results one only needs to have a diffeomorphism $f_{c_{k}}$ having a restriction to the curve $\left[\widetilde{A}_{t}, \widetilde{R}_{t}\right]$ with fine distortion properties (as in our case). So we just sketch the proof of this proposition.

Proof of Proposition 9.1. Observe first that by the choice of the parameters, $\overline{H\left(R_{t}\right)} \subset H_{2}(t)$ and $\overline{H\left(A_{t}\right)} \subset H_{2}(t)$. Thus we need to prove

Proposition 9.2. Let $t \in\left[a_{k}, c_{k}[\right.$. Then

$$
H_{2}(t) \subset \overline{H\left(R_{t}\right)} \quad \text { and } \quad H_{2}(t) \subset \overline{H\left(A_{t}\right)} .
$$

Proof. To prove the first inclusion, we need the following two preparatory lemmas:

Lemma 9.3. Let $t \in\left[a_{k}, c_{k}\left[, x \in H_{2}(t)\right.\right.$ and $\Delta$ an $(n-r)$-dimensional rectangle containing $x$ foliated by segments of the strong unstable foliation $\mathcal{F}^{\mathrm{u}}$. Then $\Delta \pitchfork W^{\mathrm{s}}\left(R_{t}\right) \neq \emptyset$.

Proof. Using expansion in the unstable direction and the fact that, by definition, $a_{j}(x)=2$ for all $j$, we get $n>0$ such that $f_{t}^{n}(\Delta)$ contains a rectangle $\Delta^{\prime}$ that (in local coordinates) is of the form $\left\{x_{0}^{\mathrm{s}}\right\} \times U \times[-1,1]$, where $U$ is contained in $I_{2}$.

Recall now that each map $F_{n, m(\nu)+1}^{t}$ of the iterated function system $\mathcal{F}^{t}$ defined in Section 4.2 corresponds to some power of $f_{t}$. By an argument as in Section 7, Proposition 4.11 implies now that there is $k>0$ such that $f_{t}^{k}\left(\Delta^{\prime}\right) \pitchfork W^{\mathrm{s}}\left(R_{t}\right) \neq \emptyset$, ending the proof of the lemma.

Remark 9.4. As in Remark 7.4, Lemma 9.3 also holds if $x$ is a point whose forward orbit is contained in $\mathcal{V}$ and whose itinerary consists of 2's.

Lemma 9.5. Let $t \in\left[a_{k}, c_{k}\right.$ [ and $x \in H_{2}(t)$. Consider any small stable segment $\Upsilon$ containing $x$ in its interior. Then $\Upsilon \pitchfork W^{\mathrm{u}}\left(R_{t}\right) \neq \emptyset$.

Proof. Just observe that there is expansion in the stable direction for negative iterates, and that the backward iterates of $x$ are always to the left of $R_{t}$.

We are now ready to prove that $H_{2}(t) \subset \overline{H\left(R_{t}\right)}$. Given $x \in H_{2}(t)$ and $\varepsilon>0$ consider a stable segment $\Upsilon_{\varepsilon}$ of size $2 \varepsilon$ centred at $x$. By Lemma 9.5, there is $z \in \Upsilon_{\varepsilon} \pitchfork W^{\mathrm{u}}\left(R_{t}\right)$. So there is a rectangle $\Delta_{\varepsilon}$ of diameter less than $\varepsilon$ containing $z$ and contained in $W^{\mathrm{u}}\left(R_{t}\right)$. Since $z$ is in a stable segment of $x$ the forward orbit of $z$ is contained in $\mathcal{V}$ and its forward itinerary consists of 2's. By Remark 9.4 and Lemma 9.3, $\Delta_{\varepsilon} \cap H\left(R_{t}\right) \neq \emptyset$. This implies that there is a homoclinic point of $R_{t}$ at distance less than $2 \varepsilon$ from $x$. Since this 
construction holds for all $\varepsilon$ this implies that $x \in \overline{H\left(R_{t}\right)}$, ending the proof of $H_{2}(t) \subset \overline{H\left(R_{t}\right)}$.

Observe that the inclusion $H_{2}(t) \subset \overline{H\left(A_{t}\right)}$ follows analogously by using the iterated function system $\widetilde{\mathcal{F}}^{\mu}$ and Remark 4.13. The proof of Proposition 9.2 is now complete.

As we mentioned above, Proposition 9.1 follows immediately from Proposition 9.2.

9.2. Proof of item (4) of the theorem: the general case. Recall that a strongly partially hyperbolic set of a diffeomorphism $f$ is a compact $f$-invariant set $\Lambda$ such that there are a splitting of the tangent bundle over $\Lambda$ into three nontrivial $D f$-invariant subbundles, $T_{\Lambda}=E^{\mathrm{ss}} \oplus E^{\mathrm{c}} \oplus E^{\mathrm{uu}}$, and constants $K>0,0<\varrho<1$ and $j>0$ such that:

- $E^{\mathrm{ss}}$ and $E^{\mathrm{uu}}$ are uniformly hyperbolic bundles (contracting and expanding, respectively).

- Write $E^{\mathrm{cs}}=E^{\mathrm{ss}} \oplus E^{\mathrm{c}}$ and $E^{\mathrm{cu}}=E^{\mathrm{uu}} \oplus E^{\mathrm{c}}$. Then the splittings $E^{\mathrm{ss}} \oplus E^{\mathrm{cu}}$ and $E^{\mathrm{cs}} \oplus E^{\mathrm{uu}}$ are dominated, i.e., for every $x \in \Lambda$ one has

$$
\begin{aligned}
& \left\|\left.D_{x} f^{j}\right|_{E_{x}^{\text {ss }}}\right\| \cdot\left\|\left.D_{f^{j}(x)} f^{-j}\right|_{E_{f^{j}(x)}^{\mathrm{cu}}}\right\|<K \varrho^{j}, \\
& \left\|\left.D_{x} f^{-j}\right|_{E_{x}^{\mathrm{uu}}}\right\| \cdot\left\|\left.D_{f^{-j}(x)} f^{j}\right|_{E_{f^{-j}(x)}^{\mathrm{cs}}}\right\|<K \varrho^{j} .
\end{aligned}
$$

Observe that in our model the sets $\overline{H\left(P_{t}\right)}, \overline{H\left(Q_{t}\right)}, \overline{H\left(R_{t}\right)}$, and $\overline{H\left(A_{t}\right)}$ are strongly partially hyperbolic.

Consider an $\operatorname{arc}\left(h_{\mu}\right)_{\mu \in[-\tau, \tau]}, \tau>0$, of diffeomorphisms in $\mathbb{R}^{n}$ unfolding generically a heterodimensional cycle at $\mu=0$ associated to the hyperbolic fixed points $A_{0}=\left(0^{r}, \widetilde{A}, 0^{n-r-1}\right)$ and $R_{0}=\left(0^{r}, \widetilde{R}, 0^{n-r-1}\right)$ of indices $r+1$ and $r(\widetilde{A}$ and $\widetilde{R}$ are the points introduced in (E9)) satisfying conditions (C1)(C7) of Section 1 and the conditions on the unfolding of a heterodimensional cycle described at the beginning of Section 1.3. Moreover, the arc $\left(h_{\mu}\right)_{\mu \in[-\tau, \tau]}$ is constructed to satisfy the following conditions:

(M1) Geometry of the intersections: $W^{\mathrm{s}}\left(A_{0}\right) \cap W^{\mathrm{u}}\left(R_{0}\right)=W^{\mathrm{s}}\left(A_{0}\right) \pitchfork$ $W^{\mathrm{u}}\left(R_{0}\right)=\left\{0^{r}\right\} \times(\widetilde{A}, \widetilde{R}) \times\left\{0^{n-r-1}\right\}=\Upsilon_{0}$; in particular, the cycle is connected.

(M2) Central dynamics: For some small $\eta>0$ the restriction of $h_{\mu}$ to the curve $\left\{0^{r}\right\} \times[\widetilde{A}-\eta, \widetilde{R}+\eta] \times\left\{0^{n-r-1}\right\}$ is given by $\left(T_{0,1}^{\mu}\right)^{N_{3}}$ (recall (E9)). In particular, $\left\{0^{r}\right\} \times\left[\widetilde{A}_{\mu}, \widetilde{R}_{\mu}\right] \times\left\{0^{n-r-1}\right\}$ is contained in the transverse intersection between $W^{\mathrm{s}}\left(A_{\mu}\right)$ and $W^{\mathrm{u}}\left(R_{\mu}\right)$.

(M3) Transverse dynamics: The positive eigenvalues $\lambda_{A}$ and $\beta_{R}$ of the derivative of $T$ at $A$ and $R$ are such that $\lambda_{A}$ is the greatest contracting eigenvalue of $D h_{0}(A)$ (in modulus) and $\beta_{R}$ is the smallest 
expanding eigenvalue of $D h_{0}(R)$ (in modulus). Moreover, any eigenvalue $\sigma$ of $D h_{0}(A)$ (resp. $\left.D h_{0}(R)\right)$ different from $\lambda_{A}$ (resp. $\beta_{R}$ ) is such that $|\sigma| \neq\left|\lambda_{A}\right|$ (resp. $|\sigma| \neq\left|\beta_{R}\right|$ ).

(M4) The cycle is noncritical: By (M3) one can define the strong stable foliation of $W^{\mathrm{s}}\left(A_{0}\right)$ and the strong unstable foliation of $W^{\mathrm{u}}\left(R_{0}\right)$. We assume that the curve $\Upsilon_{0}$ is transverse to these foliations. Moreover, the cycle satisfies conditions (F1) and (F2) of Section 1.2.

(M5) Velocity of the unfolding of the cycle: The cycle is unfolded with positive velocity (recall Lemma 8.2).

Finally, the neighbourhood of the cycle and the constants $C, C^{\prime}$ and $r$ above depend only on the geometry of the cycle (conditions (M1) and (M4)), the dynamics of the restriction of $h_{0}$ to $\Upsilon_{0}$ (condition (M2)), and the velocity of the unfolding (M5). In other words, these constants do not depend on the transverse dynamics of the cycle once condition (M3) is satisfied.

For each $k$ large enough consider the interval $L_{k}$ and the reparametrization $\mu_{k}: L_{k} \rightarrow\left[-\mu_{0}, \mu_{0}\right]$ defined in Lemma 5.7 and its inverse $t_{k}(\mu)$. Now define the arc $\left(g_{k, \mu}\right)_{\mu \in\left[-\mu_{0} / 2, \mu_{0} / 2\right]}$ of diffeomorphisms by

$$
g_{k, \mu}=f_{t_{k}(\mu)}^{\pi_{k}},
$$

where $\pi_{k}=n_{k}+N_{2}-m_{k}+1+N_{1}$ (Lemma 8.1). By Lemma 8.2, this arc unfolds generically at $\theta_{k}=\mu_{k}\left(c_{k}\right)$ a cycle associated to the hyperbolic fixed points $A_{c_{k}}$ and $R_{c_{k}}$.

Lemma 9.6. For every large $k$ and small $\mu_{0}$ the arc $\left(g_{k, \mu}\right)_{\mu \in\left[-\mu_{0} / 2, \mu_{0} / 2\right]}$ is $\varepsilon-C^{1}$-close to some arc $\left(h_{\mu}\right)_{\mu \in\left[-\mu_{0} / 2, \mu_{0} / 2\right]}$ satisfying conditions (M1)-(M5).

Having in mind the comments we made in Section 7.3 and the construction in Section 9.1, it is now clear that we can argue exactly in the same way for arcs close to $\left(f_{t}\right)_{t \in\left[-t_{0}, t_{0}\right]}$ getting the same conclusion. Moreover, using the standard cone-field construction one concludes that the sets $\overline{H\left(P_{t}\right)}$, $\overline{H\left(Q_{t}\right)}, \overline{H\left(A_{t}\right)}$, and $\overline{H\left(R_{t}\right)}$ are strongly partially hyperbolic.

\section{References}

[BDP] C. Bonatti, L. J. Díaz, and E. R. Pujals, $A \mathcal{C}^{1}$-generic dichotomy for diffeomorphisms: Weak forms of hyperbolicity or of infinitely many sinks or sources, Ann. of Math., to appear.

[BDPR] C. Bonatti, L. J. Díaz, E. R. Pujals, and J. Rocha, Robustly transitive sets and heterodimensional cycles, Astérisque, to appear.

$\left[D_{1}\right] \quad$ L. J. Díaz, Robust nonhyperbolic dynamics and heterodimensional cycles, Ergodic Theory Dynam. Systems 15 (1995), 291-315.

$\left[\mathrm{D}_{2}\right] \quad-$, Persistence of cycles and nonhyperbolic dynamics at heteroclinic bifurcations, Nonlinearity 8 (1995), 693-713. 
[DPU] L. J. Díaz, E. R. Pujals, and R. Ures, Partial hyperbolicity and robust transitivity, Acta Math. 183 (2000), 1-43.

[DR $\mathrm{DR}_{1} \quad$ L. J. Díaz and J. Rocha, Nonconnected heterodimensional cycles: bifurcation and stability, Nonlinearity 5 (1992), 1315-1341.

$\left[\mathrm{DR}_{2}\right]-,-$, Large measure of hyperbolic dynamics when unfolding heteroclinic cycles, ibid. 10 (1997), 857-884.

[DR 3$] \quad-,-$, Partially hyperbolic and transitive dynamics generated by heteroclinic cycles, Ergodic Theory Dynam. Systems 21 (2001), 25-76.

[KKY] I. Kan, H. Koçak, and J. Y. Yorke, Antimonotonicity: Concurrent creation and annihilation of periodic orbits, Ann. of Math. 136 (1992), 219-252.

[MT] J. Milnor and W. P. Thurston, On iterated maps of the interval, in: Lecture Notes in Math. 1342, Springer, 1988, 465-563.

$\left[\mathrm{N}_{1}\right] \quad$ S. Newhouse, Hyperbolic limit sets, Trans. Amer. Math. Soc. 167 (1972), 125150 .

$\left[\mathrm{N}_{2}\right] \quad-$, The abundance of wild hyperbolic sets and nonsmooth stable set for diffeomorphisms, Publ. Math. I.H.E.S. 50 (1979), 101-151.

$\left[\mathrm{PT}_{1}\right] \quad$ J. Palis and F. Takens, Cycles and measure of the bifurcation set for two dimensional diffeomorphisms, Invent. Math. 92 (1985), 397-422.

$\left[\mathrm{PT}_{2}\right] \quad-,-$, Hyperbolicity and the creation of homoclinic orbits, Ann. of Math. 125 (1987), 337-374.

[PT $\left.\mathrm{PT}_{3}\right]-$ - - Hyperbolicity and Sensitive Chaotic Dynamics at Homoclinic Bifurcations, Cambridge Univ. Press, 1993.

Depto. Matemática PUC-Rio

Marquês de S. Vicente núm. 225

22453-900 Rio de Janeiro RJ, Brazil

E-mail: lodiaz@mat.puc-rio.br
Departamento de Matemática Pura

Universidade do Porto Rua do Campo Alegre núm. 687 4169-007 Porto, Portugal E-mail: jrocha@fc.up.pt

Received 18 April 2000;

in revised form 23 July 2001 